\title{
WestVirginiaUniversity
}

THE RESEARCH REPOSITORY @ WVU

Graduate Theses, Dissertations, and Problem Reports

1999

\section{Through-wafer interrogation of MEMS device motion}

Jeremy Michael Dawson

West Virginia University

Follow this and additional works at: https://researchrepository.wvu.edu/etd

\section{Recommended Citation}

Dawson, Jeremy Michael, "Through-wafer interrogation of MEMS device motion" (1999). Graduate Theses, Dissertations, and Problem Reports. 967.

https://researchrepository.wvu.edu/etd/967

This Thesis is protected by copyright and/or related rights. It has been brought to you by the The Research Repository @ WVU with permission from the rights-holder(s). You are free to use this Thesis in any way that is permitted by the copyright and related rights legislation that applies to your use. For other uses you must obtain permission from the rights-holder(s) directly, unless additional rights are indicated by a Creative Commons license in the record and/ or on the work itself. This Thesis has been accepted for inclusion in WVU Graduate Theses, Dissertations, and Problem Reports collection by an authorized administrator of The Research Repository @ WVU. For more information, please contact researchrepository@mail.wvu.edu. 


\title{
Through-Wafer Interrogation of MEMS Device Motion
}

\author{
Jeremy M. Dawson \\ B.S.E.E, West Virginia University \\ THESIS \\ Submitted to \\ the College of Engineering and Mineral Resources \\ at \\ WEST VIRGINIA UNIVERSITY \\ in partial fulfillment of the requirements for the degree of
}

Master of Science in Electrical Engineering

\author{
Examining Committee Members: \\ Dr. Larry A. Hornak (Committee Chair) \\ Dr. Parviz Famouri \\ Dr. Biswajit Das
}

Morgantown, West Virginia

1999

Keywords: Optical monitoring, Optical Metrology 


\title{
ABSTRACT \\ Through-Wafer Interrogation of MEMS Device Motion
}

\author{
Jeremy M. Dawson
}

\begin{abstract}
Microelectromechanical systems (MEMS) have been the focus of many research groups because of their wide variety of uses in sensing and actuation applications. A fundamental barrier facing designers of next generation MEMS is the inability to access accurate, real-time microstructure positional information to determine if the device is performing as expected. Previously explored optical and electrical methods of MEMS device monitoring are often only suitable for research environments, or are unable to produce clear and meaningful characterization of device motion. The most desirable MEMS monitoring method would be one that could be implemented at the device level, which would allow the monitoring system to be fabricated along with the device itself. This research explores a through-wafer method of optically monitoring and characterizing the motion of a lateral comb resonator fabricated using the Multi-User MEMS Process Service (MUMPS). Positional monitoring results obtained from a $1.3 \mu \mathrm{m}$ wavelength through-wafer optical probe are presented, as well as a method of device level implementation of the monitoring system.
\end{abstract}




\section{Acknowledgments}

I would like to thank my committee members, Dr. Larry Hornak, Dr. Parviz

Famouri, and Dr. Biswajit Das, especially Dr. Hornak, for all of the guidance and support they supplied throughout the duration of this work. I would also like to thank my colleagues Kolin Brown and Steve McGinnis, as well as other members of the MSRC and affiliated centers, for help, criticism, and forcing me to become a self-motivated research engineer. I would like to thank Dr. Halliburton, Kevin Stevens, and the Physics Department at WVU for allowing me to use their polishing equipment.

I would especially like to thank my family for their prayers and support during this work, and my roommates for putting up with my antisocial attitude during the final stages of thesis writing and experimentation.

Last, but not least, I would like to thank God for walking beside me during, not only this, but all of life's difficulties, both large and small. 


\section{Contents}

Abstract $\quad$ ii

Acknowledgements

List of Figures $\quad$ vii

List of Tables $\quad$ xii

1 Introduction 1

1.1 Microelectromechanical Systems 3

$\begin{array}{ll}\text { 1.1.1 Translational MEMS } & 3\end{array}$

1.1.2 Torsional/Vertical Motion MEMS 6

1.1.3 Gear/Rotary Motion MEMS 9

1.2 Optical Interconnect Technologies 11

$\begin{array}{ll}\text { 1.2.1 Optical Waveguides } & 12\end{array}$

1.2.2 Through-Wafer Interconnects 13

$\begin{array}{ll}\text { 1.2.3 Diffractive/Refractive Optics } & 14\end{array}$

$\begin{array}{ll}\text { 1.3 Optical MEMS Device Monitoring } & 15\end{array}$

2 Theory 18

$\begin{array}{ll}\text { 2.1 MEMS Motion } & 19\end{array}$

2.2 Multi-Layer Transmission 21

2.3 Optical Probe Beam Generation and Manipulation 24

2.4 Diffractive Optics/Total Internal Reflection Propagation 30 
2.4.1 On-Axis Fresnel Lenses $\quad 30$

2.4.2 Off-Axis Fresnel Lenses 31

2.4.3 TIR Glass Slab Propagation 33

3 MEMS Design $\quad 34$

3.1 Design and Analysis 36

3.2 Device Layout 49

3.3 Release Process $\quad 57$

3.4 Through-Wafer Utilization $\quad 57$

3.5 Backside Wafer Polishing 58

4 Through-Wafer Testing 59

4.1 Expected Transmission $\quad 59$

$\begin{array}{ll}\text { 4.2 Experimental Setup } & 62\end{array}$

4.3 Infrared Light Sources/Detectors $\quad 64$

$\begin{array}{ll}\text { 4.3.1 LED } & 64\end{array}$

$\begin{array}{ll}\text { 4.3.2 Laser Diode } & 65\end{array}$

4.3.3 Germanium Detector 66

$\begin{array}{ll}\text { 4.3.4 InGaAs Detector } & 67\end{array}$

$\begin{array}{ll}\text { 4.4 GRIN Lens Applications } & 67\end{array}$

$\begin{array}{ll}\text { 4.5 Image Capture Results } & 70\end{array}$

$\begin{array}{ll}\text { 4.6 Power Measurement } & 74\end{array}$

4.7 Optical Monitoring of Static Deflection 90

4.8 Optical Monitoring During MEMS Actuation 92

4.8.1 Spot Position Variation 93 
$\begin{array}{ll}\text { 4.8.2 Frequency Variation } & 95\end{array}$

$\begin{array}{ll}\text { 4.8.3 Voltage Variation } & 99\end{array}$

5 Loss Analysis and Conclusions $\quad 102$

5.1 Experimental Setup Loss Analysis 102

5.2 Loss Analysis of Proposed Integrated Through-Wafer

$\begin{array}{ll}\text { Optical Monitoring System } & 103\end{array}$

$\begin{array}{ll}\text { 5.3 Conclusions and Future Work } & 105\end{array}$

Appendices:

$\begin{array}{lll}\text { A MATLAB MEMS Analysis Programs } & 107\end{array}$

B Microelectronics Center of North Carolina (MCNC) Multi-User MEMS Processing Service (MUMPS) and Film Parameters 116

$\begin{array}{lr}\text { Bibliography } & 119\end{array}$

$\begin{array}{lr}\text { Signature Page } & 123\end{array}$ 


\section{List of Figures}

1.1 Lateral Comb Resonator Designed at WVU 4

1.2 Free-Space Microoptical Bench 5

$\begin{array}{ll}1.3 \text { Optical Switch } & 6\end{array}$

$\begin{array}{ll}1.4 \text { Deformable Micromirror } & 7\end{array}$

1.5 Digital Micromirror Display 99

$\begin{array}{ll}1.6 \text { The Sandia Microengine } & 10\end{array}$

1.7 Planar Device Monitoring $\quad 15$

1.8 Diffractive Optics Device Monitoring 15

1.9 Through-Wafer Device Monitoring 16

2.1 Lateral Comb Resonator with Folded Flexure Design 19

2.2 Plane Interface 22

2.3 Multiple Layers 23

2.4 Transmission of a Gaussian Beam Through a Thin Lens 25

2.5 GRIN Lens Focal Points 27

2.6 Double GRIN Lens Setup 28

2.7 Beam Radius at GRIN Lens 29

2.8 Fresnel Lens Cross-Sections 32

2.9 Off-Axis Lens Configuration 32 
2.10 TIR Slab Propagation 33

3.1 Phase I Stage Geometry $\quad 35$

3.2 Phase II stage Geometry $\quad 35$

3.3 Length of Flexure vs. Spring Constant - Geometry B 37

3.4 Length of Flexure vs. Displacement - Geometry B 38

3.5 Length of Flexure vs. Resonant Frequency - Geometry B 39

3.6 Voltage vs. Displacement - Geometry B 40

3.7 Length of Flexure vs. Displacement - Geometry A 42

3.8 Length of Flexure vs. Resonant Frequency - Geometry A 43

3.9 Voltage vs. Displacement - Geometry A 44

3.10 Flexure Length vs. Displacement - Geometry C 46

3.11 Length of Flexure vs. Resonant Frequency - Geometry C 47

3.12 Voltage vs. Displacement - Geometry C 48

3.13 Device Layout on $1 \mathrm{~cm} \times 1 \mathrm{~cm}$ Die $\quad 50$

$\begin{array}{ll}3.14 \text { Geometry A } & 51\end{array}$

3.15 Close-up of Monolithic Structure 52

3.16 Geometry B $\quad 53$

3.17 Geometry C $\quad 54$

3.18 Close-up of Reinforced Comb Fingers 55

3.19 Device Identification and Alignment Marks 56

$\begin{array}{ll}\text { 4.1 Device Layers } & 60\end{array}$

4.2 Reflectance as a Function of Nitride Thickness 61

4.3 Experimental Setup 63 
4.4 Representation of Beam Interaction with Device Stage 63

4.5 LED Pin Configuration $\quad 65$

4.6 Laser Diode Pin Configuration $\quad 66$

$\begin{array}{ll}4.7 \text { Object vs. Image Distances } & 68\end{array}$

$\begin{array}{ll}4.8 \text { Object Distance vs. Magnification } & 68\end{array}$

4.9 Object Distance vs. Spot Size 69

4.10 Object Distance vs. Beam Diameter at Lens 69

4.11 Spot Progression Under Device Stage (Phase II Device) 71

4.12 Spot Progression Under Flexures 72

4.13 Through-Wafer Spot Intensity Under Translation Stage 72

4.14 Through-Wafer Spot Intensity Under Translation Flexure 73

$\begin{array}{ll}\text { 4.15 Total Intensity as a Function of Distance } & 74\end{array}$

$\begin{array}{ll}\text { 4.16 Gaussian Beam } & 75\end{array}$

4.17 Device Transmission Function 76

$\begin{array}{ll}4.18 \text { Convolution (side view) } & 76\end{array}$

4.19 Contact Pad Width

$\begin{array}{ll}\text { 4.20 Contact Connection Strip Width } & 78\end{array}$

4.21 Through-Wafer Voltage Under Contact Pad (LD, $47 \mu \mathrm{m}$ detector fiber, double $\begin{array}{ll}\text { GRIN lenses) } & 79\end{array}$

4.22 Through-Wafer Power Under Contact Pad (LD, $47 \mu \mathrm{m}$ detector fiber, double GRIN lenses) $\quad 79$

4.23 Through-Wafer Voltage Under Stage (LD, $47 \mu \mathrm{m}$ detector fiber, double GRIN lenses)

4.24 Through-Wafer Power Under Stage (LD, $47 \mu \mathrm{m}$ detector fiber, double GRIN lenses) 
4.25 Through-Wafer Voltage Under Contact Pad (LD, $8 \mu \mathrm{m}$ detector fiber, double GRIN lenses)

4.26 Through-Wafer Power Under Contact Pad (LD, $8 \mu$ m detector fiber, double GRIN lenses)

4.27 Through-Wafer Voltage Under Stage (LD, $8 \mu \mathrm{m}$ detector fiber, double GRIN lenses)

4.28 Through-Wafer Power Under Stage (LD, $8 \mu \mathrm{m}$ detector fiber, double GRIN lenses)

4.29 Area of IR Scan

4.30 Through-Wafer Voltage (LED, $100 \mu \mathrm{m}$ detector fiber, single GRIN lens)

4.31 Through-Wafer Power (LED, $100 \mu \mathrm{m}$ detector fiber, single GRIN lens)

4.32 Through-Wafer Voltage (LD, $100 \mu \mathrm{m}$ detector fiber, double GRIN lenses)

4.33 Through-Wafer Power (LD, $100 \mu \mathrm{m}$ detector fiber, double GRIN lenses)

4.34 Through-Wafer Voltage (LD, $47 \mu \mathrm{m}$ core detector, double GRIN lenses)

4.35 Through-Wafer Power (LD, $47 \mu \mathrm{m}$ core detector, double GRIN lenses)

4.36 Through-Wafer Voltage (LD, $8 \mu \mathrm{m}$ detector fiber, double GRIN lenses)

4.37 Through-Wafer Power (LD, $8 \mu \mathrm{m}$ detector fiber, double GRIN lenses)

4.38 Area of Incorrect Scans

4.39 Static Displacement Output Analysis 
4.46 Output Comparison at $2.2 \mathrm{kHz} \quad 97$

$\begin{array}{ll}4.47 \text { Output Comparison at } 5 \mathrm{kHz} & 97\end{array}$

4.48 Modulation Depth: Frequency Variation 98

4.49 Output Comparison at $10 \mathrm{~V}_{\mathrm{pp}} \quad 99$

$\begin{array}{ll}4.50 \text { Output Comparison at } 15 \mathrm{~V}_{\mathrm{pp}} & 100\end{array}$

$\begin{array}{ll}4.51 \text { Output Comparison at } 30 \mathrm{~V}_{\mathrm{pp}} & 100\end{array}$

4.52 Modulation Depth: Voltage Variation 101

5.1 Loss Areas in an Integrated Through-Wafer Optical Monitoring System 104

B.1 Polysilicon Stress Trends 117

B.2 Polysilicon Resistivity Trends $\quad 118$ 


\section{List of Tables}

3.1 Flexure and Spring Constant Data for Geometry B 37

3.2 Flexure and Displacement Data with V = 30V, Geometry B 38

3.3 Flexure and Frequency Data for Geometry B 39

3.4 Voltage and Displacement Data with Length $=300 \mu \mathrm{m}$, Geometry B 40

3.5 Flexure and Displacement Data with V =20V, Geometry A 42

3.6 Flexure and Frequency Data for Geometry A 43

3.7 Voltage and Displacement Data with Length $=450 \mu \mathrm{m}$, Width $=2 \mu \mathrm{m}$, Geometry A

3.8 Flexure and Displacement Data with V =20V, Geometry C 46

3.9 Flexure and Frequency Data for Geometry C 47

3.10 Voltage and Displacement Data with Length $=450 \mu \mathrm{m}$, Width $=2 \mu \mathrm{m}$, Geometry C

3.11 Device Parameters $\quad 49$

4.1 Transmission Percentages $\quad 84$

5.1 Integrated Optical Monitoring System Transmission Percentages 104

B.1 MUMPS 20 Film Parameters 117 


\section{Chapter 1}

\section{Introduction}

Current trends in sensing, display, and control applications have certain functionality requirements that make the implementation of microoptoelectromechanical systems (MOEMS) necessary. Complete pre-assembled MOEMS on silicon integrated circuits can be created by combining MEMS with integrated optical elements to integrate the coordinated movements of microactuators with optical components such as mirrors and diffraction gratings. Systems such as these carry several advantages including the lack of need for component alignment, mass production, and high packing density. They are small, lightweight, and offer low power operation [1]. The greatest advantage is the ability to directly integrate MOEMS with electronics to produce sophisticated devices. These micron to submicron scale devices utilize lateral, rotational, and torsional movements to perform the desired operations in a system. Using device geometries that take advantage of these types of movements, a myriad of different sensing and actuation tasks can be realized.

Due to the small range of movement exhibited by MEMS devices, the continuous measurement of these movements for control purposes over the MEMS lifetime poses difficulties. Stroboscopic techniques and laser interferometry methods have been used to 
evaluate the movements of MEMS [2], [3]. Due to the limited accessibility of the devices in embedded systems, the aforementioned monitoring methods, used primarily in research settings, often cannot be used. Electrical means of sensing and predicting device movement involving capacitance changes have been explored as well [4], [5], [6]. While easier to integrate into a MEMS package, the signal change associated with device movement is small, has little dynamic range, and may be lost in the noise created by the higher voltages used to power the device. Other problems occur when the measuring device affects the performance of the system itself [7]. In certain cases, monitoring the device's movement causes its behavior to change. A system to acquire a strong movement signal as well as relay this information that could be fabricated at the same time as the devices themselves could eliminate these problems in the same manner that alignment problems are eliminated in combining MEMS and integrated optical elements in MOEM systems.

Through-wafer optical interconnects offer significant benefits in the implementation of multicomputer interconnection networks including added dimensionality, high bandwidth, and compact packaging [8]. Interconnects such as these can be employed to realize the goal of adding an optical monitoring and control system within specific MEMS. A basic system could use planar or through-wafer optical interconnects to optically probe the device, sense the movement, collect the beam, and optically feed back this information to control circuitry. This approach involves multiple optical layers to route the infrared beam to the desired areas. The movement of the device would cause it to interfere with the beam in a way to change its intensity or phase. This 
change in the beam parameters could be sensed and sent to control circuitry area on the same chip where microstructure drive corrections can be made as necessary.

The focus of this research is to characterize and evaluate a through-wafer method of monitoring MEMS device motion using lateral comb resonators as an initial research testbed. This monitoring method is shown to provide a large change in output signal when an optical probe is interrupted by the movement of the translation stage of the device. The output signal will then be used to validate theoretical characteristics of the moving structure. Methods of implementation of a device level system using planar folded and integrated diffractive optics will also be explored.

\subsection{Microelectromechanical Systems}

In order to understand the context in which this research is being done and its range of impact, a brief representative review is given of MEMS technology and the role of position monitoring. The need for optical monitoring and feedback control becomes apparent when MEMS devices are used individually or in large arrays to achieve a desired sensing or actuation task.

\subsubsection{Translational MEMS}

The most common type of MEMS devices are those of the translational variety. One such device, the lateral comb resonator has been employed in many applications where lateral movement is needed. This device often has two sets of stationary comb fingers interleaved with comb fingers on both sides of a translation stage situated between the stationary combs. These interleaved fingers cause an electrostatic force when 
a voltage is applied. To counteract this force a mechanical force is provided by spring structures, or flexures, attached to the translation stage. The combination of these two forces allows the stage to move in one direction and then return to its original position when the voltage is removed. Figure 1.1 shows and example of a translational MEMS device.

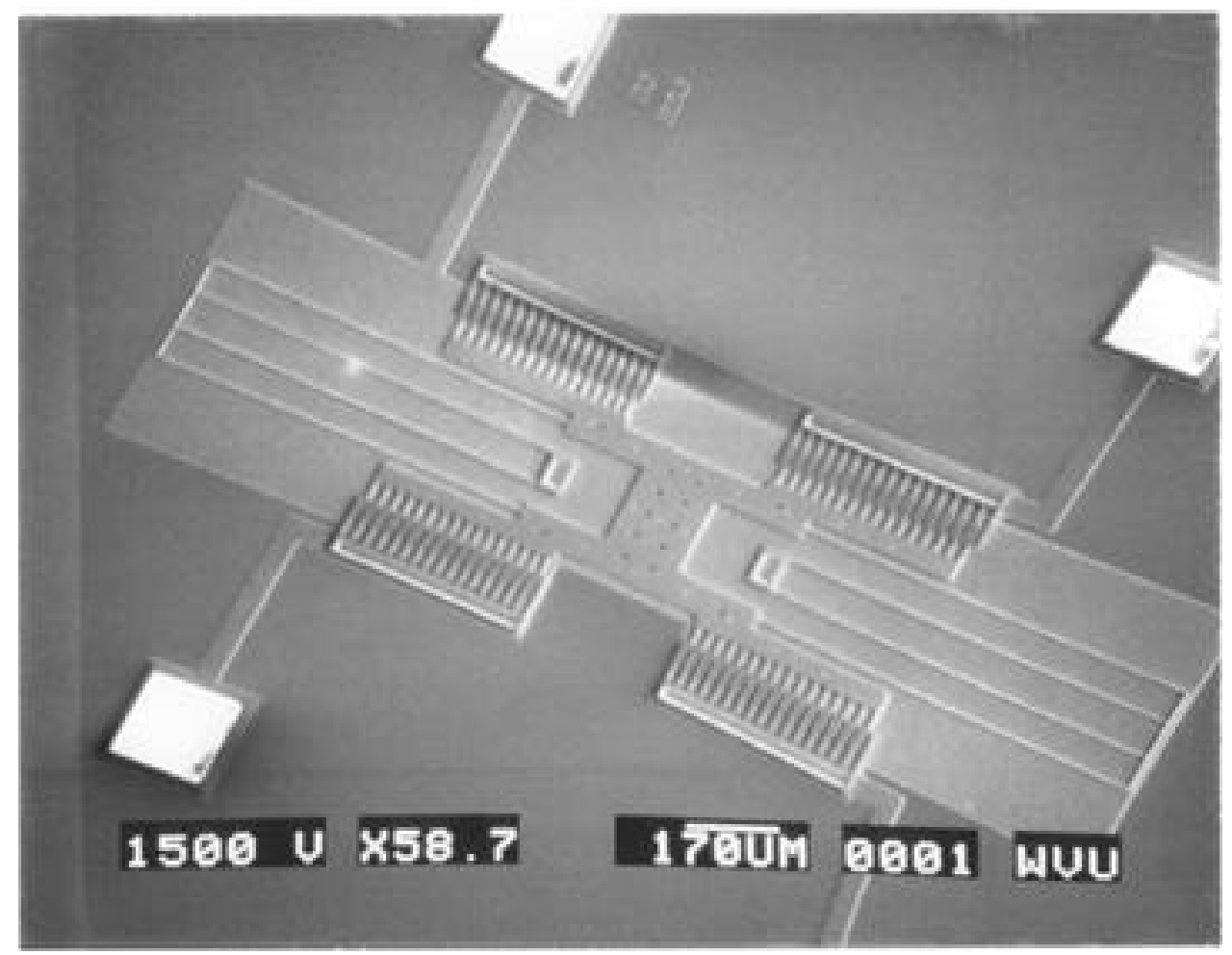

Figure 1.1: Lateral Comb Resonator Designed at WVU

Optical methods of motion monitoring are most suitable in devices such as this that exhibit continuous movement. Due to the simplicity of their movement, resonator devices have been used in many applications involving the actuation of components in a MOEM system. One such system employs translation stages in order to position components in a 
surface-micromachined free-space optical bench [9] (Figure 1.2). Hinge structures can be fabricated that allow elements to be positioned at an angle to the plane of movement. By moving two mirrors situated at $45^{\circ}$, the beam from the semiconductor laser can be scanned in two dimensions. Other structures such as micro-Fresnel lenses can be made if focusing or further direction of the beam is needed.

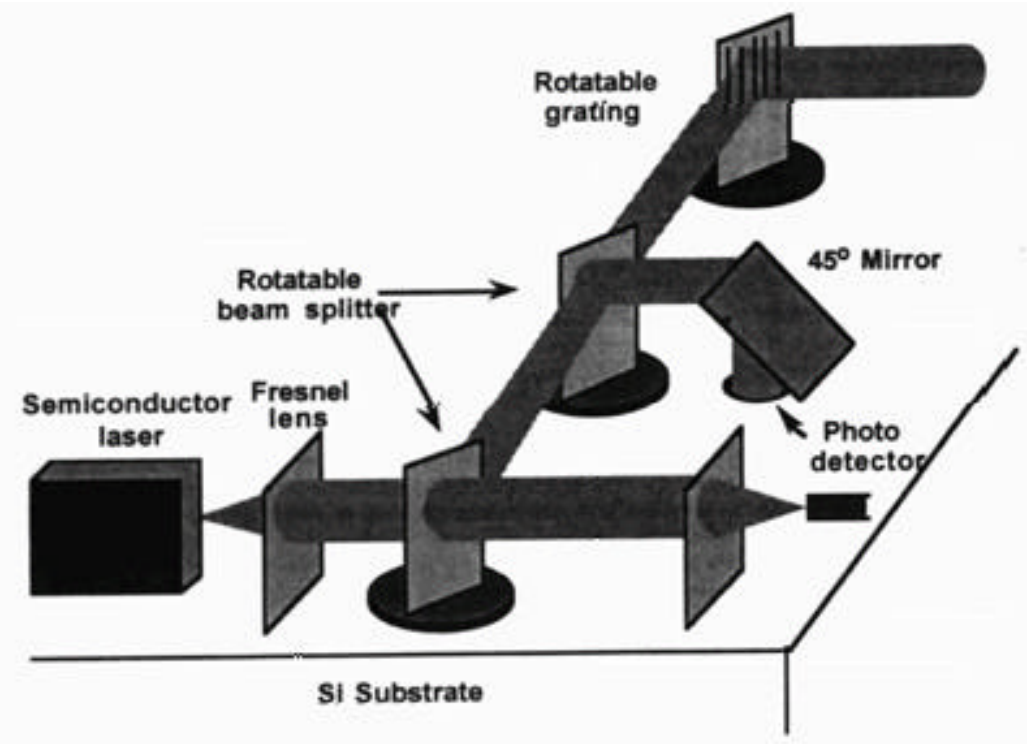

Figure 1.2: Free-Space Microoptical Bench [9]

Another application of linear resonators is in the area of optical switching. A translation stage with an upright mirror fabricated on it has been used to create a monolithic (single chip) $2 \times 2$ optical bypass switch for a fiber data distribution interface (FDDI) local area network (LAN) [9]. This free-space approach to switching can reduce insertion losses and cross-talk associated with conventional switching methods. The two states of the switch, CROSS and BAR, allow for failed nodes in the system to be bypassed (Figure 1.3). Because the comb resonators in this and the previous system 
exhibit only limited ranges of motion, mechanical limit sensors rather than continuous optical monitoring methods may be employed to ensure proper device operation.

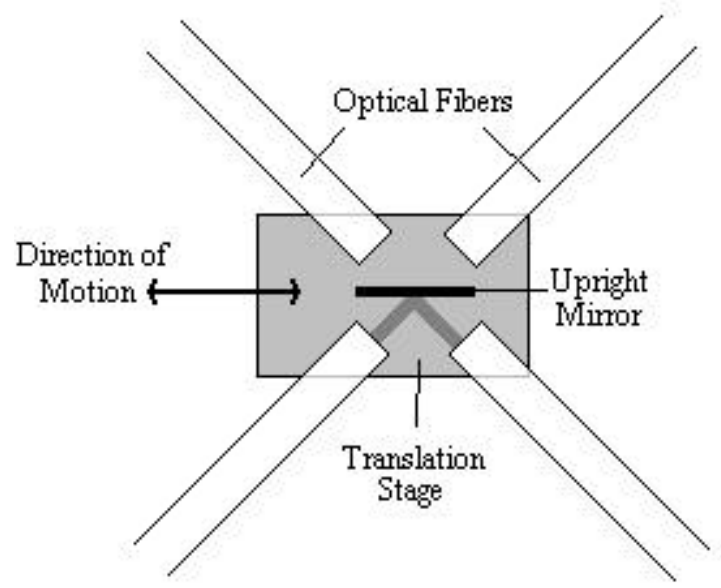

Figure 1.3: Optical Switch [9]

\subsubsection{Torsional/Vertical Motion MEMS}

Micromirror arrays utilize MEMS technology in order to scan or modulate light. Linear arrays of such mirrors are useful for light modulation in printers. These mirrors are rotated torsionally (tilted) by electrostatic attraction using electrodes under each side. The space etched between the mirrored surface and the underlying electrode layer determines the maximum angle of rotation. Each mirror, or pixel, is individually controlled by its location, or address, on a MOS chip. This individual addressability makes the array a highly tunable device.

Many free-space optical systems, such as astronomical telescopes and line-ofsight optical communications, experience irregularities that effect the received signal. These irregularities, or aberrations, are usually caused by free space turbulence, such as thermal distortion. Aberrations cause a fuzziness of the received signal, creating a level of uncertainty that could cause major problems in high precision applications. With the 
growth of integrated optics comes the possibility to reduce or even eliminate such problems. By coating the surface of the image or receiving plane with thousands of integrated deformable mirrors, these aberrations can possibly be reduced. An array of these devices has tested and characterized by various research groups [10]. The goal of the deformable mirror array is to decrease the intensity of the fringes caused by diffraction, and increase the intensity of the $0^{\text {th }}$ order maximum to clarify the signal or image. Another application uses a continuous-membrane mirror with pistons or flexures (springs) that can change the curvature of the mirror when voltage is applied (Figure 1.4) [11]. Again, the resolution is determined by the size and density of the mirrors in the array.

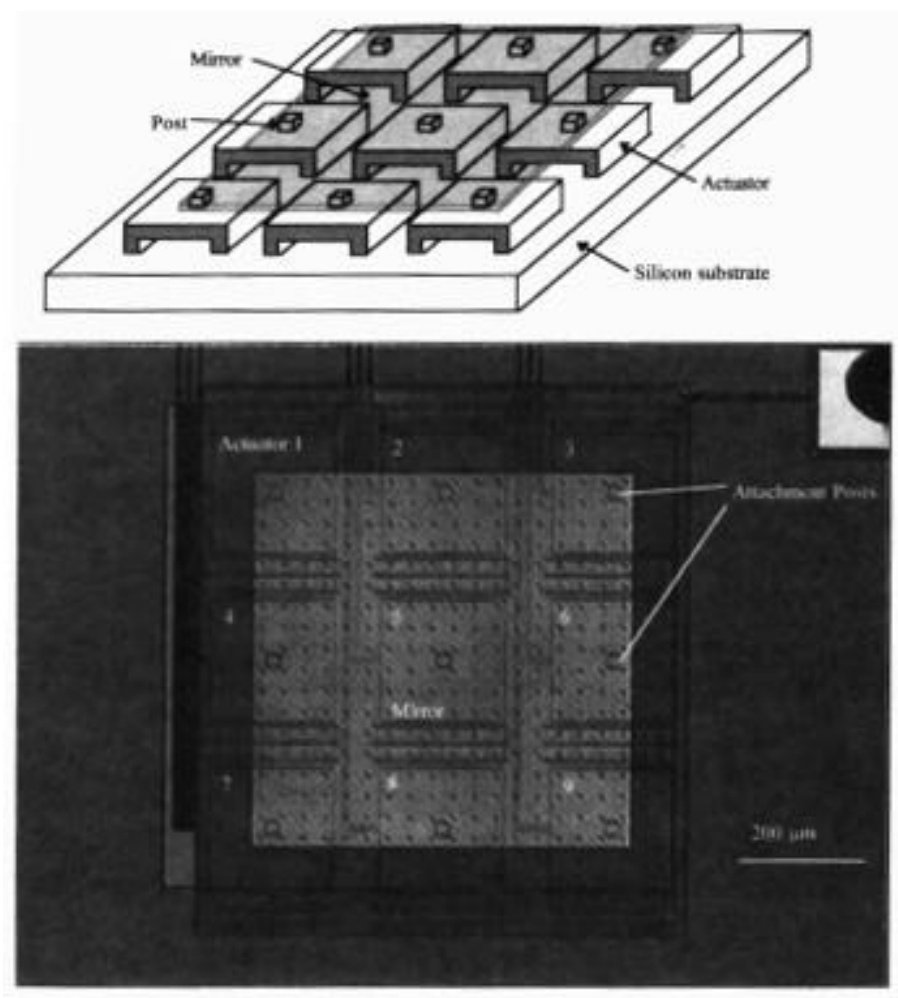

Figure 1.4: Deformable Micromirror [11] 
Digital communication is causing a need for more efficient ways to display the information being sent. The natural interface to digital data is a digital display. This type of display accepts an electrical signal of bits and changes them to optical bits as output. Texas Instruments is the forerunner of this new display technology known as a DMD (digital micromirror device). There are three aspects that make this technology desirable: the combination of audio, text, graphics, and video; high fidelity data transmission, storage, and playback; and limitless reproduction without degradation. The DMD is made up of an array of tilting micromirrors fabricated over a CMOS memory structure (Figure 1.5) [9]. Because of the geometry, the mirror has a maximum translation angle of \pm 10 degrees. When the memory cell is in the (1) state, the mirror tilts to +10 degrees, and tilts to -10 degrees in the (0) state. After combining this structure with a light source and projection optics, (1) corresponds to a bright area and (0) corresponds to a dark one. With binary pulse width modulation, a gray scale is achieved, and color can be added using color filters and multiple DMD chips. 


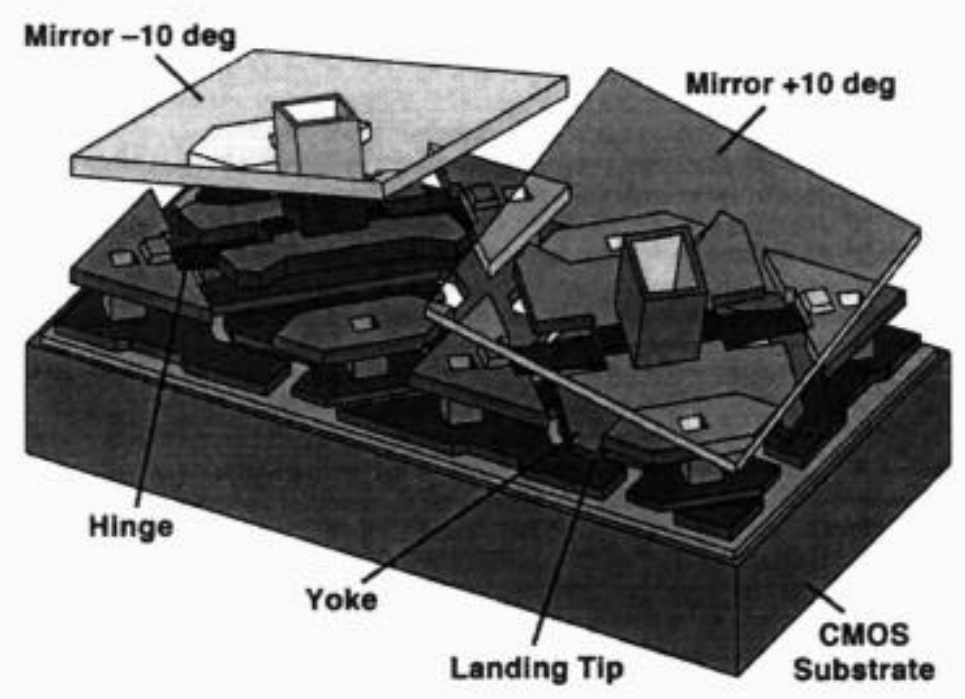

Figure 1.5: Digital Micromirror Display (DMD) [9]

All of these devices use close proximity to increase resolution. This resolution will be diminished if a large number of the devices are behaving improperly or not working at all. Here, the complexity of the device manufacturing process may not lend well to cointegration of the optical monitoring system. Electrical monitoring methods may be more suitable, especially since each pixel is a binary position device, lending well to limit sensing.

\subsubsection{Gear/Motor Rotary Motion MEMS}

The other major type of MEMS structure includes those that rotate in the plane of fabrication. Rotation can be achieved by varying the voltage of the "stator" electrodes situated around rotor electrodes with the opposite voltage applied. This variation causes a repulsion or attraction, much like that exhibited by linear comb resonators, except in a rotational direction. Another method of achieving motion is to combine gears with 
translational MEMS, such as the Sandia Microengine [12]. One lateral resonator device seen in Figure 1.6 operates out of phase with the second to cause a circular action of the center geared device.

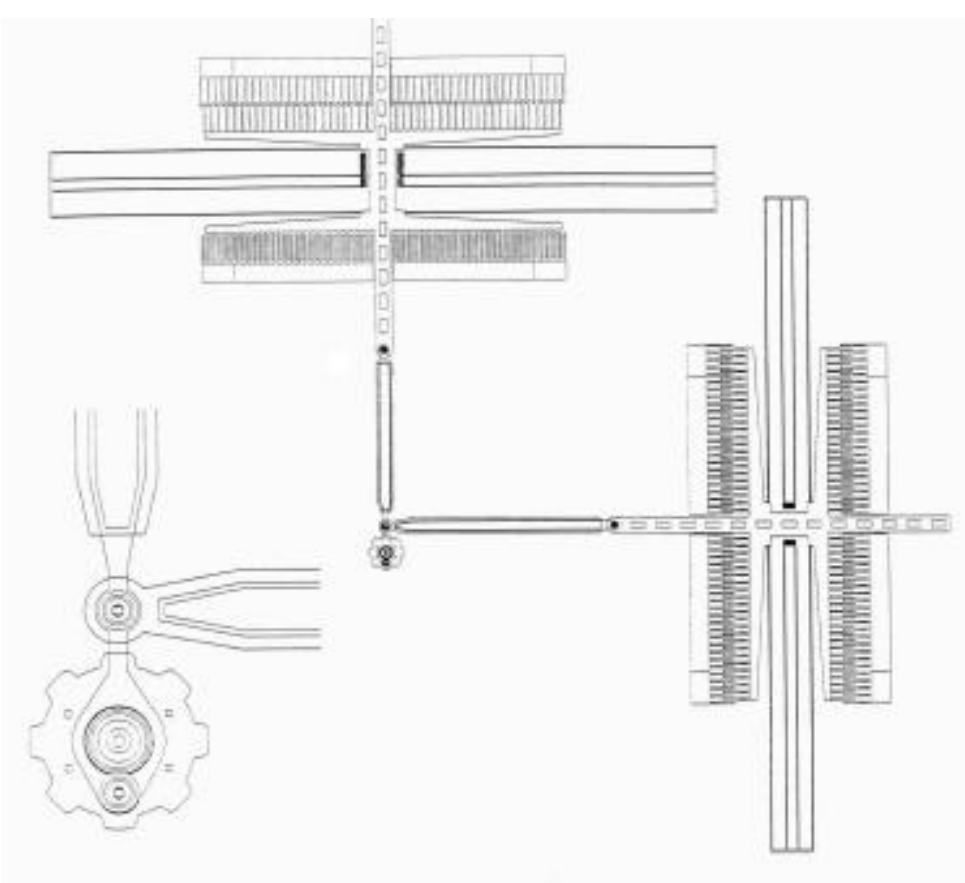

Figure 1.6: The Sandia Microengine

These devices have been developed as arming devices on nuclear warheads. Devices such as these that exhibit continuous movement need a reliable system of microstructure monitoring if they are to be applied in safety critical systems. Optical methods that give a high output signal would surely make these systems more reliable.

Rotational MEMS can be employed to give another degree of freedom to the free space optical bench [9]. Instead of being confined to lateral motion, the micromachined optical components can be rotated $360^{\circ}$ if desired. Like their translational counterparts, this type of MEMS has been used in the areas of optical switches and planar scanning. The rotor of an electrostatic polysilicon micromotor can be fabricated with a diffraction 
grating on it [1]. This grating can consist of two different spatial periods to redirect light at two different angles. Again in all cases, knowing the frequency of rotation of the rotor is important to ensure reliability.

\subsection{Optical Interconnect Technologies}

Optical interconnects have been the focus of many research groups because of the benefits they bring to low level microelectronic systems. They are of interest in this research work as a means to realize optical monitoring of MEMS devices. These types of interconnections show promise for both high dimensionality and inter-chip bandwidth, and more compact packaging [8]. Effective use of light to perform low-level processes also can decrease on-chip capacitance and power consumption. Parallel interconnects, such as slab and strip waveguides, route light on the surface of the chip. Perpendicular, or through-wafer, interconnects route light through the silicon substrate, a desirable trait for the implementation of multi-chip modules (MCM's) [13], [14]. Integrated diffraction and refraction gratings can be combined with parallel and through-wafer interconnects to form planar folded optical systems [15], [16]. The structures used to create both waveguides and through-wafer interconnects can be fabricated after the devices, allowing the bulk fabrication processes of the circuitry on the chip to remain unchanged. The following is an examination of a small sample of current applications of the aforementioned optical interconnect applications. 


\subsubsection{Optical Waveguides}

The two major types of waveguides, slab and strip, use a layer or line of low loss material to route light in one plane. As long as light incident on the interior walls of the waveguide is greater than the critical angle, light will propagate with little loss through total internal reflection (TIR). These light guides can support multiple modes of input wavelengths, or be designed to be limited to a single mode of propagation only. Polymer materials have been developed that provide low loss propagation over distances relatively large compared to the dimensions of the guide itself [17].

Strip waveguides have been implemented for use in integrated interferometers. These devices use a change in the intensity or phase of a light beam to perform a variety of sensing tasks. One group has fabricated strip waveguides in a Mach-Zender configuration to create an integrated pressure sensor [18]. A cavity was etched under one branch of the interferometer. Pressure applied to the top surface causes the index of refraction of the branch with the cavity under it to change, creating a delay in the light passing through it. This output is then coupled to a fiber connected to a circuit calibrated to give a pressure that corresponds to the change in phase of the light.

Another group applied waveguides in a Michelson arrangement to create an integrated displacement sensor [19]. Light that is input to two separate branches, a measurement beam and a reference beam, are sent to outputs. The measurement beam is reflected off of the object whose displacement is being measured. Both beams are routed back to a detector fabricated on the chip. Differences in intensity are compared and calibrated to correspond to a physical change in the displacement of the object. Slab 
waveguides have also been employed to perform similar interferometry processes for evanescent wave sensing [20].

\subsubsection{Through-Wafer Interconnects}

As mentioned before, optical methods of interconnection can provide added dimensionality to microelectronic systems. The majority of on-chip processes occur only in two dimensions. A third vertical direction of connection between different layers of circuitry would allow for larger amounts of parallel processing. Vertical electrical connections are feasible, but require more and complex processing steps and are undesirable due to the difficulty in repairing such a connection. Vertical optical connections are more appealing because little or no mechanical connection is necessary, and any processing steps should be compatible with present VLSI methods [21]. Areas of interconnection could be planned for beforehand and holes could be etched in the substrate to accommodate the connection. The connection between layers of circuitry could be achieved be vertical-cavity surface-emitting lasers (VCSEL's), detectors, and focusing microoptical elements mentioned earlier [22].

To simplify the vertical connection process further, light could be passed through the substrate material so no holes would be needed. Silicon is transmissive to wavelengths of light beyond its absorption cutoff (> $1.3 \mu \mathrm{m})$. Emitters fabricated below the substrate that operate in this range can be coupled to detectors above the chip. This has been achieved using InP-based emitters and detectors [23]. By stacking several layers of silicon circuitry and implementing these emitter-detector pairs between each layer, high-throughput architectures can be achieved [24]. To implement this type of system, 
the backside of the wafer must be polished in order to make it optically smooth and to reduce transmission losses into the silicon substrate. One drawback of through wafer interconnection is the difficulty in alignment between the emitters and detectors.

\subsubsection{Diffractive/Refractive Optics}

Integrated optical elements that focus and direct light have been incorporated in optical interconnect systems as well. These elements used in conjunction with glass TIR substrates form planar folded optical systems that not only route light in one plane, but also couple it between planes [15]. Diffractive elements, such as integrated Fresnel zone plate lenses have been used for free-space optical connections and in through-wafer applications to both focus and collimate beams [25], [26], [27], [28]. Beam focusing onaxis and off-axis has been explored as well [29], [30]. Incorporating these elements in an integrated optical system requires precise alignment and near flawless device fabrication for acceptable performance.

\subsection{Optical MEMS Device Monitoring}

The emergence of MOEMS has resulted in the possibility for integrated optical elements to be combined with MEMS to create micron scale electro-optical systems. It is the next progression for integrated optics to be combined with MEMS not only to perform needed tasks, but also to monitor the system as well. Two methods have been suggested to optically monitor MEMS device motion. One involves using strip waveguides to route light to the planar edge of a moving device to sense its movement. 


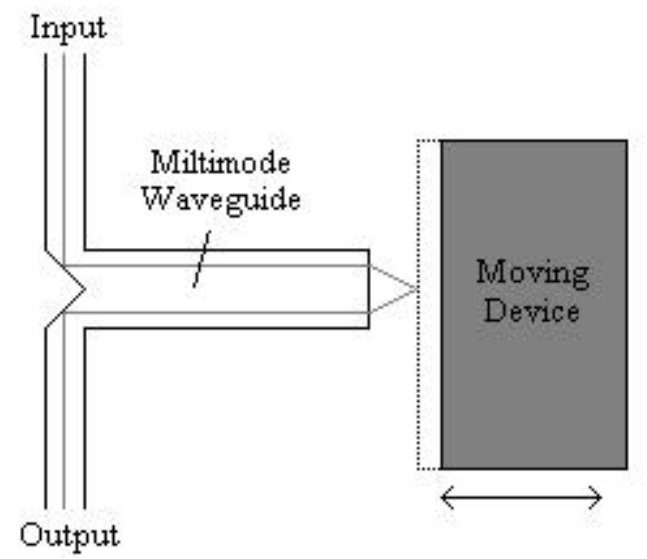

Figure 1.7: Planar Device Monitoring

This method would involve depositing a layer of polymer material over the device layer in which the waveguides would be fabricated. Light would be routed parallel to the devices in the system from the moving stage or rotor to the control circuitry.

Another method of optically monitoring MEMS devices uses a plane waveguide above the device layer and integrated Fresnel lenses.

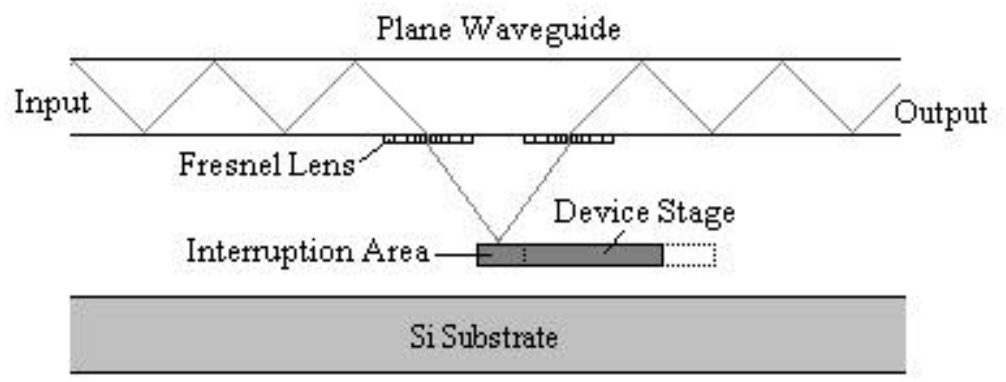

Figure 1.8: Diffractive Optics Device Monitoring

This setup uses the Fresnel lenses to direct light on to the moving device stage. When the stage moves into the beam interruption area, the output signal is coupled back into the 
waveguide by a second lens. This method would be rather difficult due to alignment problems in coupling the reflected probe beam.

Another method, the one investigated in this research, involves using throughwafer free space optical interconnections to monitor the devices.

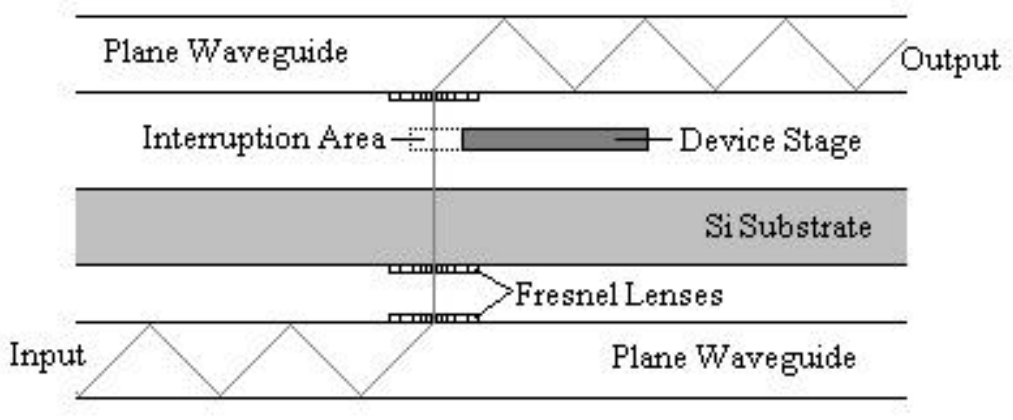

Figure 1.9: Through-Wafer Device Monitoring

Two TIR glass substrates, one above the device layer and one below the substrate, route light to and from the device. Light is coupled between layers by integrated Fresnel lenses that direct the light directly through the silicon substrate. This light is interrupted by the moving device, causing a change in its intensity. The measurement of this change in intensity should be a direct measurement of the frequency of motion of the device and its position if calibrated with its metrology.

This research work reflects an effort to optically monitor and characterize the lateral motion of a lateral comb resonator by using an infrared beam directed through the silicon substrate and focused in the device plane to gain a better understanding of the device's behavior. The output signal will be monitored by a detector located above the device plane. Once the optically monitored signal is determined, control methods may 
later be implemented to correct the movement of the device if it is not performing correctly.

The through-wafer method chosen to monitor the device offers many experimental challenges that must be met before a meaningful signal can be obtained. Infrared alignment, due to the "invisibility" of the light being used, is not straightforward. Transmission losses and the scattering of the beam by the device geometries also pose a problem. Small spot sizes are needed, but achieving a small spot and determining its actual size is very difficult because of the methods used to visually monitor the system. Coupling the probe beam to a detector with full adjustability for both, as well as allowing the device to be powered by probe tips creates the need for a complex and unstable experimental setup. Interpreting an output signal with no idea of the area of the device being monitored makes verification of the signal difficult. If these challenges are overcome, the objective of creating a through-wafer method that gives a meaningful signal representing the actuation of a MEMS device will be realized. Results obtained in this research will confirm that an integrated through-wafer optical monitoring setup is warranted, and that integration will reduce many of the problems experienced when using the experimental setup. 


\section{Chapter 2}

\section{Theory}

The material presented in this chapter contains the theory that is the basis for understanding resonant comb drive MEMS device motion, mutilayer transmission, and the optics involved in reducing the spot size of the infrared sources. Section 2.1 deals with the electrophysics of lateral comb resonator MEMS devices with a folded flexure spring design. This research involved the detection of infrared light passed through a silicon wafer with devices fabricated on the surface. Section 2.2 discusses the transmission of a beam of light through multiple layers of semiconductor materials and the conditions that maximize it. Section 2.3 outlines methods to reduce spot size of the fiber output of the infrared sources used to diameters suitable for this application. A brief discussion of beam intensity is given as well. Section 2.4 addresses the optical properties of off-axis Fresnel gratings. 


\subsection{MEMS Motion}

MEMS utilize a combination of electrostatic and mechanical forces in order to cause movement of the released portions of the devices in the system. The lateral comb resonators used in this research gained their electrostatic force from many interleaved comb fingers energized by a voltage source. There are several types of spring structures that can provide the mechanical force for the device. Serpentine, fixed-fixed, crab-leg, and folded flexures have been employed in MEMS devices [31]. Based on initial research by our group, it is determined that the folded flexure design would be the most suitable for this application because of its linear characteristics.

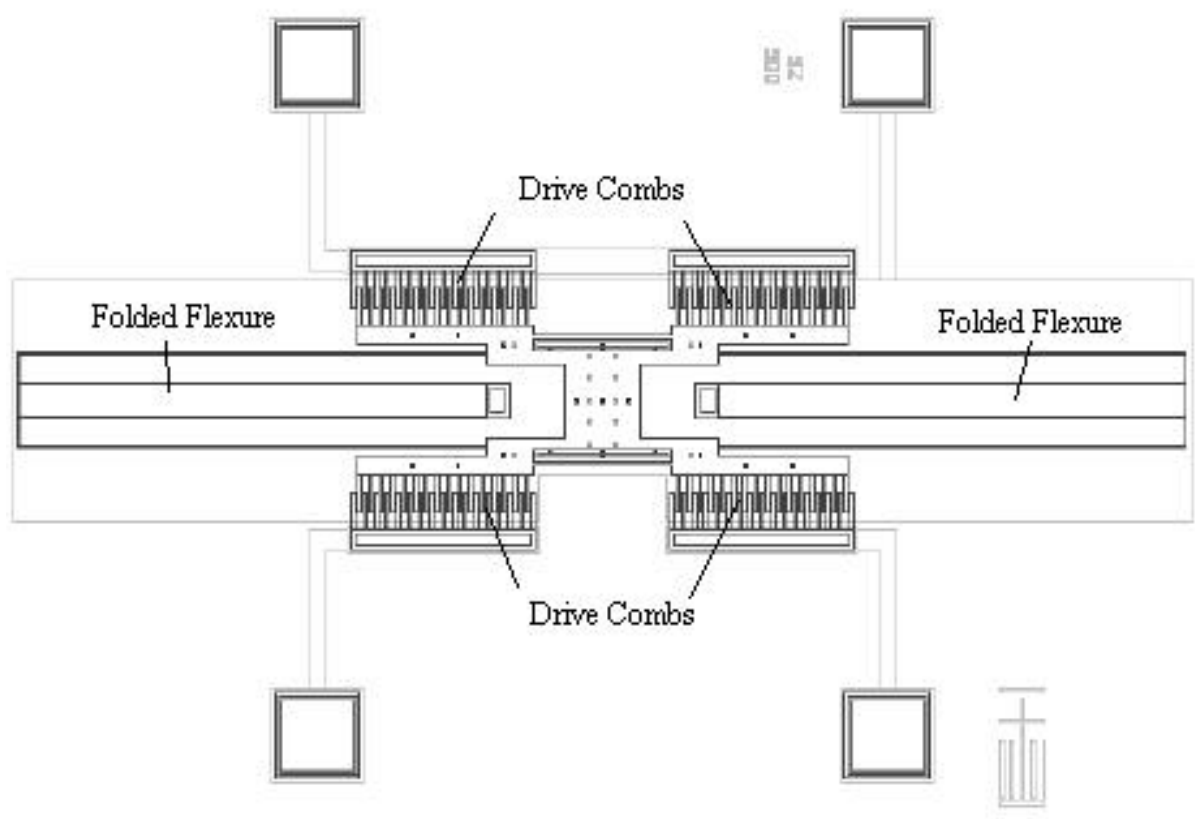

Figure 2.1: Lateral Comb Resonator with Folded Flexure Design 
The spring constant of the folded-beam flexure is [32]

$$
k=\frac{E a^{3} t}{l^{3}}
$$

where $E$ is Young's modulus for polysilicon $(160 \mathrm{Gpa}), a$ is flexure width, $t$ is flexure thickness, and $l$ is flexure length.

For our studies, it was desirable that the center stage of the device should travel as far as possible. Resonant frequency, $\omega$, where maximum lateral displacement occurs, is determined by

$$
\omega=\sqrt{\frac{2 k}{m}},
$$

where $m$ is the mass determined by multiplying the volume of the center stage by the density of polysilicon $\left(2.33 \times 10^{-15} \mathrm{~kg} / \mu \mathrm{m}\right)$.

The two forces mentioned earlier oppose each other to create oscillatory movement of the center stage. The electrostatic force generated by the comb fingers is given by [32]

$$
F_{e}=n \varepsilon \frac{t}{g} V^{2}
$$

where $n$ is the number of fingers creating capacitance, $\varepsilon$ is the permittivity of free space $\left(8.854 \times 10^{-12} \mathrm{C}^{2} / \mathrm{N} \mathrm{m}^{2}\right), t$ is the thickness of the fingers, and $g$ is the gap between stage and drive fingers. 
The force that restores the stage is the spring force of the flexure

$$
F_{s}=k\left(x_{0}-x\right)
$$

where $x_{o}$ is the initial gap distance between stage and stator comb fingers, and $x$ is the distance after deflection.

These forces oppose each other, and when in equilibrium,

$$
2 k\left(x_{0}-x\right)=n \varepsilon \frac{t}{g} V^{2}
$$

This equation can then be simplified to can be simplified to [32]

$$
\Delta x=\frac{n \varepsilon t V^{2}}{2 g k},
$$

giving the expression for displacement of the translation stage.

An analysis of the effects of changing various parameters of the device, such as the mass of the translation stage, the number of comb fingers, input voltage, and flexure lengths, is presented in Chapter 3.

\subsection{Multi-layer Transmission}

Because a through-wafer method of MEMS monitoring involves directing light through a silicon substrate and the devices fabricated on its surface, this section presents the methods of determining the transmittance of a beam directed in such a manner. 
The reflectivity of a simple plane interface at normal incidence is given by [33]

$$
r_{12}=\frac{n_{1}-n_{2}}{n_{1}+n_{2}}
$$

where $n_{1}$ and $n_{2}$ are the indices of refraction of the incident and transmitted media respectively as shown in Figure 2.2.

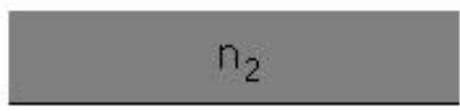

$n_{1}$

Figure 2.2: Plane Interface

The transmittance, $T$, of this interface can be determined by

$$
\begin{aligned}
& R=|r|^{2} \\
& T=1-R .
\end{aligned}
$$

Systems involving multiple layers such as the one shown in Figure 2.2 require an evaluation including the properties of each layer. When examining such systems, not only must the various indices of refraction be considered, but also the thickness of each layer as well. The Jones matrix of a thin homogeneous dielectric film for a TE wave at normal incidence is given by [34]

$$
M=\left[\begin{array}{cc}
\cos \beta & -\frac{1}{n_{f i l m}} \sin \beta \\
-i n_{f i l m} \sin \beta & \cos \beta
\end{array}\right]
$$


where

$$
\beta=\frac{2 \pi n_{\text {film }} h}{\lambda_{0}}
$$

with $\lambda_{0}$ being the wavelength of the beam, $h$ as the thickness of the layer, and $n_{\text {film }}$ its refractive index. This matrix represents the effects that thickness and refractive index will have on the light transmitted at a given wavelength

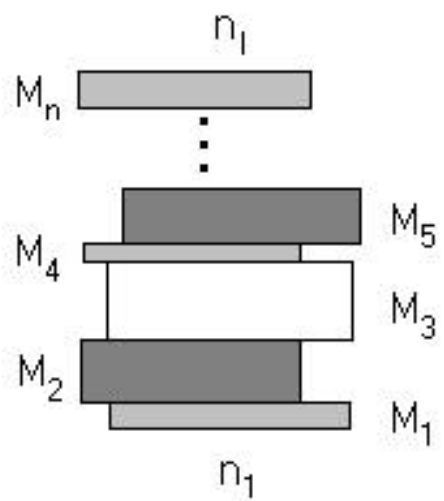

Figure 2.3: Multiple Layers

The characteristic matrix of this entire system is given by [34]

$$
M=M_{n} \ldots M_{5} M_{4} M_{3} M_{2} M_{1} .
$$

The reflectivity for this system, with $n_{1}$ being the index of refraction of the incident medium and $n_{l}$ the index of the final medium, can be expressed generally in terms of the elements of $M$ as [34]

$$
r=\frac{\left(m_{11}+m_{12} n_{l}\right) n_{1}-\left(m_{21}+m_{22} n_{l}\right)}{\left(m_{11}+m_{12} n_{l}\right) n_{1}+\left(m_{21}+m_{22} n_{l}\right)} .
$$


This equation can be rewritten in terms of the Fresnel coefficients for one layer (representing a film between two media) as

$$
R=\frac{r_{12}^{2}+r_{23}^{2}+2 r_{12} r_{23} \cos 2 \beta}{1+r_{12}^{2} r_{23}^{2}+2 r_{12} r_{23} \cos 2 \beta}
$$

where $r_{12}$ is the reflectivity of the first interface and $r_{23}$ is the reflectivity of the second interface, both given by (2.1).

These equations are used in Chapter 4 to quantify the decrease in transmitted beam intensity when the translation stage is present, and to suggest how to improve the transmission of the existing device layers.

\subsection{Optical Probe Beam Generation and Manipulation}

Optical methods used to probe the MEMS device will require significant control over beam location and spot size. In order to achieve a small spot size on the surface of the die device layer in this experiment, a graded-index (GRIN) lens was employed so desirable spot sizes could be obtained. This type of lens was chosen because of limited space in the experimental setup. The following is a review of basic Gaussian beam optics

used. Unlike the laser diode used, the LED output was not exactly Gaussian in nature or monochromatic, but the models presented provide a reasonable estimate of system behaviors. This analysis was performed to determine the distance the source should be separated from the lens in order to get a small image spot, and to approximate the resulting spot size. It also provided some insight into the expected normalized beam intensity at the front of the GRIN lens. 
Figure 2.4 and the following equations illustrate the propagation of a Gaussian beam through a thin lens with inner radius $R$ and outer radius $R^{\prime}$ [35]. The IR output is located at $z=0$, and has a waist size of $W_{0}$. The lens is located a distance of $z$ away from the source.

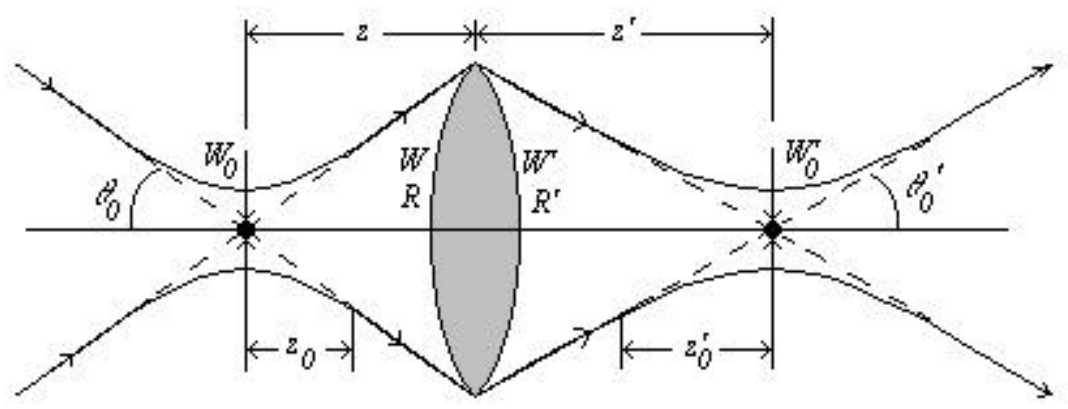

Figure 2.4 Transmission of a Gaussian Beam Through a Thin Lens [35]

The equation that gives the focal length of the lens, $f$, is given by

$$
\frac{1}{f}=\frac{1}{R^{\prime}}-\frac{1}{R} .
$$

The waist radius of the image is given by

$$
W_{0}^{\prime}=\frac{W}{\left[1+\left(\pi W^{2} / \lambda R^{\prime}\right)\right]^{1 / 2}}
$$

where $W$ is the width of the beam at the lens and $\lambda$ is the wavelength of light. The distance of $W_{0}$ away from the lens is determined by

$$
-z^{\prime}=\frac{R^{\prime}}{1+\left(\lambda R^{\prime} / \pi W^{2}\right)^{2}} .
$$


This value is expressed as a negative value because the image lies to the right of the lens. The magnification can be obtained by using [35]

$$
M=\frac{M_{r}}{\left(1+r^{2}\right)^{1 / 2}},
$$

where

$$
r=\frac{z_{0}}{z-f}
$$

and

$$
M_{r}=\left|\frac{f}{z-f}\right| \text {. }
$$

By substituting

$$
R=z\left[1+\left(z_{0} / z\right)^{2}\right]
$$

and

$$
W=W_{0}\left[1+\left(z / z_{0}\right)^{2}\right]^{1 / 2}
$$

into equations (2.3-1) and (2.3-2) respectively, the following beam parameters can be determined [35]:

the waist radius

$$
W_{0}{ }^{\prime}=M W_{0},
$$

the waist location

$$
\left(z^{\prime}-f\right)=M^{2}(z-f),
$$

depth of focus

$$
2 z_{0}{ }^{\prime}=M^{2}\left(2 z_{0}\right),
$$

and the beam divergence

$$
2 \theta_{0}{ }^{\prime}=\frac{2 \theta_{0}}{M} .
$$


The GRIN lens focal length is determined differently from that of a simple thin lens. Figure 2.5 illustrates the use of a single 0.29 pitch GRIN lens to focus the IR output.

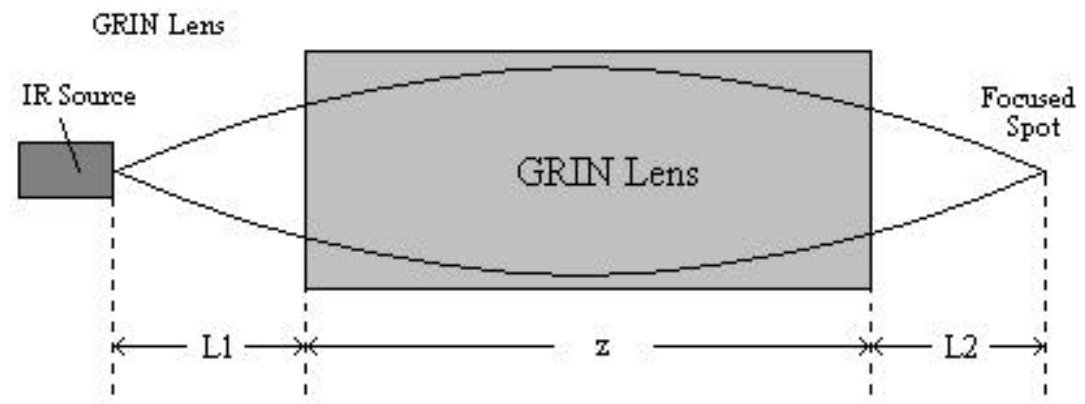

Figure 2.5: GRIN Lens Focal Points [36]

The equation that gives the value of $L 2$ is [36] is

$$
L 2=\left(\frac{1}{n_{0} \sqrt{A}}\right) \cdot\left(\frac{n_{0} \sqrt{A} \cdot L 1 \cos (\sqrt{A} Z)+\sin (\sqrt{A} Z)}{n_{0} \sqrt{A} \cdot L 1 \sin (\sqrt{A} Z)-\cos (\sqrt{A} Z)}\right) .
$$

To focus gain more control over the spot size, two quarter-pitch GRIN lenses can be used. The first lens collimates the IR beam. The second lens sees this beam as a source an infinite distance away, so the spot at the focal distance is approximately the same size and distance away from the lens as the output of the IR source. This configuration is shown in Figure 2.6.

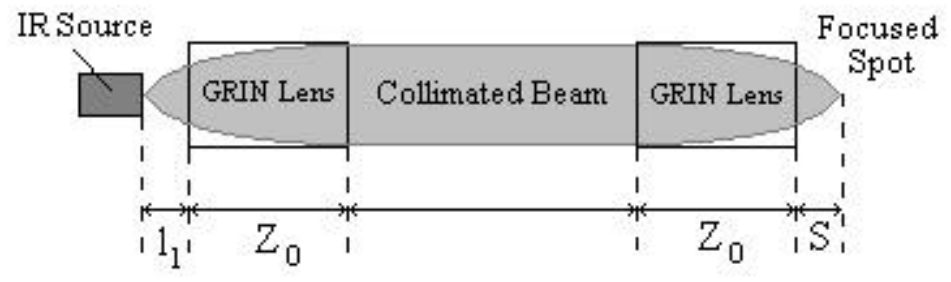

Figure 2.6 Double GRIN Lens Setup [36] 
The focal points for the spot and the working distance of the source are both given by

$$
l_{1}=S=\frac{1}{\sqrt{A} n_{0} \tan \left(\sqrt{A Z_{0}}\right)}
$$

The values of $n_{0}$ and $\mathrm{A}^{1 / 2}$ are both a function of the beam wavelength. For a wide angle SELFOC microlens $(\mathrm{NA}=0.46)$ with a diameter of $2.0 \mathrm{~mm}$ these values are given by [36]

$$
\sqrt{A}(\lambda)=0.2931+\frac{2.369 \times 10^{-3}}{\lambda^{2}}+\frac{7.681 \times 10^{-4}}{\lambda^{4}}
$$

and

$$
n_{0}(\lambda)=1.5868+\frac{8.14 \times 10^{-3}}{\lambda^{2}}
$$

respectively where $\lambda$ is given in microns.

By substituting $L 1$ and $L 2$ into (2.3-11) for $z_{0}$ and $z_{0}$ ' the magnification of the GRIN lens can be determined by

$$
M=\frac{L 2}{L 1}
$$

This value can then be used to calculate the parameters of the image spot.

The infrared sources used in this research had fiber outputs. As the IR output of the LED or laser diode is moved away from the GRIN lens, the beam diameter increases (Figure 2.7). 


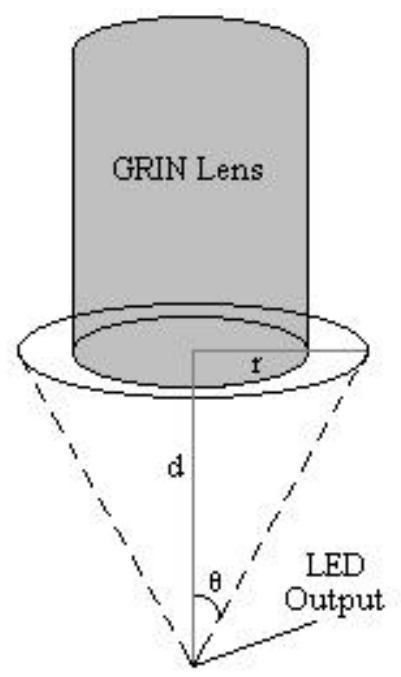

Figure 2.7 Beam Radius at Grin Lens

The spot radius at a certain diameter $d$ can be calculated by

$$
r=d \tan \theta
$$

where $\theta$ is the numerical aperture given by [33]

$$
N A=\left(n_{f}^{2}-n_{c}^{2}\right)^{1 / 2}
$$

where $n_{f}$ is the index of refraction of the fiber and $n_{c}$ is that of the fiber cladding material.

As the spot size increases, intensity, a function of beam radius and axial distance, decreases. This intensity is approximated here as a Gaussian as [35]

$$
I(\rho, z)=I_{0}\left[\frac{W_{0}}{W(z)}\right]^{2} \exp \left[-\frac{2 \rho^{2}}{W^{2}(z)}\right] .
$$


By setting $\rho=0$, the spot intensity at the center can be determined by simplifying equation (2.3-19) to give

$$
I(0, z)=\frac{I_{0}}{1+\left(z / z_{0}\right)^{2}} .
$$

\subsection{Diffractive Optics/TIR Propagation}

In order to implement a through-wafer monitoring system, diffractive optics must be employed to couple light between layers of planar waveguides and focus the optical probe in the device layer of the die. Integrated Fresnel lenses are suitable for this task. The following is a brief consideration of the design and characteristics of both on and off axis Fresnel lenses, and light propagation in a TIR glass substrate.

\subsubsection{On-Axis Fresnel Lenses}

A Fresnel zone plate lens is a set of concentric apertures, each having a thickness that introduces a $2 \pi$ phase shift in light that passes through it. These zones have rotational symmetry. When designing a Fresnel lens for a specific application, certain lens characteristics are dependent on the needed focal length, the size of the lens, and the wavelength of light. The radius, $r_{m}$, of any specific zone is given by [29]

$$
r_{m}=\left[2 m \lambda f+(m \lambda)^{2}\right]^{1 / 2},
$$

where $m$ is the zone number, $\lambda$ is the wavelength of the incident light, and $f$ is the desired 
focal length of the lens. The smallest width of the rings of a Fresnel zone lens is [29]

$$
w=\frac{2 \lambda f}{d L}
$$

where $\mathrm{d}$ is the desired diameter of the lens and $\mathrm{L}$ is the number of phase levels. Increasing the number of phase levels increases the efficiency of the lens, given by

$$
\eta=\left(\frac{\sin (\pi / L)}{\pi / L}\right)^{2}
$$

Figure 2.8 shows both binary (one level) and multi-level Fresnel lens cross-sections.

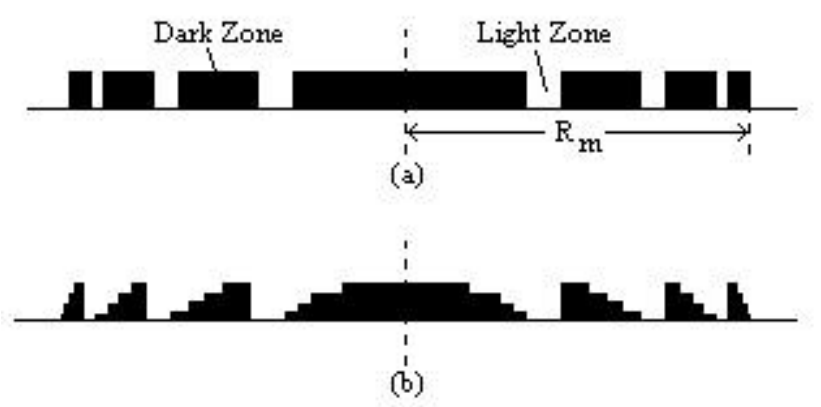

Figure 2.8: Fresnel Lens Cross-Section - (a) binary lens, (b) multi-level lens

\subsubsection{Off-Axis Fresnel Lenses}

Fresnel zone plate lenses that focus or collimate light at an angle to the optic axis can also be designed. Such a configuration is shown in Figure 2.9. 


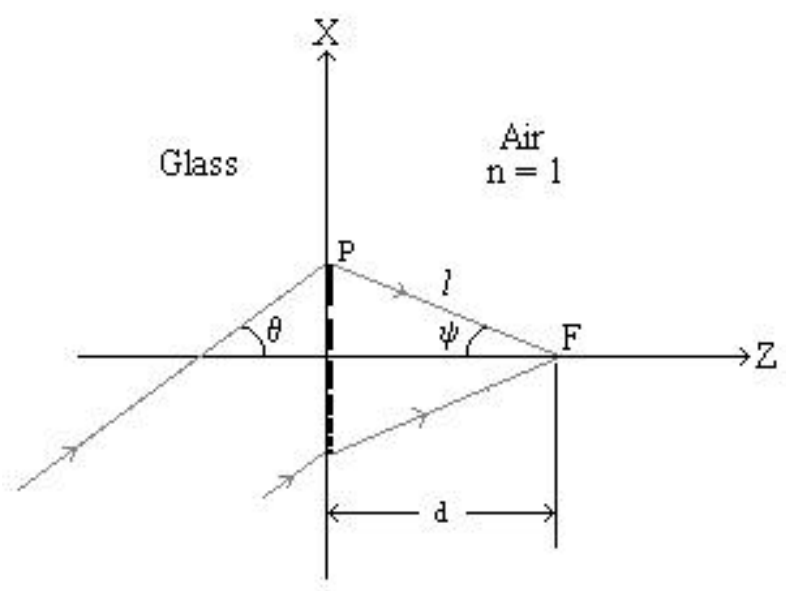

Figure 2.9: Off-Axis Lens Configuration [37]

One implementation of lenses such as these use only a section of the circular ring pattern of regular Fresnel lenses. At point $P$ in the $x-y$ plane of the lens, grating phase is given by [37]

$$
\Omega=\frac{2 \pi}{\lambda}(l+x \sin \theta) .
$$

The wavelength is $\lambda$ and $l$ is the optical path length from $P$ to $F$, which is represented by [37]

$$
l=\frac{n^{2} d}{\sqrt{n^{2}-\sin ^{2} \psi}}
$$

where $d$ is the focal distance and $n$ is the index of the transmitted medium, in this case air. The value of $\psi$ is related to the $\mathrm{x}$ and y coordinates of point $P$ as

$$
\sqrt{x^{2}+y^{2}}=\sin \psi \frac{d}{\sqrt{n^{2}-\sin ^{2} \psi}} .
$$




\subsubsection{Total Internal Reflective Glass Slab Propagation}

TIR glass slabs, like waveguides, can be fabricated out of polymer materials for low-loss light propagation in the medium [17]. A cross-sectional view of a slab waveguide is shown in Figure 2.10.

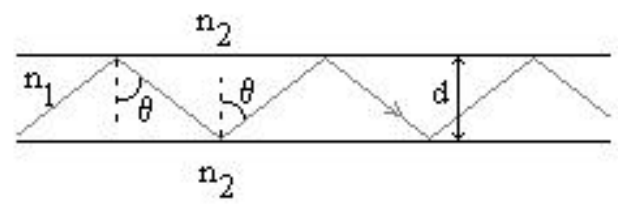

Figure 2.10: TIR Slab Propagation

The critical angle of propagation, $\theta_{C}$, the condition for no leakage of intensity into the surrounding medium, is given by [38]

$$
\sin \theta_{c}=\frac{n_{2}}{n_{1}} .
$$

Any light propagating an angle less than the critical angle will experience a decrease in intensity as it propagates through the medium. 


\section{Chapter 3}

\section{MEMS Design}

Initial stages of this research work involved designing a general-purpose set of MEMS devices for guided wave free-space optical metrology studies. On hand were devices that had been designed by previous graduate students. The original designs utilized a split-comb geometry in order to combine the MEMS structure with integrated optical waveguides for testing. This split in the comb drive allowed a region for the waveguide to be coupled to the edge of the translation stage in order to employ either intensity modulation or interferometry in an attempt to measure the frequency of the movement.

The design employed a folded-beam spring flexure in order to provide the mechanical return force that would counteract the electrostatic attraction of the charged comb fingers. They were analyzed theoretically at lengths up to $150 \mu \mathrm{m}$ and widths of 3 $\& 4 \mu \mathrm{m}$. Each actuator had 32 fingers that were $4 \mu \mathrm{m}$ wide. When fabricated and tested, these structures did not exhibit enough lateral, or translational, movement to be detected when viewed under a microscope. It would be ideal to design the actuator so that lateral movement would be visible when observed under a camera microscope, ensuring that enough movement would be exhibited to cause a definitive change in the intensity of the light used in the waveguide setup. The original design was referred to as Phase I so the 
new designs were labeled Phase II as the next progression toward devices that performed desirably (Figures $3.1 \& 3.2$ ).

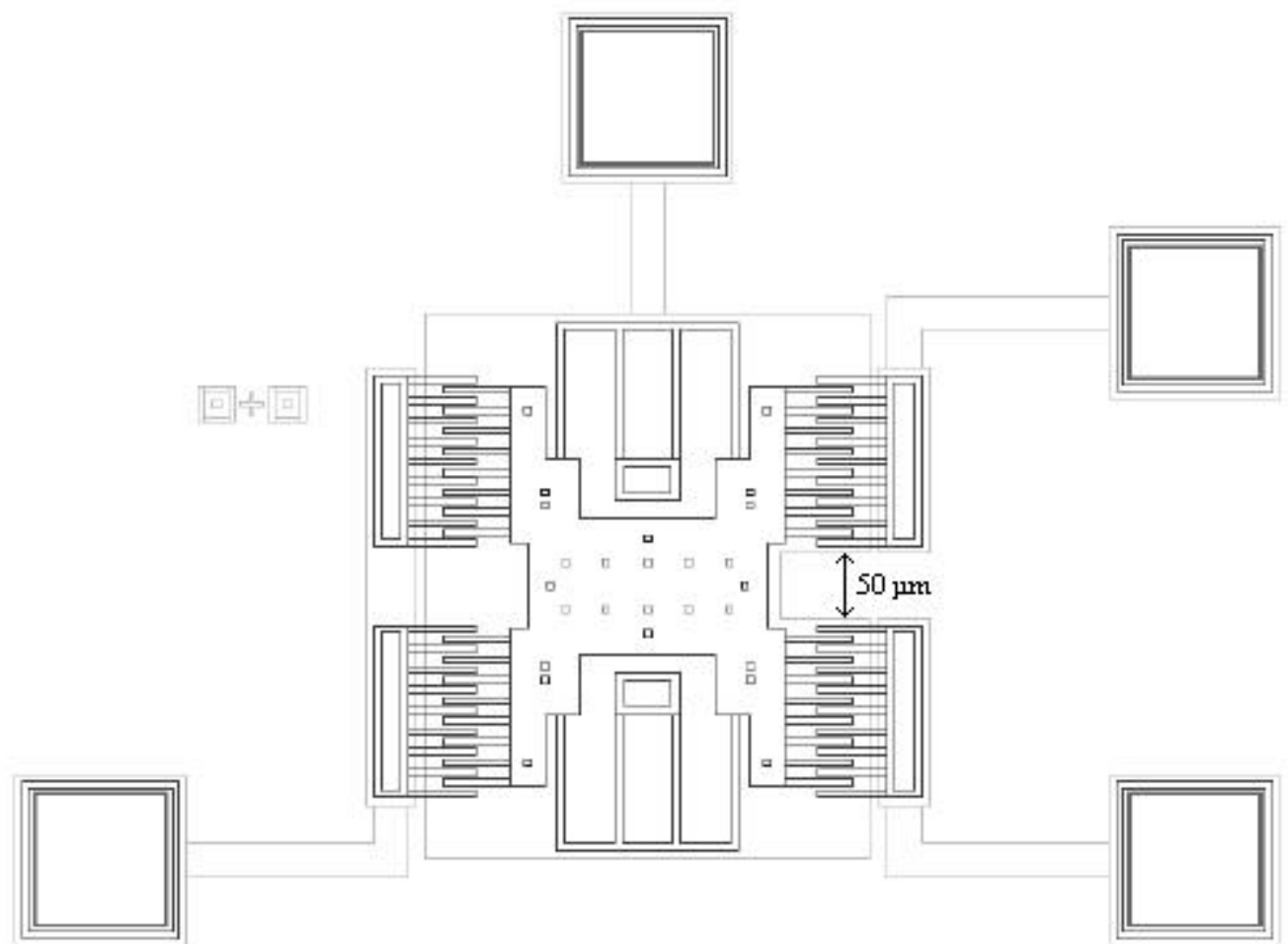

Figure 3.1: Phase I Stage Geometry

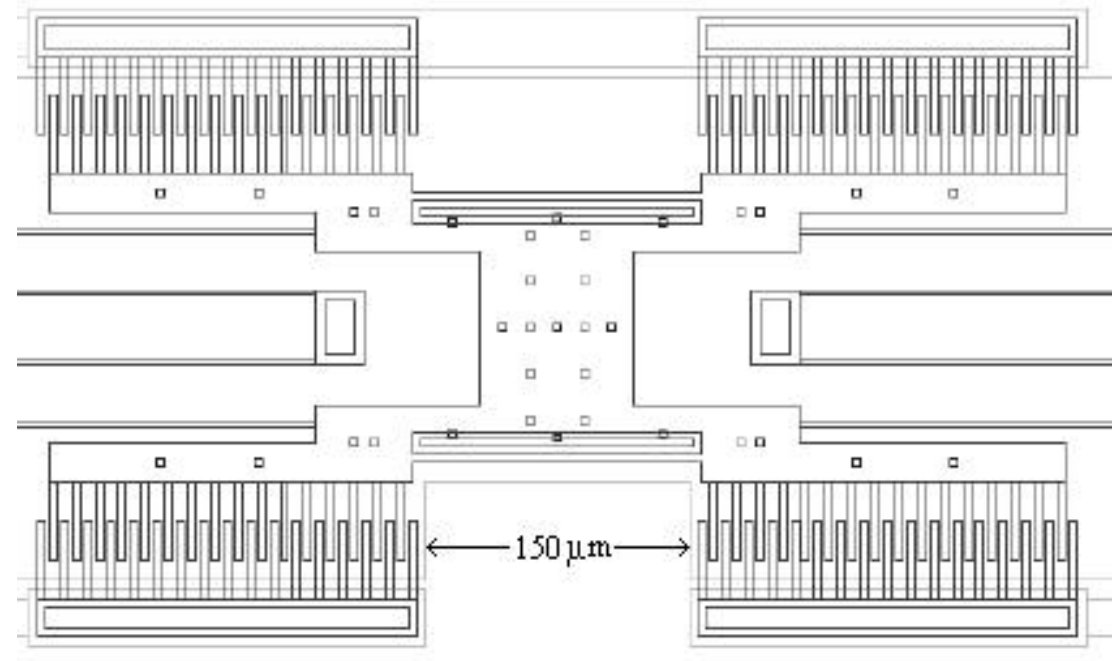

Figure 3.2: Phase II Stage Geometry 


\subsection{Design \& Analysis}

Phase II was initiated in order to design devices performed more desirably. In this phase, the same basic split-comb center stage geometry was kept, and flexure length and width were changed, as well as the number of comb fingers. In order to analyze the dependence of length and width of the flexures on the flexure spring constant, lateral displacement, and resonant frequency, a MATLAB program was written using the equations developed in section 2.1 to analyze the different combinations of parameters. The parameters were determined from the devices as drawn in the CAD tool described in Section 3.2. Actual device parameters will vary due to angled sidewalls and non-ideal thicknesses.

In the program, located in Appendix A, flexure length was varied from 150 to 450 $\mu \mathrm{m}$ and width was $2 \& 3 \mu \mathrm{m}$. Figure 3.3 shows that the $2 \mu \mathrm{m}$ wide flexure has a lower spring constant at all lengths than the $3 \mu \mathrm{m}$ width. It can be observed from Figure 3.4 that the 2 um flexure width should provide greater translational movement at all lengths, and that the longer flexure also increases this movement (up to $~ 9 \mu \mathrm{m} @ 450 \mu \mathrm{m}$ ). This coincides with the spring constant results. Figure 3.5 relates the different resonant frequencies with flexure length at a center stage mass of $1.206 \times 10^{-10} \mathrm{~kg}$ (Geometry B). Figure 3.6 illustrates how increasing the voltage increases lateral displacement at a nominal flexure length of $350 \mu \mathrm{m}$. 


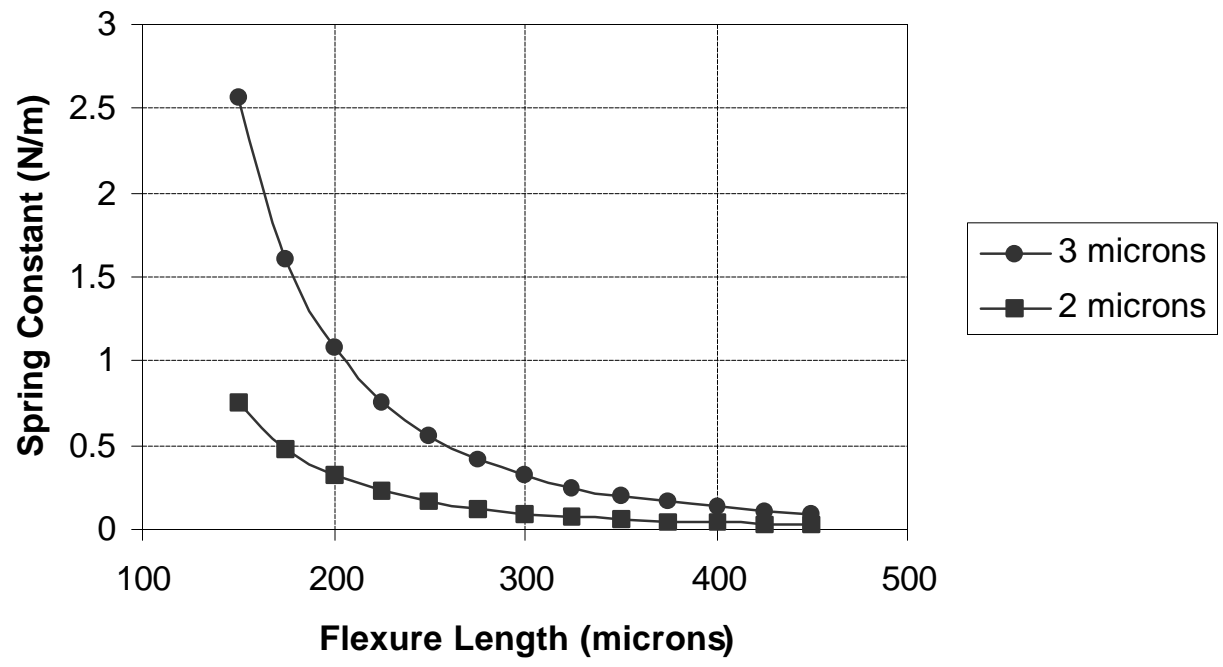

Figure 3.3: Length of Flexure vs. Spring Constant - Geometry B

Table 3.1: Flexure and Spring Constant Data for Geometry B

\begin{tabular}{|c|c|c|}
\hline $\begin{array}{c}\text { Length of } \\
\text { Flexure }(\mu \mathrm{m})\end{array}$ & $\begin{array}{c}\text { Spring } \\
\text { Constant } \\
3 \mu \mathrm{m} \text { width }\end{array}$ & $\begin{array}{c}\text { Spring } \\
\text { Constant } \\
2 \mu \mathrm{m} \text { width }\end{array}$ \\
\hline \hline 150 & 2.560 & 0.7585 \\
\hline 175 & 1.612 & 0.4777 \\
\hline 200 & 1.080 & 0.3200 \\
\hline 225 & 0.7585 & 0.2247 \\
\hline 250 & 0.5530 & 0.1638 \\
\hline 275 & 0.4154 & 0.1231 \\
\hline 300 & 0.3200 & 0.0948 \\
\hline 325 & 0.2517 & 0.0746 \\
\hline 350 & 0.2015 & 0.0597 \\
\hline 375 & 0.1638 & 0.0485 \\
\hline 400 & 0.1350 & 0.0400 \\
\hline 425 & 0.1126 & 0.0333 \\
\hline 450 & 0.0948 & 0.0284 \\
\hline
\end{tabular}




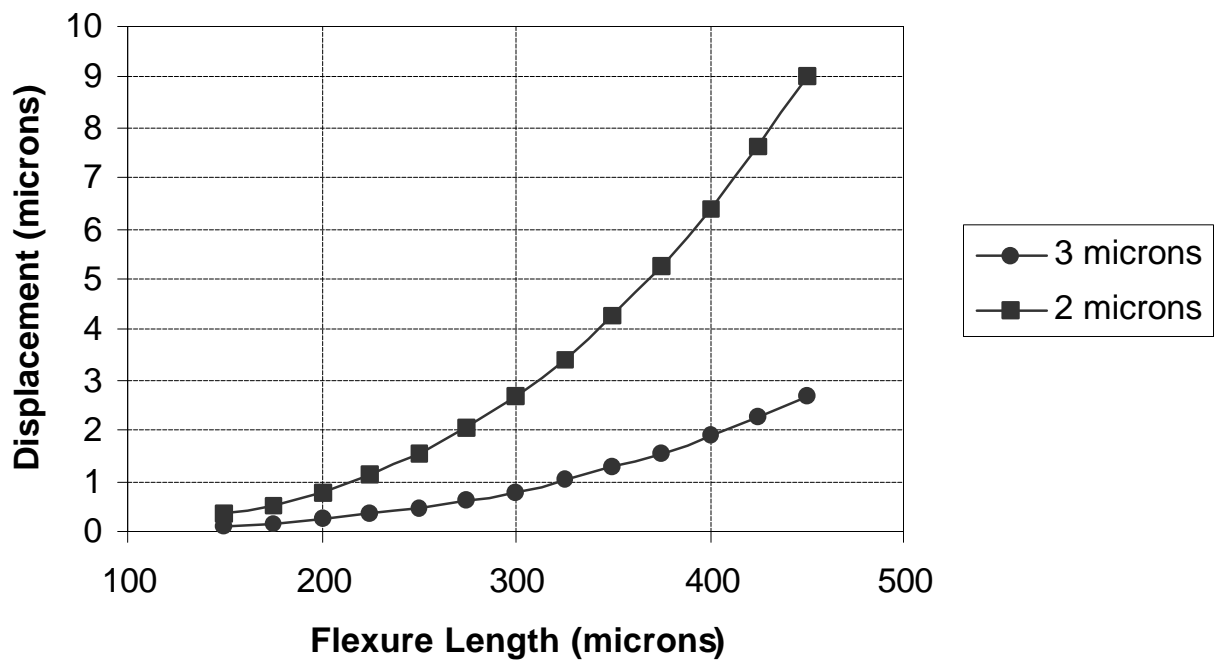

Figure 3.4: Length of Flexure vs. Displacement - Geometry B

Table 3.2: Flexure and Displacement Data with $\mathrm{V}=30 \mathrm{~V}$, Geometry B

\begin{tabular}{|c|c|c|}
\hline \hline $\begin{array}{c}\text { Length of } \\
\text { Flexure }(\mu \mathrm{m})\end{array}$ & $\begin{array}{c}\text { Displacement } \\
3 \mu \mathrm{m} \text { width }\end{array}$ & $\begin{array}{c}\text { Displacement } \\
2 \mu \mathrm{m} \text { width }\end{array}$ \\
\hline \hline 150 & 0.100 & 0.336 \\
\hline 175 & 0.158 & 0.534 \\
\hline 200 & 0.236 & 0.797 \\
\hline 225 & 0.336 & 1.1335 \\
\hline 250 & 0.461 & 1.556 \\
\hline 275 & 0.614 & 2.072 \\
\hline 300 & 0.797 & 2.689 \\
\hline 325 & 1.013 & 3.419 \\
\hline 350 & 1.265 & 4.271 \\
\hline 375 & 1.556 & 5.253 \\
\hline 400 & 1.889 & 6.375 \\
\hline 425 & 2.266 & 7.646 \\
\hline 450 & 2.689 & 9.007 \\
\hline
\end{tabular}




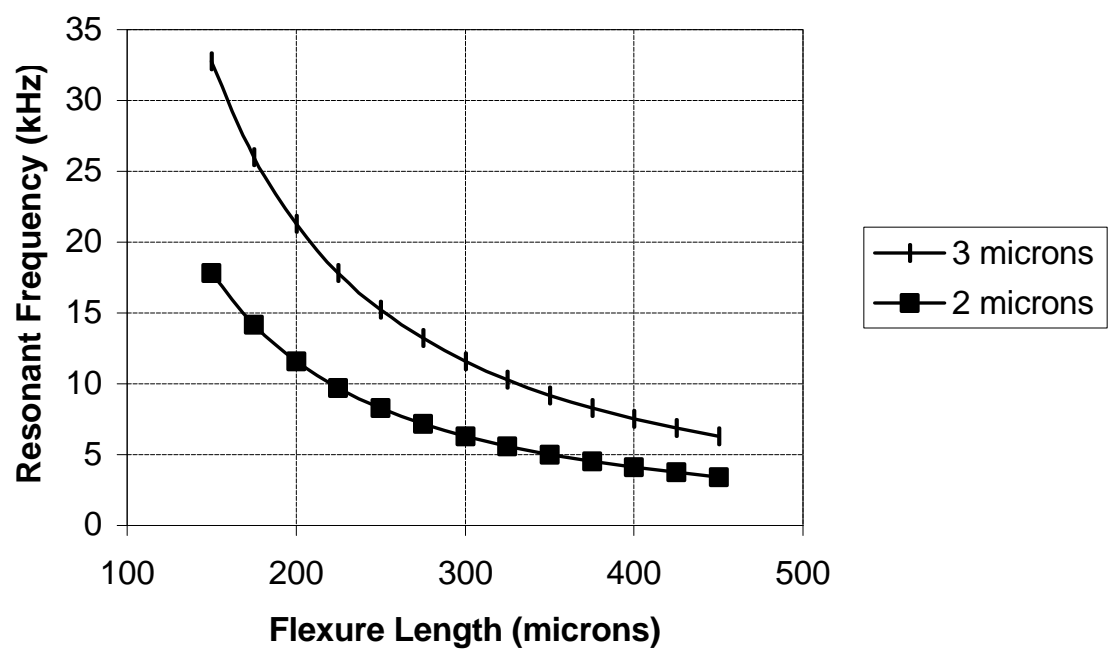

Figure 3.5: Length of Flexure vs. Resonant Frequency - Geometry B

Table 3.3: Flexure and Frequency Data for Geometry B

\begin{tabular}{|c|c|c|}
\hline $\begin{array}{c}\text { Length of } \\
\text { Flexure }(\mu \mathrm{m})\end{array}$ & $\begin{array}{c}\text { Resonant Frequency } \\
(\mathrm{kHz}) \\
3 \mu \mathrm{m} \text { width }\end{array}$ & $\begin{array}{c}\text { Resonant Frequency } \\
(\mathrm{kHz}) \\
2 \mu \mathrm{m} \text { width }\end{array}$ \\
\hline \hline 150 & 32.79 & 17.85 \\
\hline 175 & 26.02 & 14.17 \\
\hline 200 & 21.30 & 11.59 \\
\hline 225 & 17.85 & 9.72 \\
\hline 250 & 15.24 & 8.30 \\
\hline 275 & 13.21 & 7.19 \\
\hline 300 & 11.59 & 6.31 \\
\hline 325 & 10.28 & 5.60 \\
\hline 350 & 9.20 & 5.00 \\
\hline 375 & 8.30 & 4.52 \\
\hline 400 & 7.53 & 4.10 \\
\hline 425 & 6.89 & 3.74 \\
\hline 450 & 6.31 & 3.44 \\
\hline
\end{tabular}




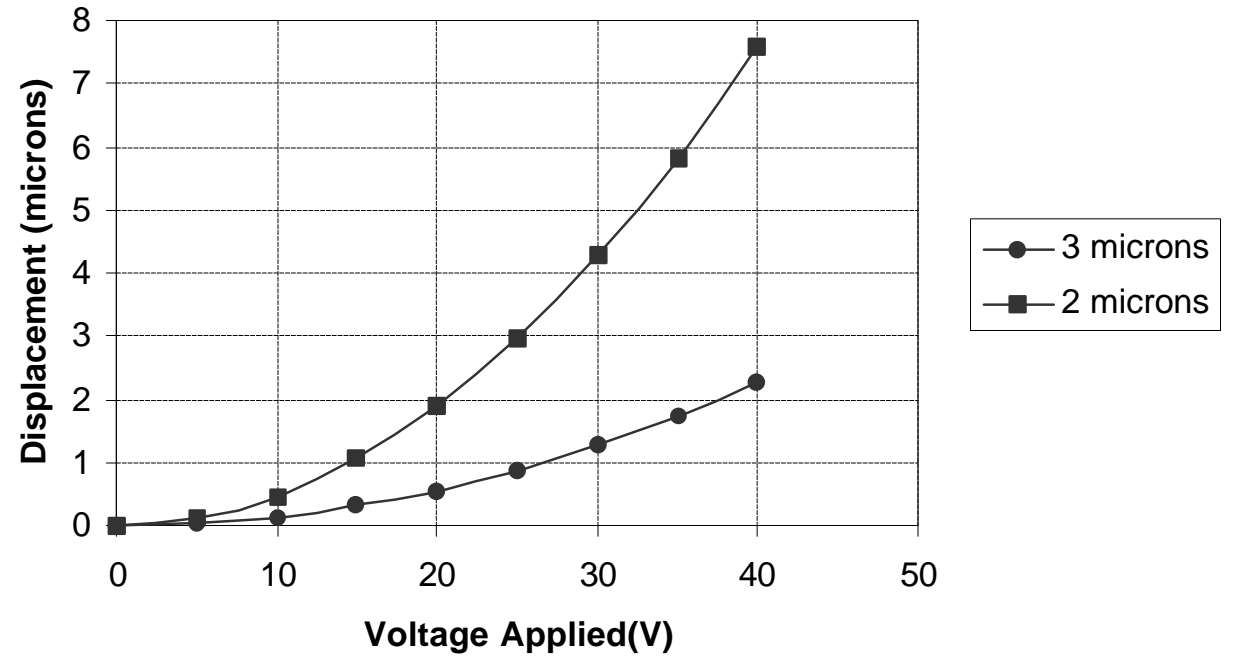

Figure 3.6: Voltage vs. Displacement - Geometry B

Table 3.4: Voltage and Displacement Data with Length $=300 \mu \mathrm{m}$, Geometry B

\begin{tabular}{|c|c|c||}
\hline Voltage $(\mathrm{V})$ & $\begin{array}{c}\text { Displacement }(\mu \mathrm{m}) \\
3 \mu \mathrm{m} \text { width }\end{array}$ & $\begin{array}{c}\text { Displacement }(\mu \mathrm{m}) \\
2 \mu \mathrm{m} \text { width }\end{array}$ \\
\hline \hline 0 & 0 & 0 \\
\hline 5 & 0.035 & 0.119 \\
\hline 10 & 0.141 & 0.475 \\
\hline 15 & 0.316 & 1.068 \\
\hline 20 & 0.526 & 1.898 \\
\hline 25 & 0.879 & 2.966 \\
\hline 30 & 1.265 & 4.271 \\
\hline 35 & 1.722 & 5.813 \\
\hline 40 & 2.250 & 7.592 \\
\hline
\end{tabular}


These results showed that the $2 \mu \mathrm{m}$ width and long flexure lengths would give the desired lateral movement. The next step was to keep the $2 \mu \mathrm{m}$ wide flexure and increase the length even further. Because of the increased flexure length, the number of fingers was doubled to 64 . The comb split was also increased to $150 \mu \mathrm{m}$ to ease waveguide coupling. Both of these changes increased the mass to $1.9563 \times 10^{-10} \mathrm{~kg}$. These devices were labeled Geometry A because they are the most numerous on the die. Figures 3.7 3.9 show the results of varying the length from 350 to $550 \mu \mathrm{m}$. Due to the metrology of the waveguide integration process, a monolithic structure was conceived in order to aid in detecting the lateral motion of the stage. This structure was added as an extra polysilicon layer to the basic 64 finger structures with a 150 waveguide split for flexure lengths from 350 to $550 \mu \mathrm{m}$. 


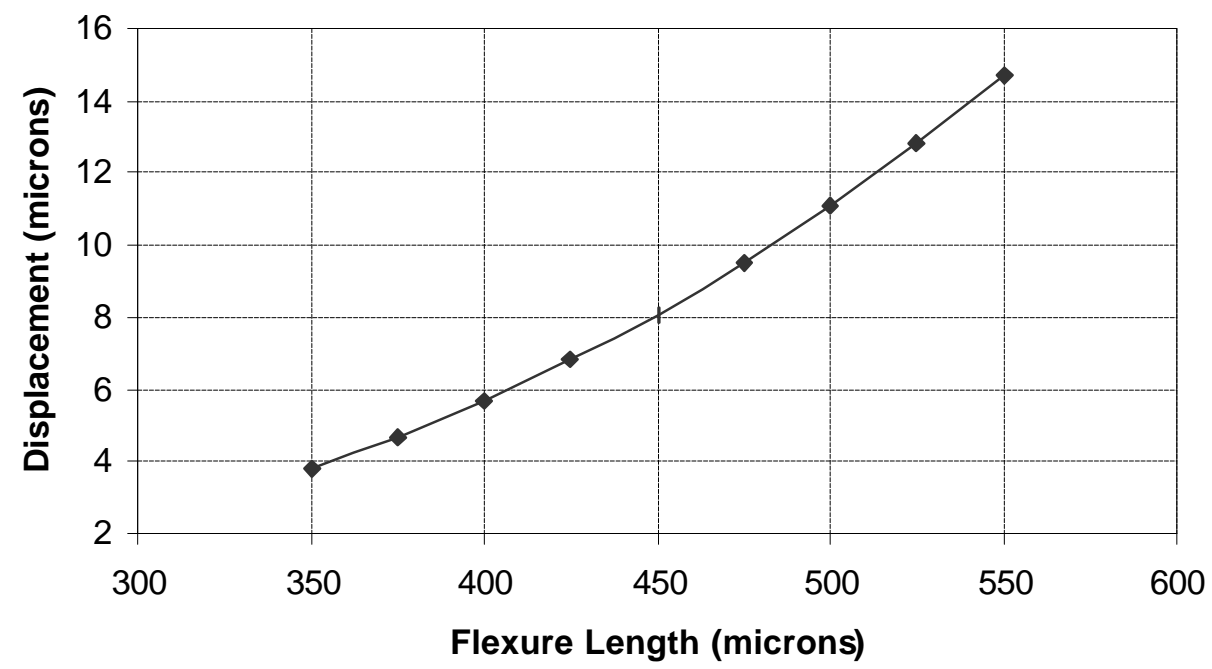

Figure 3.7: Length of Flexure vs. Displacement - Geometry A

Table 3.5: Flexure and Displacement Data with V = 20V, Geometry A

\begin{tabular}{|c|c|}
\hline $\begin{array}{c}\text { Flexure Length } \\
(\mu \mathrm{m})\end{array}$ & $\begin{array}{c}\text { Displacement } \\
(\mu \mathrm{m})\end{array}$ \\
\hline 350 & 3.80 \\
\hline 375 & 4.67 \\
\hline 400 & 5.67 \\
\hline 425 & 6.80 \\
\hline 450 & 8.07 \\
\hline 475 & 9.49 \\
\hline 500 & 11.07 \\
\hline 525 & 12.81 \\
\hline 550 & 14.73 \\
\hline
\end{tabular}




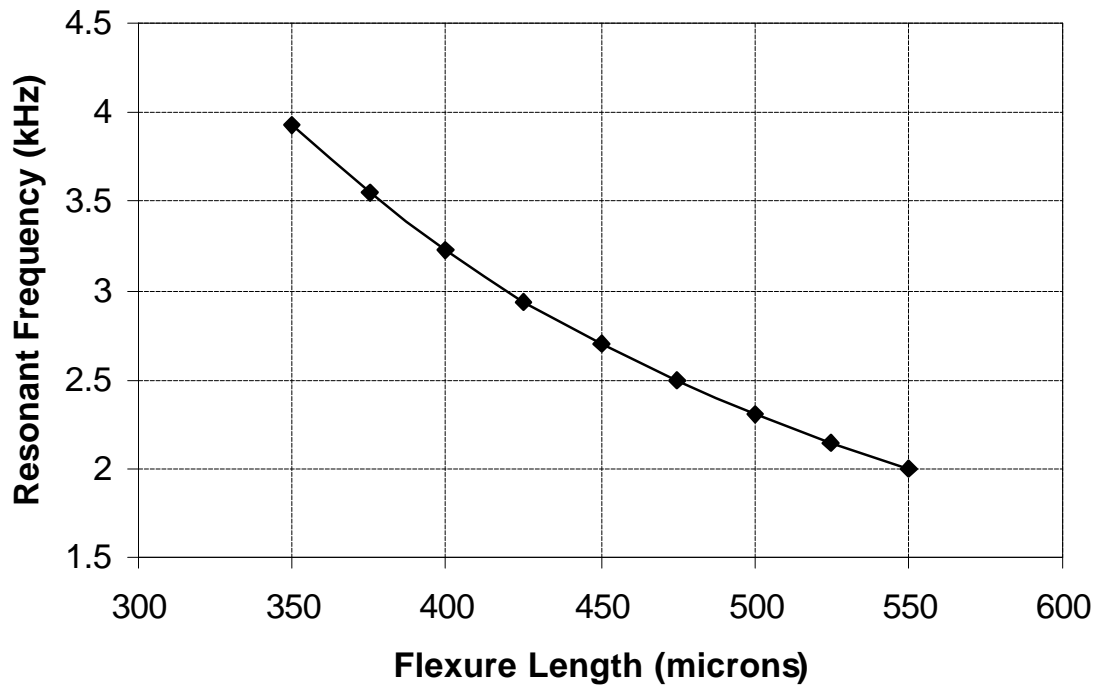

Figure 3.8: Length of Flexure vs. Resonant Frequency - Geometry A

Table 3.6: Flexure and Frequency Data for Geometry A

\begin{tabular}{|c|c|}
\hline $\begin{array}{c}\text { Flexure Length } \\
(\mu \mathrm{m})\end{array}$ & $\begin{array}{c}\text { Resonant Frequency } \\
(\mathrm{kHz})\end{array}$ \\
\hline 350 & 3.93 \\
\hline 375 & 3.55 \\
\hline 400 & 3.22 \\
\hline 425 & 2.94 \\
\hline 450 & 2.7 \\
\hline 475 & 2.49 \\
\hline 500 & 2.3 \\
\hline 525 & 2.14 \\
\hline 550 & 2 \\
\hline
\end{tabular}




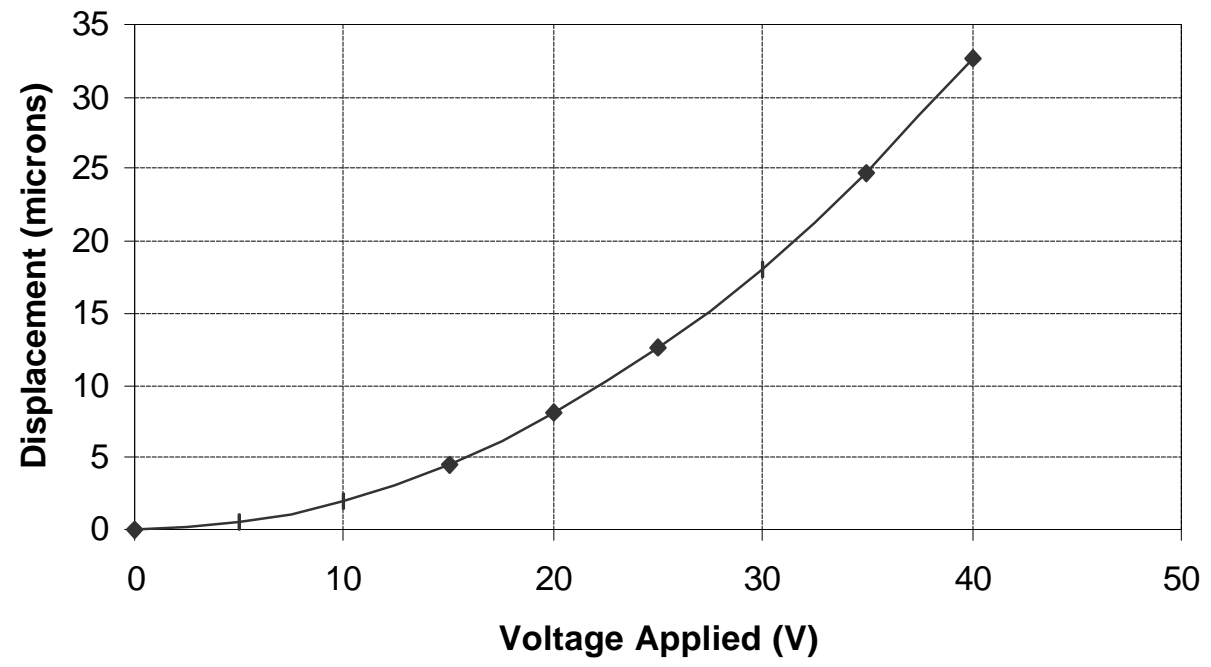

Figure 3.9: Voltage vs. Displacement - Geometry A

Table 3.7: Voltage and Displacement Data with Length $=450 \mu \mathrm{m}$, Width $=2 \mu \mathrm{m}$, Geometry A

\begin{tabular}{|c|c||}
\hline $\begin{array}{c}\text { Voltage } \\
(\mathrm{V})\end{array}$ & $\begin{array}{c}\text { Displacement } \\
(\mu \mathrm{m})\end{array}$ \\
\hline 0 & 0 \\
\hline 5 & 0.50 \\
\hline 10 & 2.02 \\
\hline 15 & 4.54 \\
\hline 20 & 8.07 \\
\hline 25 & 12.55 \\
\hline 30 & 18.11 \\
\hline 35 & 24.76 \\
\hline 40 & 32.59 \\
\hline
\end{tabular}


It was theorized that the fingers might bend away from the voltage field underneath them. To correct this problem, strips of silicon in the dimple layer were included along the length of the fingers in order to make them more rigid. Dimples are structures on the bottom of the stage that reduce stiction caused by moisture that collects under the stage of the device in an open-air environment. They provide a surface for the device to rest on rather than directly on the substrate, and are small enough not to cause any significant friction. Because of the fabrication process design constraints, the finger width had to be increased to $10 \mu \mathrm{m}$ to incorporate the dimples. This reduced the number of fingers back to 32 while maintaining the same geometry of the center stage, but further increased the mass to $2.8724 \times 10^{-10} \mathrm{~kg}$. These figures were labeled Geometry C. Figures $3.10-3.12$ show the results of this arrangement. 


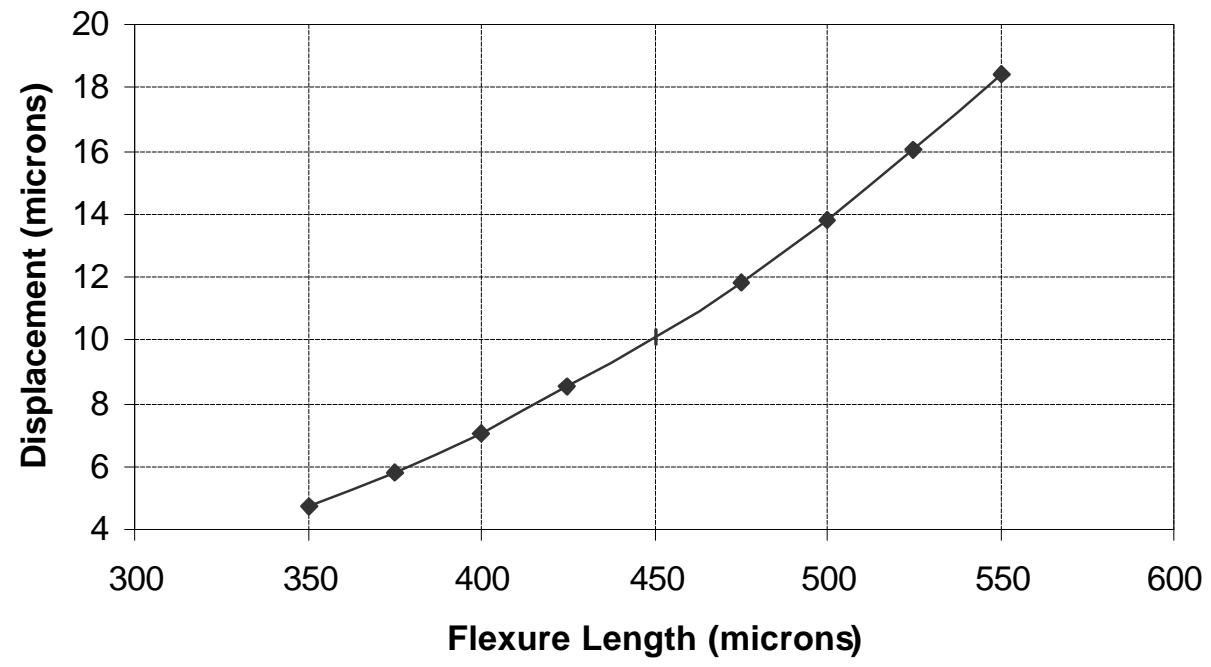

Figure 3.10: Flexure Length vs. Displacement - Geometry C

Table 3.8: Flexure and Displacement Data with V = 20V, Geometry C

\begin{tabular}{|c|c|}
\hline $\begin{array}{c}\text { Flexure Length } \\
(\mu \mathrm{m})\end{array}$ & $\begin{array}{c}\text { Displacement } \\
(\mu \mathrm{m})\end{array}$ \\
\hline 350 & 4.75 \\
\hline 375 & 5.84 \\
\hline 400 & 7.08 \\
\hline 425 & 8.50 \\
\hline 450 & 10.09 \\
\hline 475 & 11.86 \\
\hline 500 & 13.83 \\
\hline 525 & 16.02 \\
\hline 550 & 18.41 \\
\hline
\end{tabular}




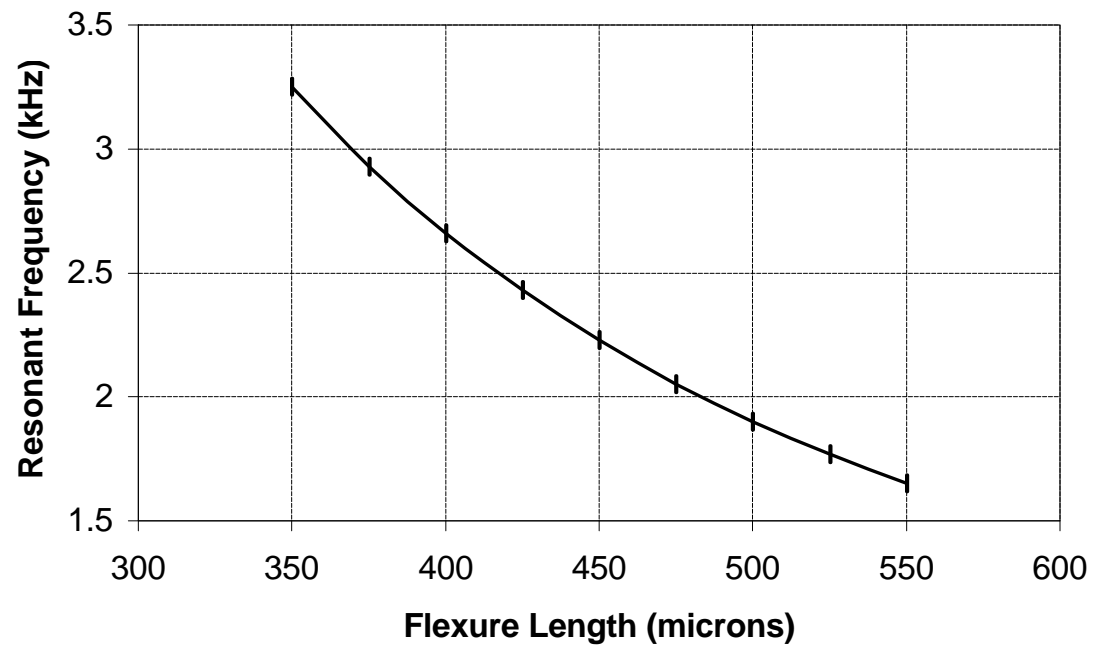

Figure 3.11: Length of Flexure vs. Resonant Frequency - Geometry C

Table 3.9: Flexure and Frequency Data for Geometry C

\begin{tabular}{|c|c|}
\hline $\begin{array}{c}\text { Flexure Length } \\
(\mu \mathrm{m})\end{array}$ & $\begin{array}{c}\text { Resonant Frequency } \\
(\mathrm{kHz})\end{array}$ \\
\hline 350 & 3.25 \\
\hline 375 & 2.93 \\
\hline 400 & 2.66 \\
\hline 425 & 2.43 \\
\hline 450 & 2.23 \\
\hline 475 & 2.05 \\
\hline 500 & 1.9 \\
\hline 525 & 1.77 \\
\hline 550 & 1.65 \\
\hline
\end{tabular}




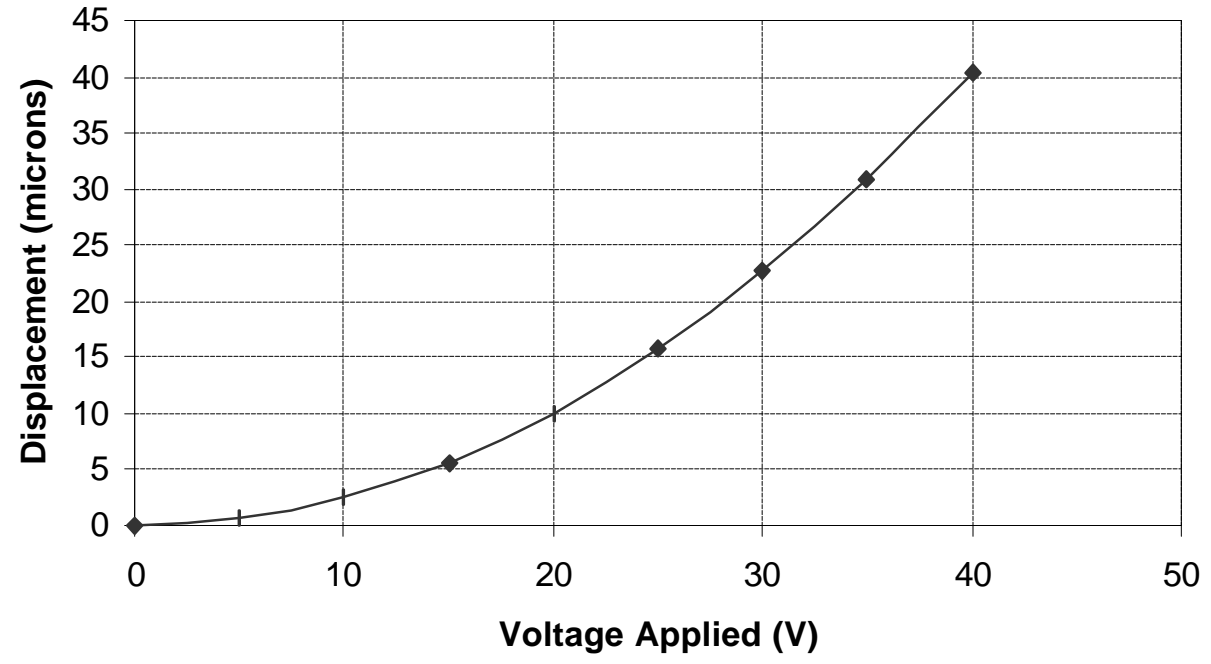

Figure 3.12: Voltage vs. Displacement - Geometry C

Table 3.10: Voltage and Displacement Data with Length $=450 \mu \mathrm{m}$, Width $=2 \mu \mathrm{m}$, Geometry C

\begin{tabular}{|c|c||}
\hline $\begin{array}{c}\text { Voltage } \\
(\mathrm{V})\end{array}$ & $\begin{array}{c}\text { Displacement } \\
(\mu \mathrm{m})\end{array}$ \\
\hline \hline 0 & 0 \\
\hline 5 & 0.63 \\
\hline 10 & 2.52 \\
\hline 15 & 5.67 \\
\hline 20 & 10.09 \\
\hline 25 & 15.76 \\
\hline 30 & 22.69 \\
\hline 35 & 30.89 \\
\hline 40 & 40.34 \\
\hline
\end{tabular}


Table 3.11 Device Parameters

\begin{tabular}{|c|c|c|c|}
\hline MUMPS 20 & Mass (kg) & Number of Fingers & Data Plots \\
\hline \hline Geometry A & $1.956 \times 10^{-10}$ & 64 & $3.3-3.6$ \\
\hline Geometry B & $1.206 \times 10^{-10}$ & 64 & $3.7-3.9$ \\
\hline Geometry C & $2.872 \times 10^{-10}$ & 32 & $3.10-3.12$ \\
\hline
\end{tabular}

\subsection{Device Layout}

The MEMS structures were previously drawn on LASI, a mask drawing software. The mask layouts were then sent to the Microelectronics Center of North Carolina (MCNC) for use in device fabrication. The layout of the device structures is shown in Figure 3.13. Geometry A with the added monolithic structure can be observed in Figure 3.13 from the right of center, and the monolith can be viewed in Figure 3.14. To the left of center in Figure 3.13 are the same structures without the monolith. The arrangement is shown closer in Figure 3.15 as Geometry A. The comb drives with the $50 \mu \mathrm{m}$ split (Geometry B) are located to the far right of Figure 3.13, with flexure lengths varying from 300 to $450 \mu \mathrm{m}$. Other than the split, they have the same geometry as Geometry A. Figure 3.16 shows Geometry B with the smaller comb split. Figure 3.17 illustrates the comb drive with reinforced dimpled fingers (Geometry C), with a close up in Figure 3.18. They are located to the far left in Figure 3.13 and have flexure lengths of $500 \& 550 \mu \mathrm{m}$. To aid in the actual testing of these devices, text was included in the first layer of polysilicon to ease in the identification of the flexure length and number of fingers. This is shown in Figure 3.19. 


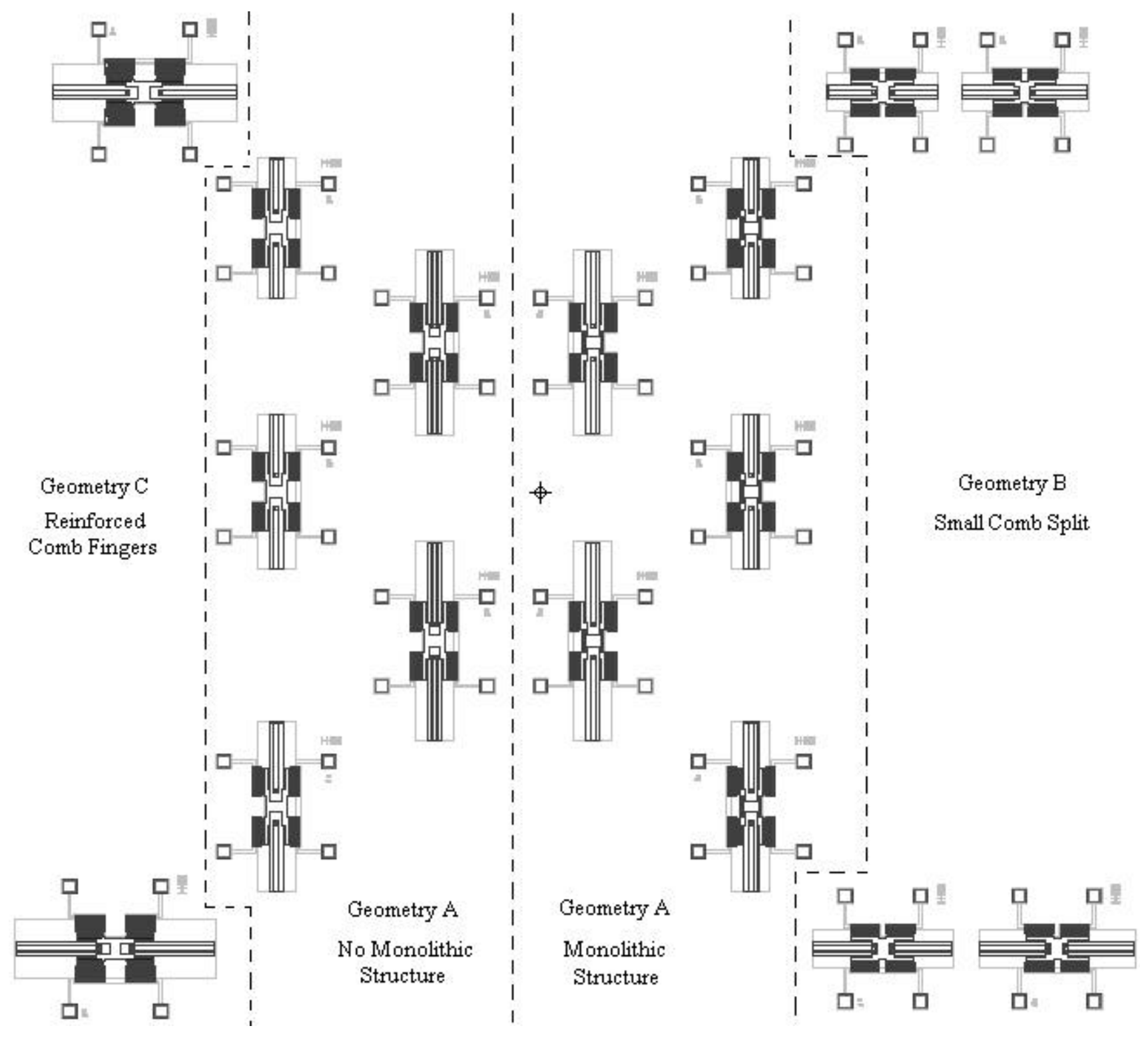

Figure 3.13: Device Layout on $1 \mathrm{~cm}$ x $1 \mathrm{~cm}$ Die 


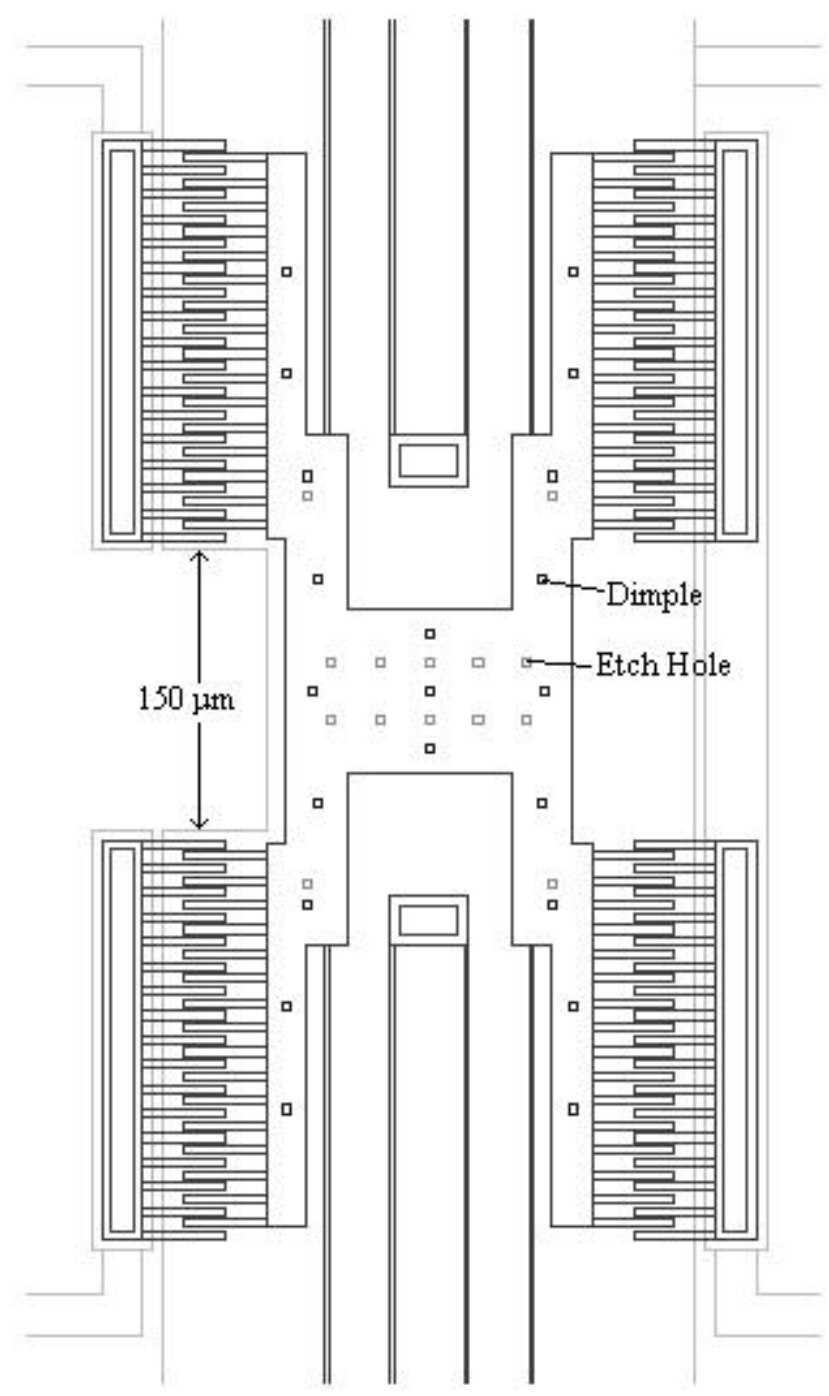

Figure 3.14: Geometry A 


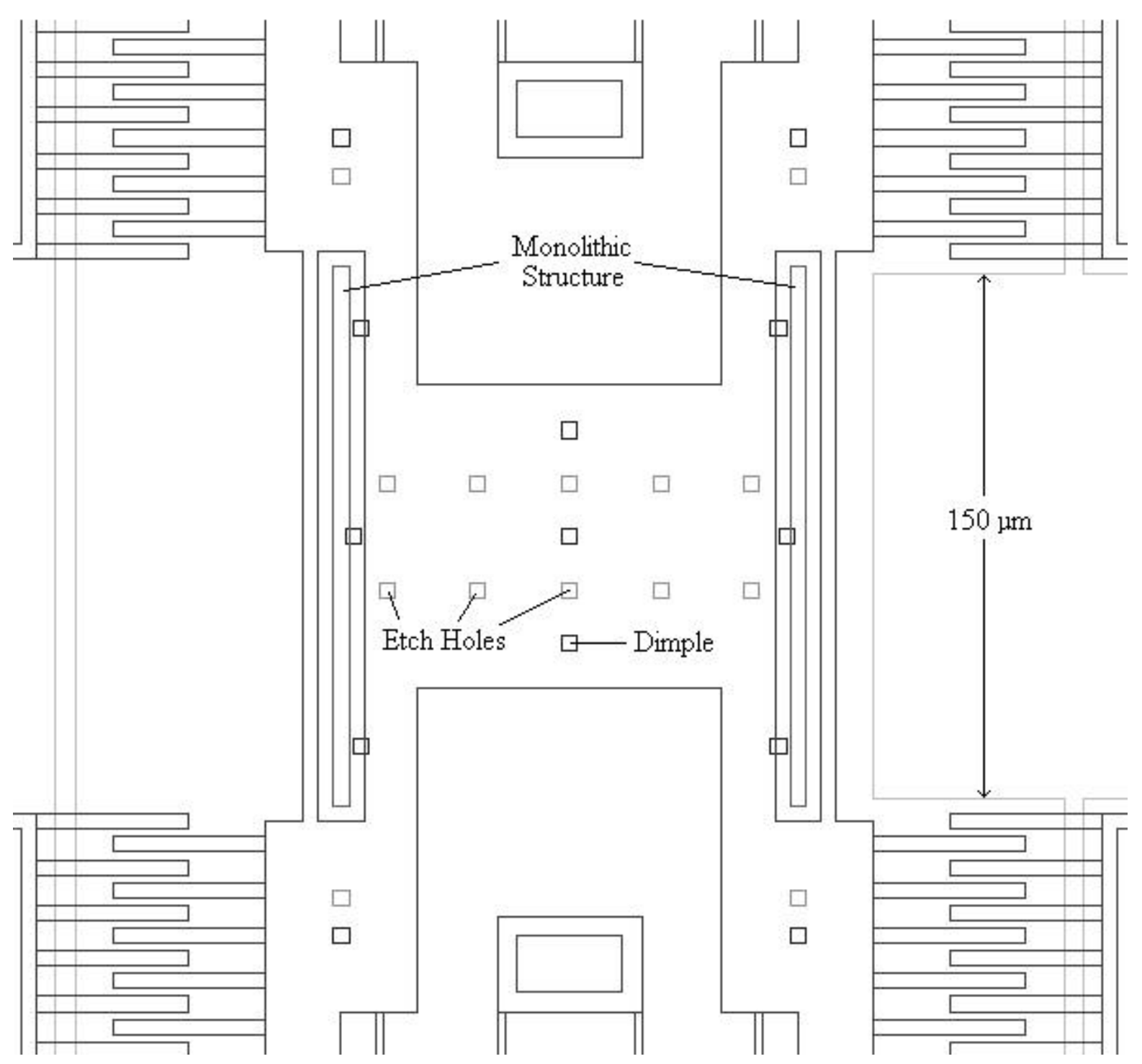

Figure 3.15: Close-up of Monolithic Structure 


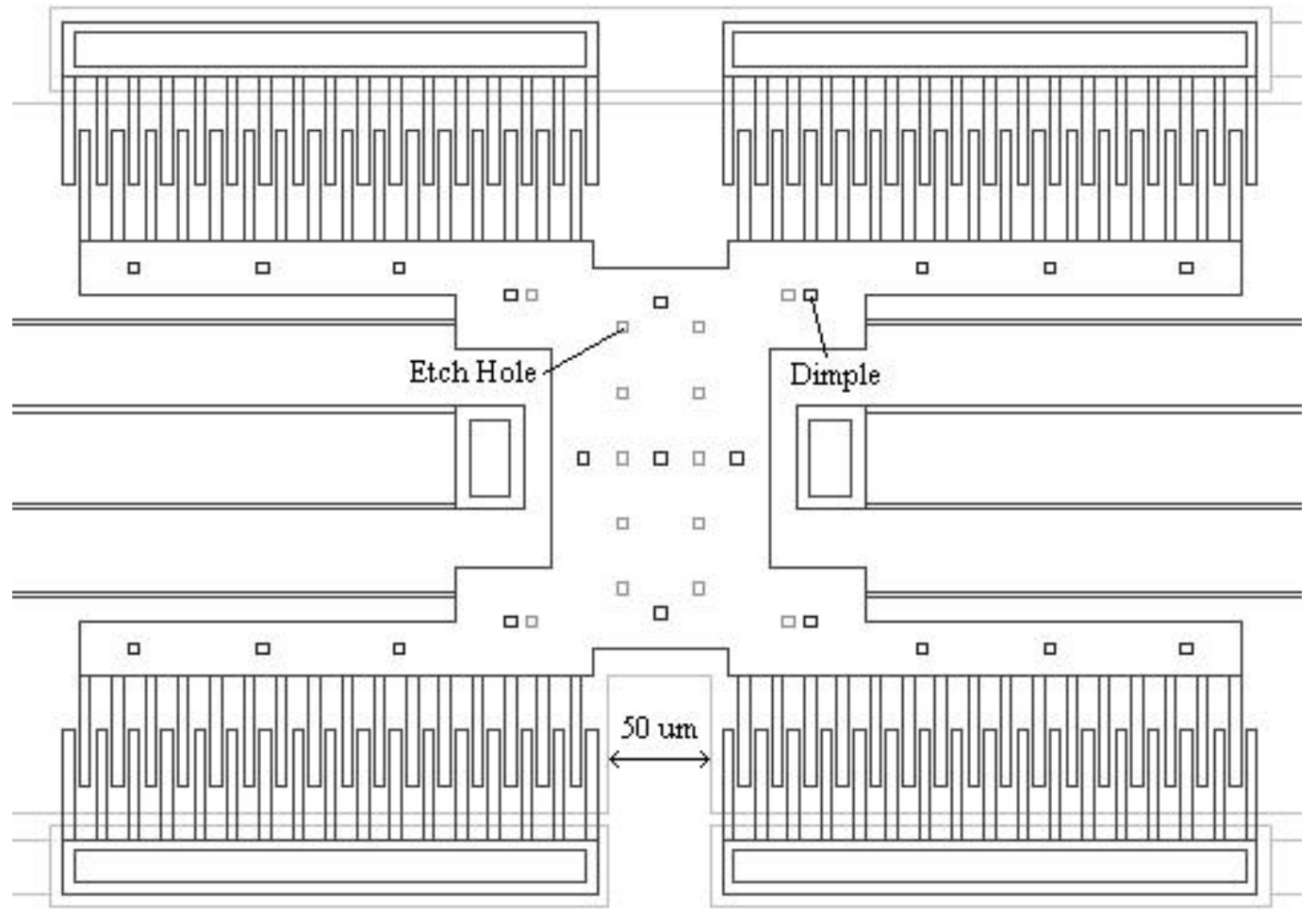

Figure 3.16: Geometry B 


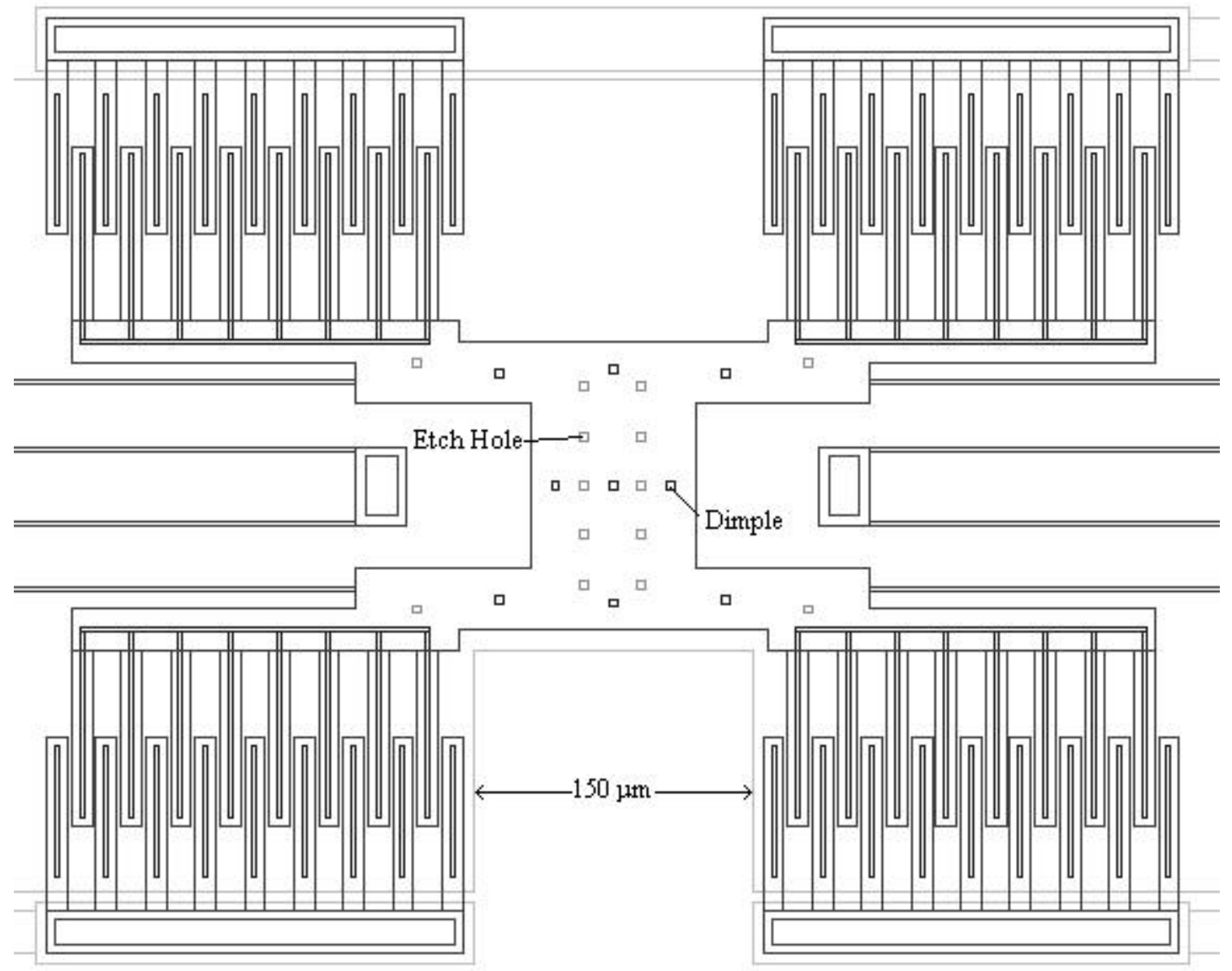

Figure 3.17: Geometry C 


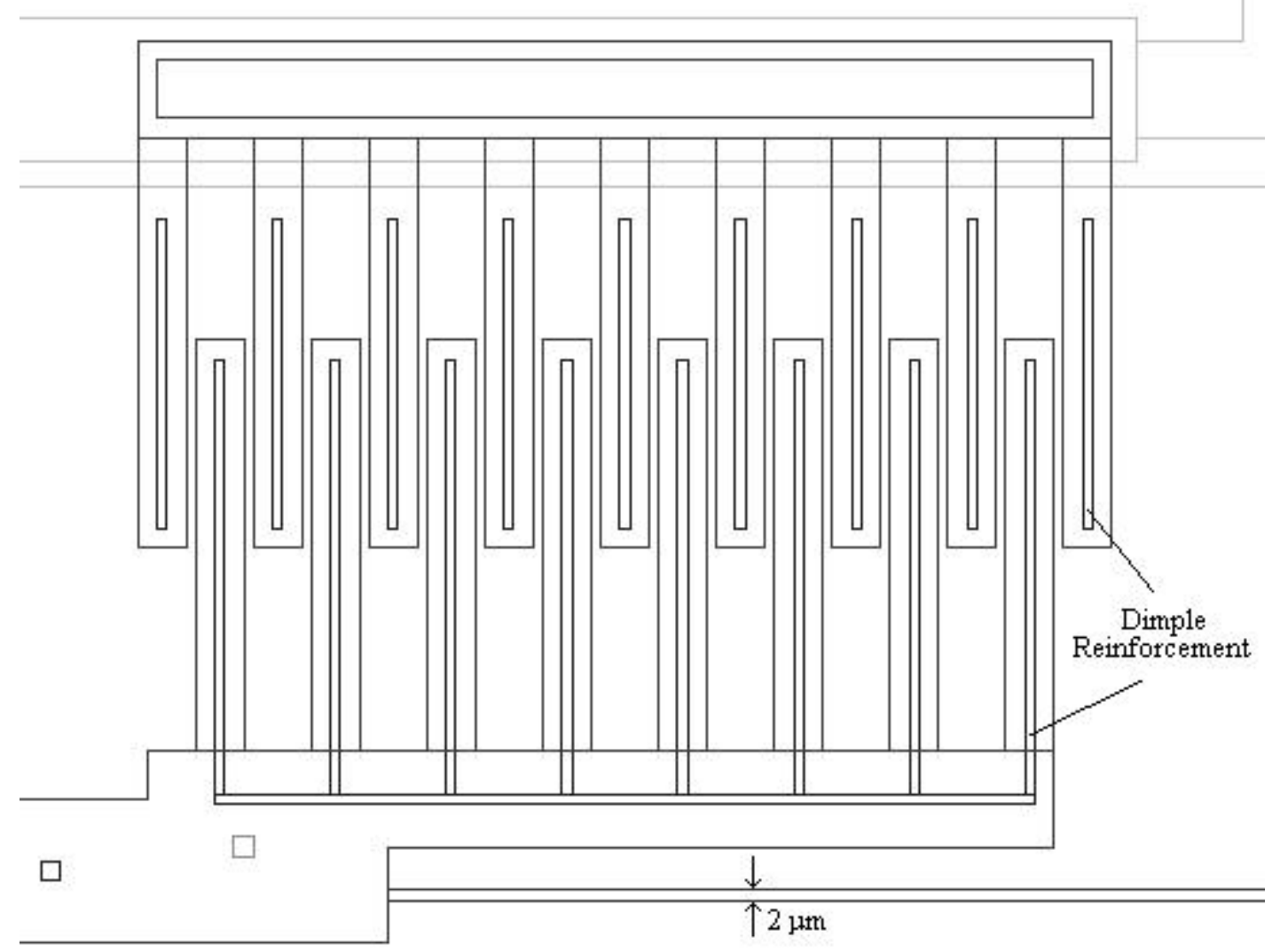

Figure 3.18: Close-up of Reinforced Comb Fingers 


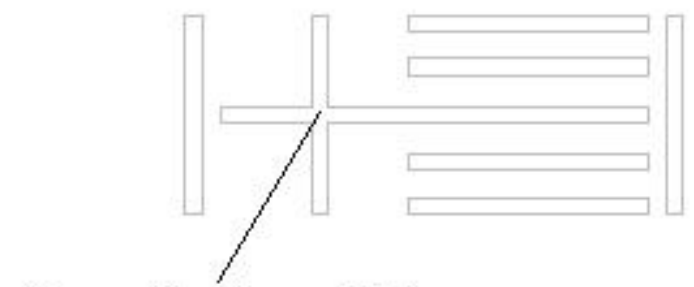

Waveguide Alignment Mark
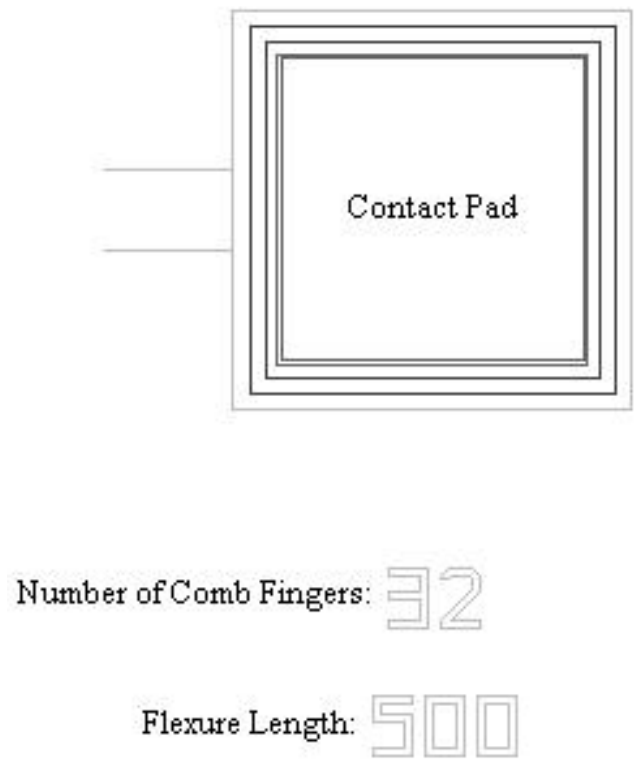

Figure 3.19: Device Identification and Alignment Marks 


\subsection{Release Process}

The layout in figure 3.13 was sent to MCNC for fabrication and the finished dice were returned in three months. Their Multi-User MEMS Process (MUMPs), outlined in Appendix B, provides 16 dice per die location purchased. The dice are shipped with a photoresist coating on the front side to protect the structures from any damage that may occur during shipping. To remove this coating, the die must be soaked in acetone with ultrasonic agitation for 20 minutes. The die is then placed in isopropyl alcohol for one minute and rinsed thoroughly with de-ionized (DI) water.

The structures must be released, the term given to the removal of sacrificial oxide layers between polysilicon layers, in order to allow movement of the translation stage. To do this the die is placed in a room temperature bath of $49 \%$ hydrofluoric acid (HF) for 2.0 to 2.5 minutes with gentle agitation. This is followed by a 10 minute rinse in DI water and 5 minutes in isopropyl alcohol. The die is then baked for 15 to 30 minutes to remove any excess liquid that would cause stiction. If the devices do not operate properly, this process is repeated to remove any unremoved oxide under the moving parts.

\subsection{Through-Wafer Utilization}

Even though the design of the Phase III devices was primarily for waveguide cointegration, certain aspects were well suited for the application of through-wafer interconnections. The silicon substrate is transparent to $1.3 \mu \mathrm{m}$ (near infrared) light. The split comb design allowed an uncluttered region for the beam to interact with the translation stage. Due to the small thicknesses of the layers of the device, infrared light 
passing through the translation stage would not have encountered enough attenuation to cause a decrease in the output signal. The inclusion of monolithic structures on some of the devices solved this problem so that a totally new design did not have to be created. Geometry A with the monolithic structures included was the only geometry utilized in this research.

\subsection{Backside Wafer Polishing}

The backside of the MEMS device die was initially unpolished so any beam sent through it would experience a drastic decrease in intensity due to of scattering before it interacted with stage of the device. Because of this, a Cambridge Instruments METROPOL 2 rotational sample polisher was used to smooth the backside of the wafer die [39]. Before release, the die was mounted on the polishing chuck using brown wax. The chuck was placed on a polishing pad coated with a 6-micron diamond suspension and left to rotate for 1-1.5 hours. After the majority of the surface was smoothed, the pad was changed and covered with a 1-micron suspension and allowed to spin for 15-20 minutes. The next step involved cleaning the wax and stripping the photoresist. This involved a 20 minute soak in acetone, followed by a 5 minute soak in methanol, followed by a 5 minute rinse in DI water. If this process wasn't sufficient to remove all of the wax, the process was repeated with a 5 minute soak in acetone with ultrasound, another 5 minutes in methanol, completed by another 5 minute rinse in DI water. In one case this still was not sufficient to remove all of the wax, so the process was repeated after a first step of a 5 minute soak in acetone heated to $40^{\circ} \mathrm{C}$. 


\section{Chapter 4}

\section{Through-Wafer Testing}

This chapter presents the modeling and characterization of the infrared optical transmission of a silicon MUMPS die and the effect on this transmission by a MEMS

device fabricated on the die surface. Expected transmission percentage is calculated using the multilayer transmission topics covered in Chapter 2, along with the determination of spot size on the surface of the wafer. The experimental optical probe setup needed to input and measure the beam intensity is described in detail. Image capture results with a stationary translation stage, used to evaluate the feasibility of the work, are presented. Optical power measurements with the stage stationary and electrostatically driven are also shown, along with the optical characterization of device motion and resonance frequency.

\subsection{Expected Transmission}

To implement a through-Si wafer method of monitoring, a wavelength of light must be used for which the silicon substrate is transparent. The bandgap of silicon (1.12 $\mathrm{eV}$ ) is such that it is transparent to infrared light at a wavelength of $\sim 1300 \mathrm{~nm}$. The 
intensity of a transmitted beam of this wavelength should be decreased by the presence of the moving device stage interrupting it when it is directed through the substrate from below. The following figure shows a simplified view of the device layers of the MEMS die:

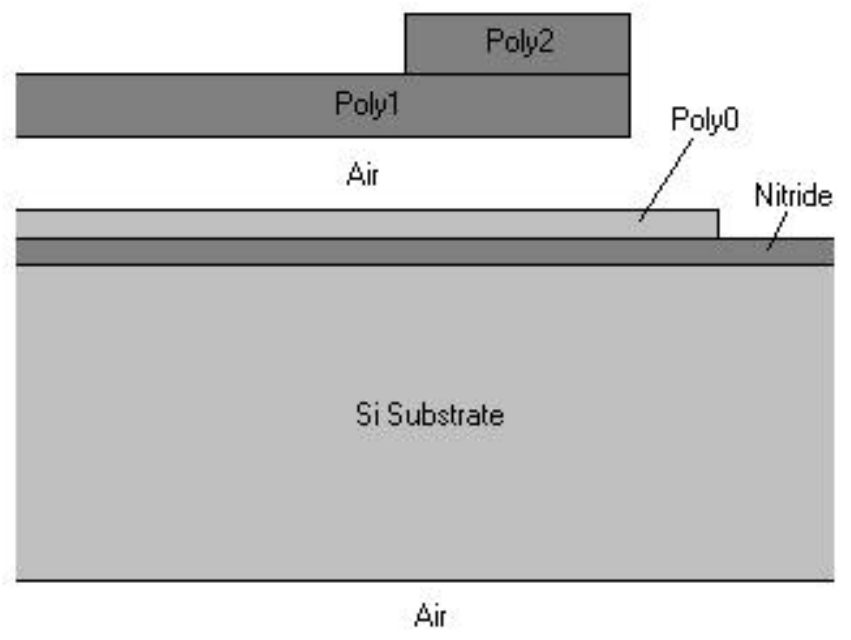

Figure 4.1: Device Layers

The silicon substrate and polysilicon layers all have an index of refraction $n=3.5$, the LPCVD deposited nitride layer was chosen to have a nominal value of $n=2$, and $n=1$ for air. The first interface the infrared beam encounters is the air-substrate interface on the bottom side of the die. The reflectance of this interface is given by equation (2.2-1) where $n_{1}$ is the index of refraction of air and $n_{2}$ is the index of the substrate. This gives a reflectance of 30.9\% from equation (2.2-2). The transmittance has a value of $69.1 \%$ of the input intensity, obtained from equation (2.2-3).

In order to improve this value, an antireflective coating of nitride could be deposited on the bottom surface of the die. The thickness of this layer can be determined 
by plotting reflectance versus nitride thickness. Using the equation for the coefficient $\beta$ (eq. 2.2-5) containing $h$ varied from 0 to $1.0 \mu \mathrm{m}$, and substituting into the equation for the reflectance of a film between two media (eq. 2.2-8) gives the plot shown in Figure 4.2.

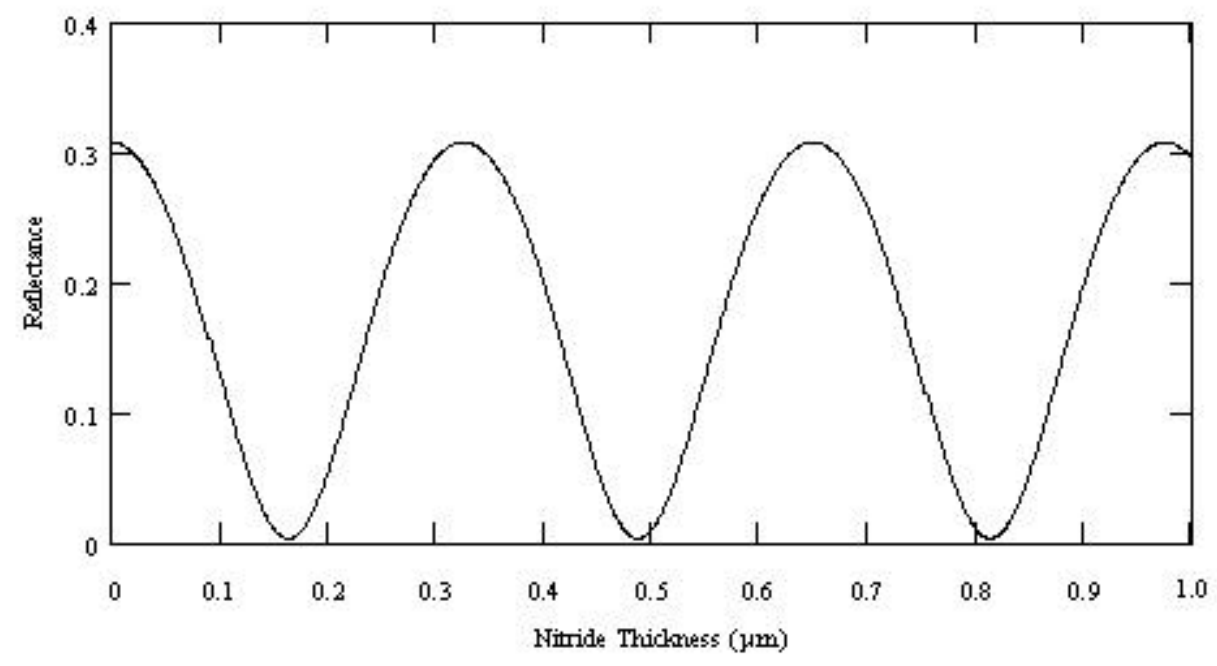

Figure 4.2: Reflectance as a Function of Nitride Thickness

It can be observed from this plot that minimum reflectance occurs at every multiple of quarter-wavelengths in the medium. Using the characteristic matrix in (2) with the thickness, $h$, in (3) as $0.5 \mu \mathrm{m}$ decreases the reflectance to 0.011 , giving a transmittance into the silicon substrate of 0.989 . These figures were obtained assuming negligible absorption losses in the media with $\lambda_{0}=1.3 \mu \mathrm{m}$, and a coherent light source.

This method can be repeated for the other interfaces in the device. The top substrate-nitride-air interface is shown to have a transmittance of 0.74 . This is the fraction of the intensity observed by the infrared detector when the beam is uninterrupted by the stage of the device. The layers of polysilicon on the translation stage have a 
transmittance of only 0.294 . The combination of these transmittances gives $74 \%$ through POLY 0, 70\% through POLY0/air/POLY1, and 21\% through these layers plus POLY 2. With the infrared source powered with a DC signal, the total modulation depth when transmission through all layers is read by the IR detector should be $21 \%$ of the signal read through unpatterned silicon.

\subsection{Experimental Setup}

In order to apply the needed combination of light sources, focusing optics, and detectors, an experimental setup had to be devised that would allow the infrared beam to be focused and manipulated to achieve the highest signal throughput possible. It would also have to accommodate the positioning of probes that would provide the means to energize the devices for simulated in-situ measurements. The following diagrams show an overall view of the entire testing system and the basic experimental setup surrounding the MEMS device. 


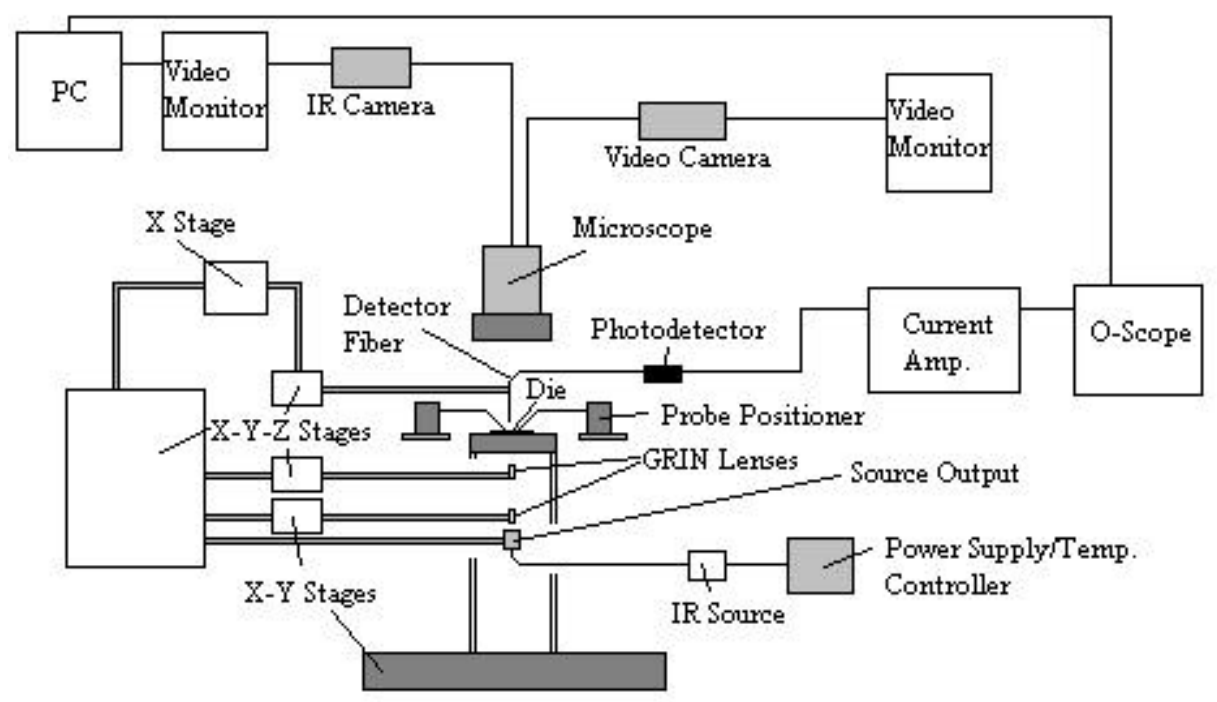

Figure 4.3 Experimental Setup

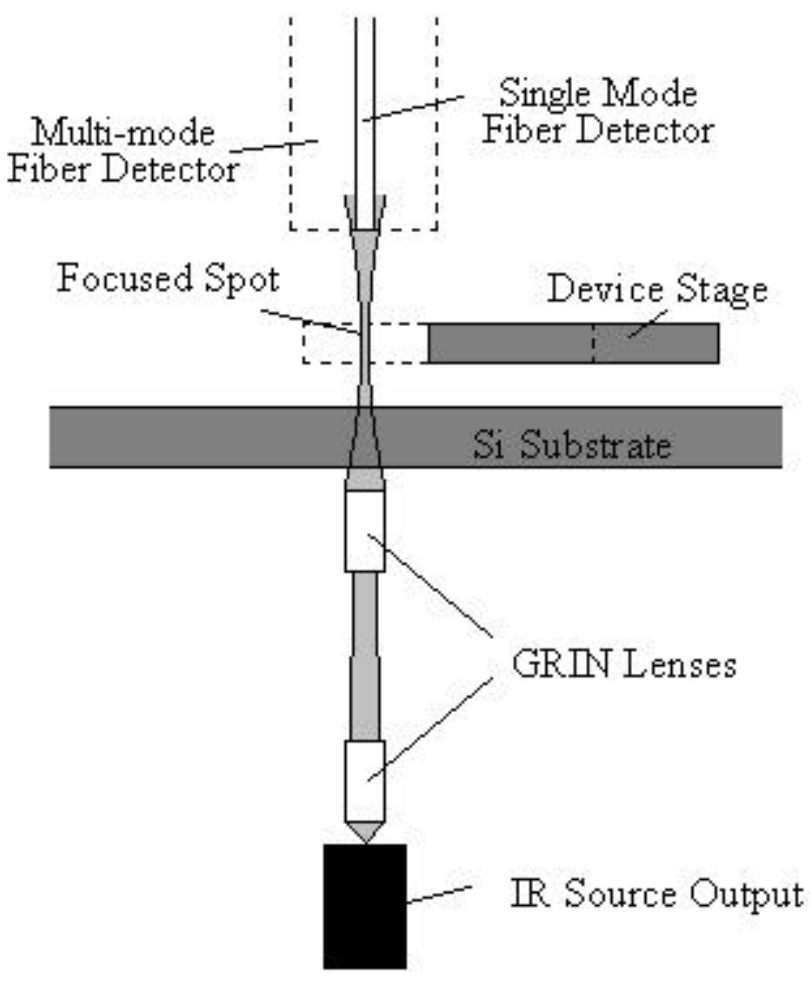

Figure 4.4: Representation of Beam Interaction with Device Stage 
The light source and GRIN lenses used to focus the IR beam are situated under the die and are both attached to an $x-y-z$ stage, with the top GRIN lens on a separate $x-y-z$ stage for increased adjustment. The bottom GRIN lens, when used, was only movable in the xy plane. The MEMS die itself is located on a piece of aluminum with a $1 \mathrm{~cm}$ hole drilled in it to allow the beam to pass through the die and be probed as well. The platform is resting on the $\mathrm{x}-\mathrm{y}$ stage of the probe station to allow for adjustment of the devices on the die when positioning probes or performing stationary device stage experiments. The probes are magnetically attached to the probe platforms of the station and are $\mathrm{x}-\mathrm{y}-\mathrm{z}$ adjustable as well. Attached to the same $x-y-z$ stage as the light source output and GRIN lenses is an apparatus that holds the optical fiber coupled to the infrared detector. It has its own $x-y-z$ adjustment as well for increased coupling of the beam. When positioning probes or performing image capture tests, the fiber holder can be moved completely out of the field of view of the microscope using the x-stage so the devices can be viewed. Attached to the microscope is an infrared camera with outputs to a video monitor and an image capture card, as well as a standard camera with regular video output.

\subsection{Infrared Light Sources/Detectors}

\subsubsection{LED}

Two different infrared sources were used to perform through-wafer measurements. The first was an LED previously characterized at Bell Laboratories [40]. It emitted incoherent light at $1300 \mathrm{~nm}$ with an angular divergence of $\sim 20^{\circ}$ from a $1 \times 12$ array of $62.5 \mu \mathrm{m}$ core multi-mode fibers that were connected to the emitting device by an 
optical fiber ribbon cable with a polished silicon V-groove end face. The LED powered by pin 6 was used in all through-wafer tests performed with this device.

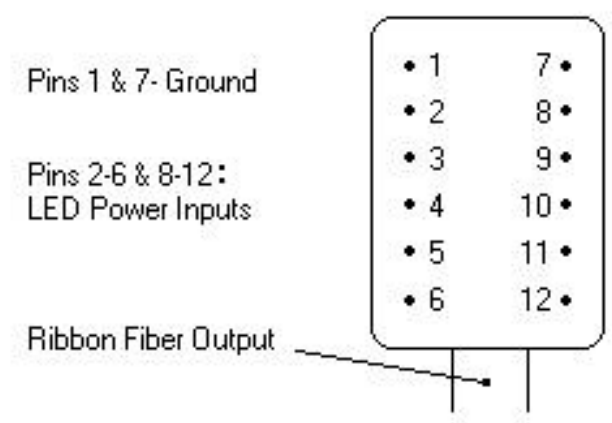

Figure 4.5: LED Pin Configuration

Measured by the InGaAS detector, maximum output intensity of $\sim 0.1 \mu \mathrm{W}$ was observed through unpatterned silicon with input currents in the range of 150-175 mA supplied from an HP 6128B power supply. An HP 8013A pulse generator was used for pulsed LED outputs.

\subsubsection{Laser Diode}

Due to the low output power of the LED, and infrared laser diode was obtained to improve through-wafer transmission intensities. The laser diode was a fiber pigtailed Newport model LD-1310-31B that emitted coherent monochromatic (1 nm FWHM) light at a wavelength of $1310 \mathrm{~nm}$ with a threshold current of $6 \mathrm{~mA}$. The pigtail was a $9 \mu \mathrm{m}$ single mode fiber. The pinouts for this device are shown in Figure 4.6. 


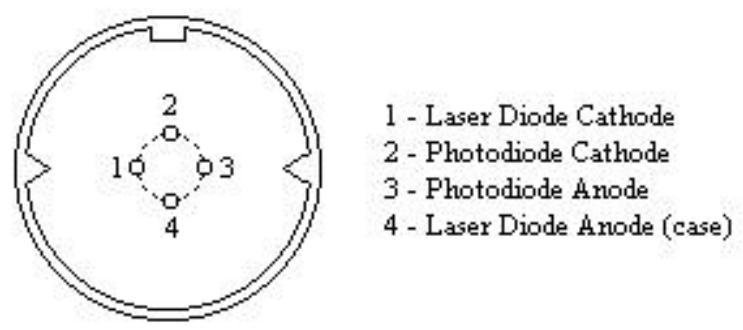

Figure 4.6: Laser Diode Pin Configuration

The diode was mounted on an ILX Lightwave LDT-5910 temperature controller that kept the diode at $20^{\circ} \mathrm{C}$, and power was delivered to pins $1 \& 4$ from an ILX Lightwave LDX3620 ultra low noise current source. For modulated output, an HP 8111A pulse/function generator was input to the current source set on DC modulation. The increase in output power caused the spot to flood the IR camera, so input currents only ranged from 5-10 $\mathrm{mA}$ in order for the spot to be viewed. Output power in this range of current was $\sim 100$ $\mu \mathrm{W}$. Maximum allowable input current to the device was $20 \mathrm{~mA}$.

\subsubsection{Germanium Detector}

The first IR photodetector used was a germanium detector from United Detector Technologies. At $1300 \mathrm{~nm}$ its specifications state that it has a response of $0.6228 \mathrm{~A} / \mathrm{W}$ with a quantum efficiency of $59.4 \%$. Because of the low power emitted by the LED and the low conversion of the detector, the output from this device had to be amplified by a Stanford Research Systems SR570 low noise current preamplifier. Even with output amplification this detector was unable to read any change in signal when the spot was scanned under the features on the device stage. 


\subsubsection{InGaAs Detector}

The final addition to the experimental setup that, when combined with a laser diode source, allowed for an easily observable through-wafer signal was a FEMTO PR$\mathrm{X}$-10K-IN-DC indium gallium arsenide low noise photoreceiver. This device was powered by with $\pm 15 \mathrm{~V}$ from a Tektronix CPS250 triple output power supply. The sensitivity of this device was $0.95 \mathrm{~A} / \mathrm{W}$ at $1300 \mathrm{~nm}$. With a transimpedance of $1 \times 10^{7} \mathrm{~A} / \mathrm{V}$ this gives a conversion gain of $9.5 \times 10^{6} \mathrm{~V} / \mathrm{W}$, considerably higher than that of the germanium detector. This device had a maximum frequency response of $100 \mathrm{kHz}$. Because of the high output of this device, no current amplification was required.

\subsection{GRIN Lens Applications}

Because of the angular divergence of the IR source outputs, a SELFOC wide numerical aperture, 1/4-pitch, $2 \mathrm{~mm}$ diameter GRIN lens designed for $630 \mathrm{~nm}$ operation was used to focus the beam to decrease the spot size on the top surface of the die. To determine the correct location of object and image distances to achieve the smallest possible spot sizes, equations $2.3-9,2.3-13,2.3-17$, and 2.3-18 were plotted as a function of object distance (Figures 4.7-4.10). 


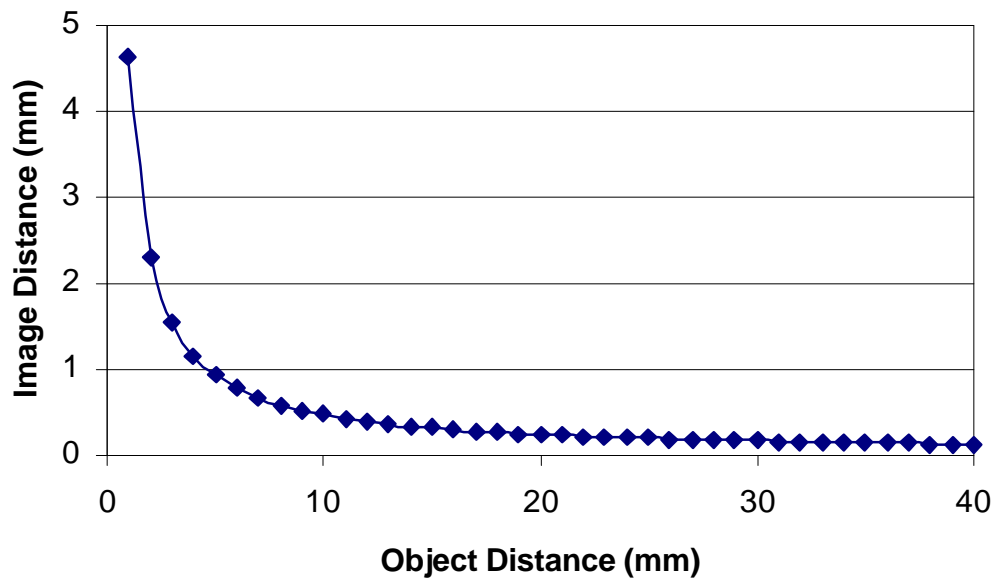

Figure 4.7: Object vs. Image Distances

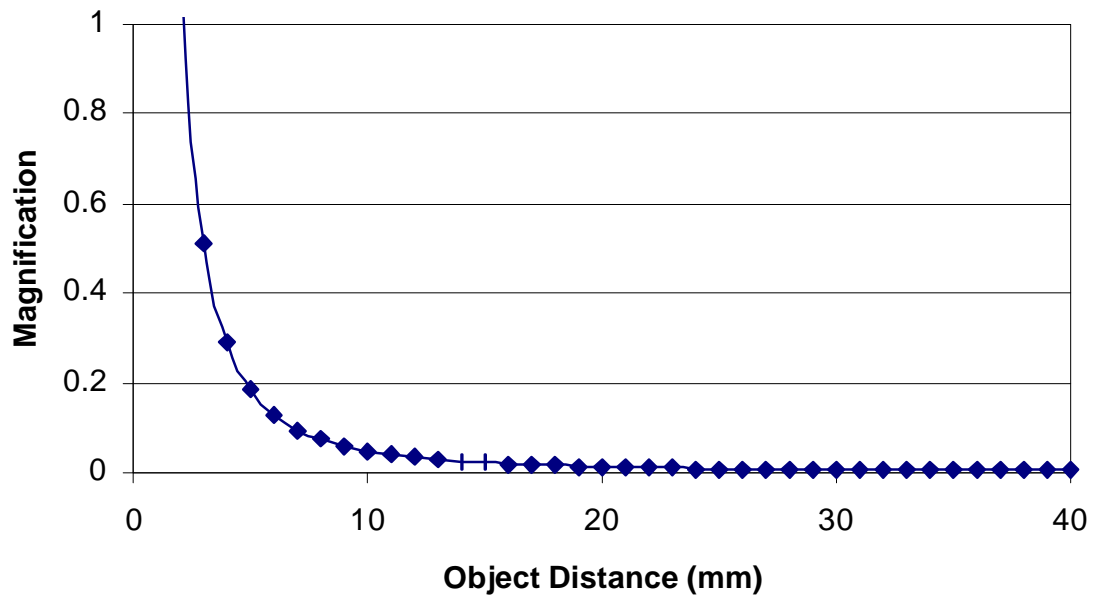

Figure 4.8: Object Distance vs. Magnification 


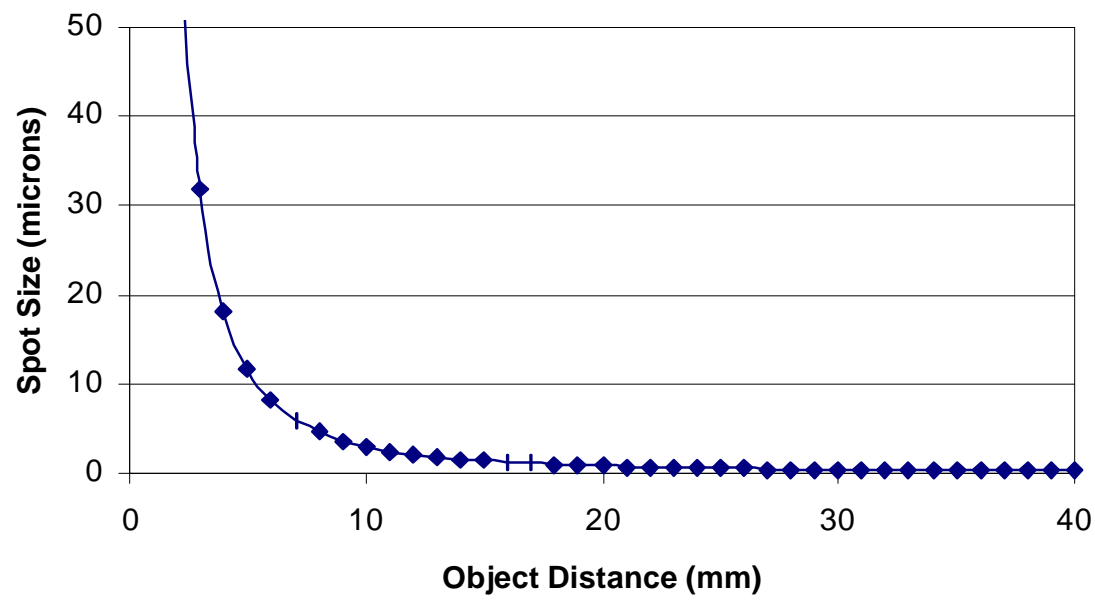

Figure 4.9: Object Distance vs. Spot Size

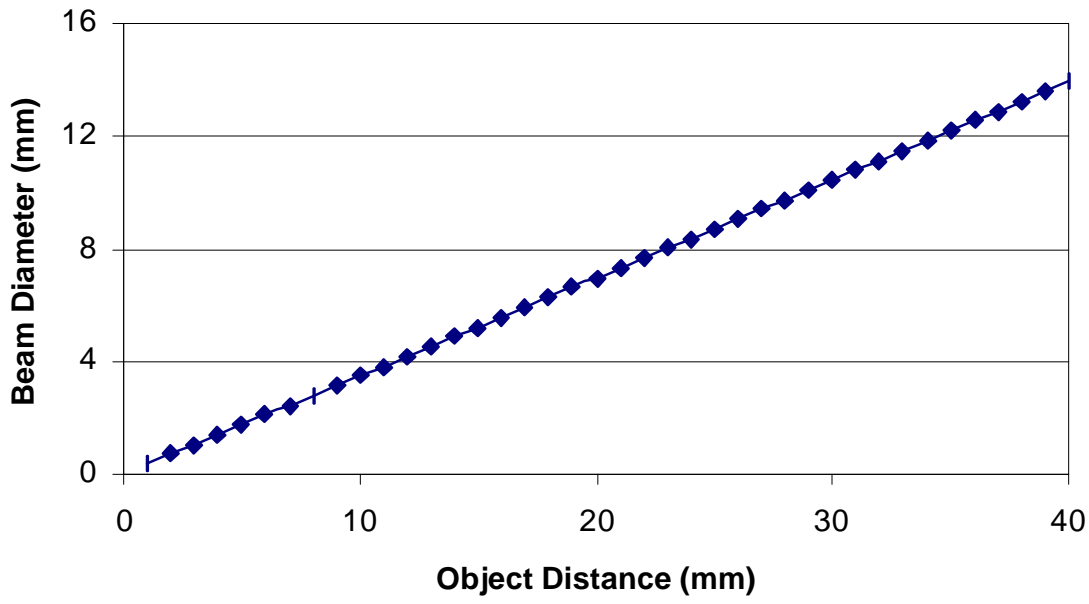

Figure 4.10: Object Distance vs. Beam Diameter at Lens 
For a single GRIN lens setup the source should be located as far away from the lens as possible to get a small spot size, but this also increases the beam diameter (Figure 4.10) and decreases intensity. To achieve smaller spot sizes without a large decrease on intensity, two GRIN lenses were used. One lens collimated the source by placing the IR output at the location found by Equation 2.3-14, effectively making it an infinite distance away form the second lens. The collimated output was then directed through the second lens and focused at the same distance as the source from the first lens. Since the collimated beam acts as a source a relatively large distance away (5-15 mm), this should give spot sizes on the order of 5-20 microns in diameter (Figure 4.8). While giving smaller spot sizes, this dual lens setup will experience more loss due to lens separation and off axis displacement [36].

\subsection{Image Capture Results}

The first step in determining whether the through-wafer methodology would be feasible was to simply observe the intensity of the beam as it was moved under a MEMS device on the die. This initial evaluation was performed using the LED, powered by 150 mA DC, and a single GRIN lens. The spot was displayed on a video monitor attached to the infrared camera, and on a computer with a video capture card. Spot size was determined to be $\sim 50$ microns by comparing it to features on the MEMS device. Existing programs, written in MacRAIL [41], which scanned a region of pixels and listed their grayscale intensity values were modified to scan the area where the spot would interact in actual device motion testing. This involved moving the spot, capturing the image, running the program, and tabulating the data. The spot was then moved to the next 
sequential location, and the process was repeated until the spot had moved the desired distance under the device or feature. To obtain a clear reading, any lighting used to see the stage was eliminated so only the spot was visible in the camera. Figure 4.11 shows five different locations where the image capture was performed under the stage and Figure 4.12 shows three locations that were observed under or near the flexures.

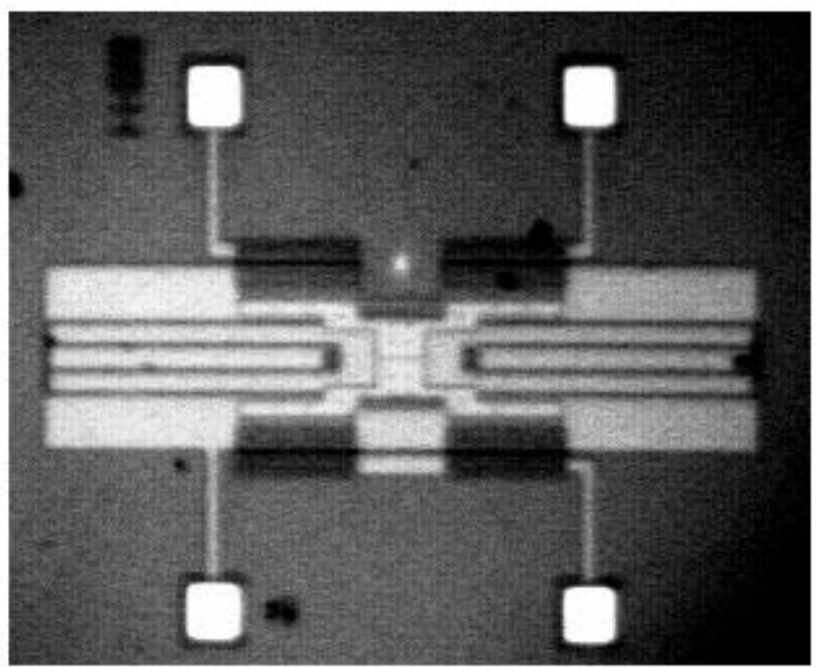

Position 1

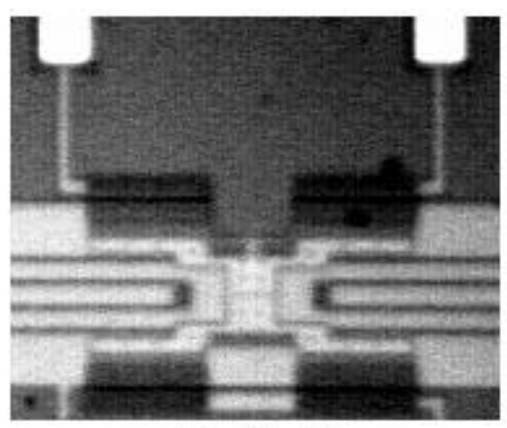

Position 3

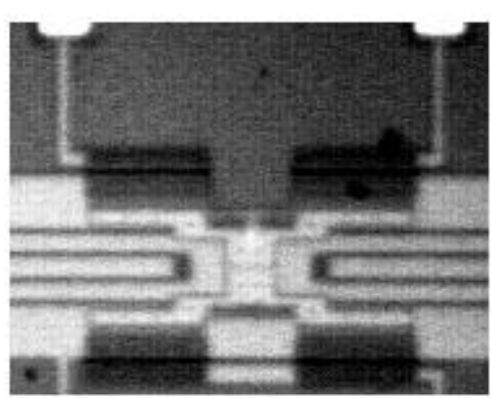

Position 4

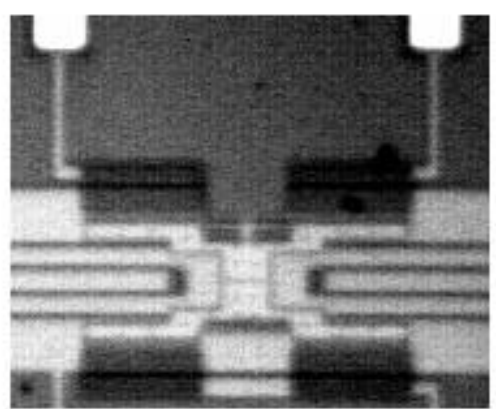

Position 2

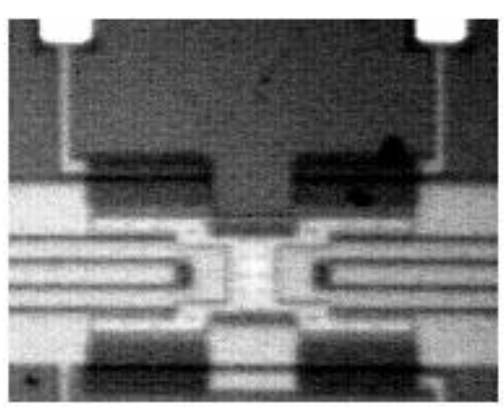

Position 5

Figure 4.11: Spot Progression Under Device Stage (Phase II device) 


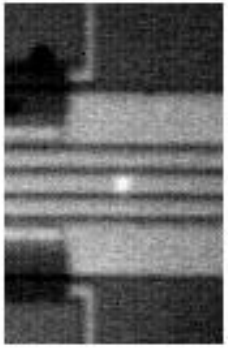

Position 1

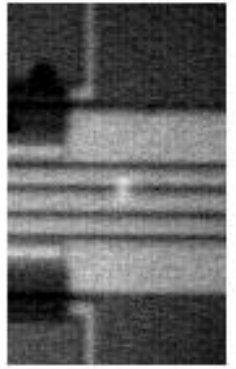

Position 2

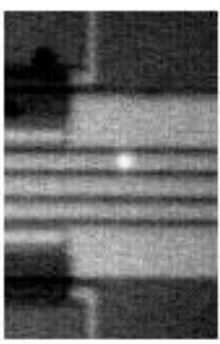

Position 3

Figure 4.12: Spot Progression Under Flexures

At each location, a cross-sectional scan of the spot was taken and grayscale intensities were assigned to each pixel in the row. Figure 4.13 shows the plot of grayscale values and pixel number for the stage progression, and Figure 4.14 shows the plot for the flexure progression.

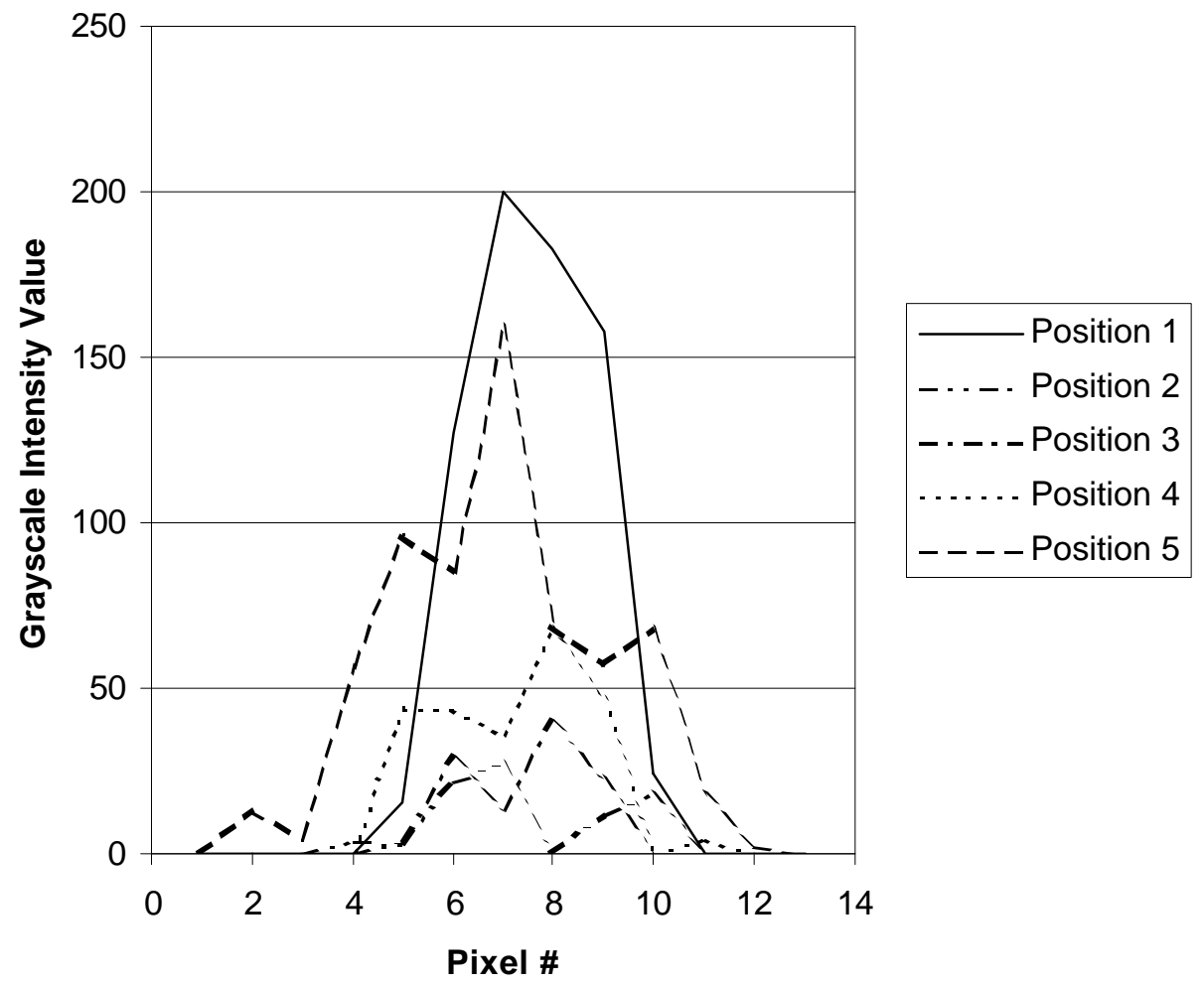

Figure 4.13: Through-Wafer Spot Intensity Under Translation Stage 


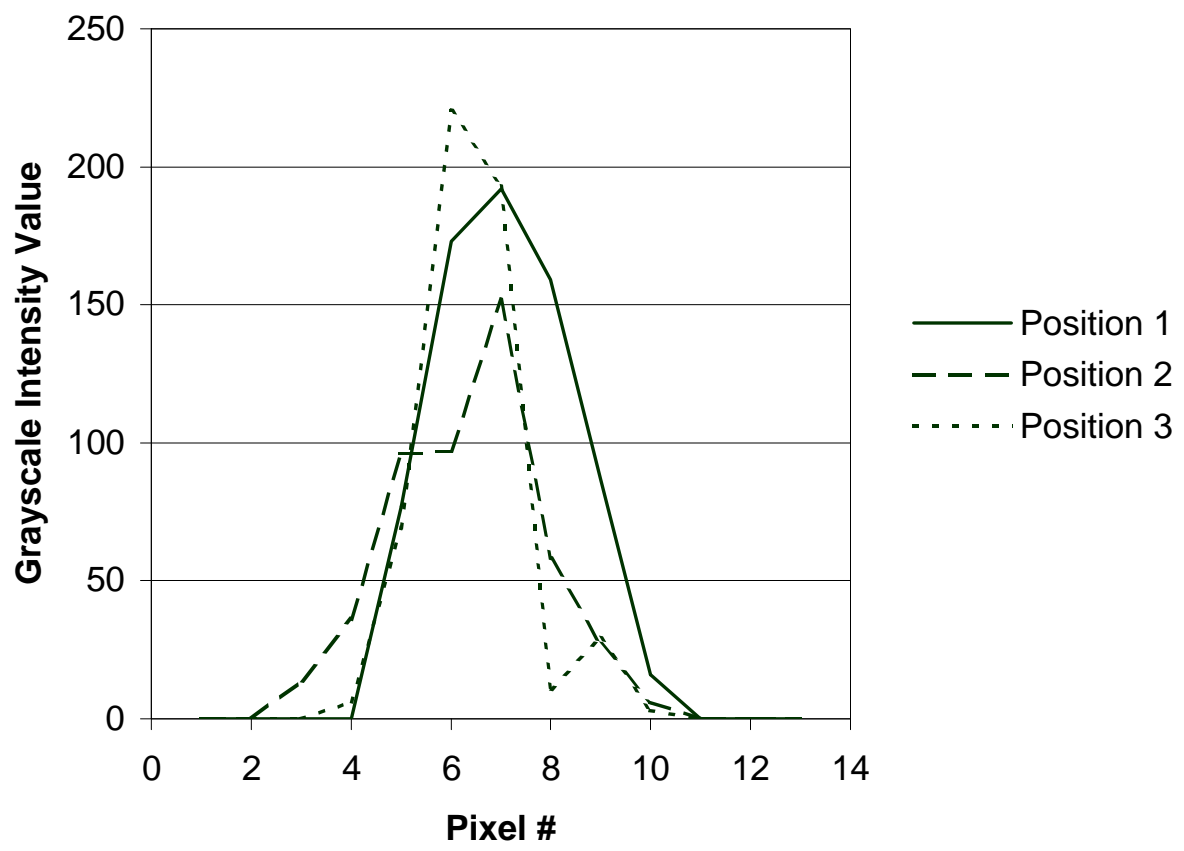

Figure 4.14: Through-Wafer Spot Intensity Under Flexure

To observe how the spot intensity changed as it moved under the stage more accurately, more data points had to be taken. The spot was moved in increments of 0.5 mils (12.7 microns), and the image capture grayscale intensity assignment was done for the pixels in the row for each location. The grayscale intensities in each row were added to give a total intensity value of the spot at each location. It can be observed from Figure 4.15 that there is a definite decrease in the intensity of the spot as it was moved under the stage. It is at its lowest point when the spot is under the strip of POLY 2 fabricated on the stage (monolithic structure) at the location of approximately 63 microns. Lack of resolution in the previous figures is a result of the large spot size of the LED output. 


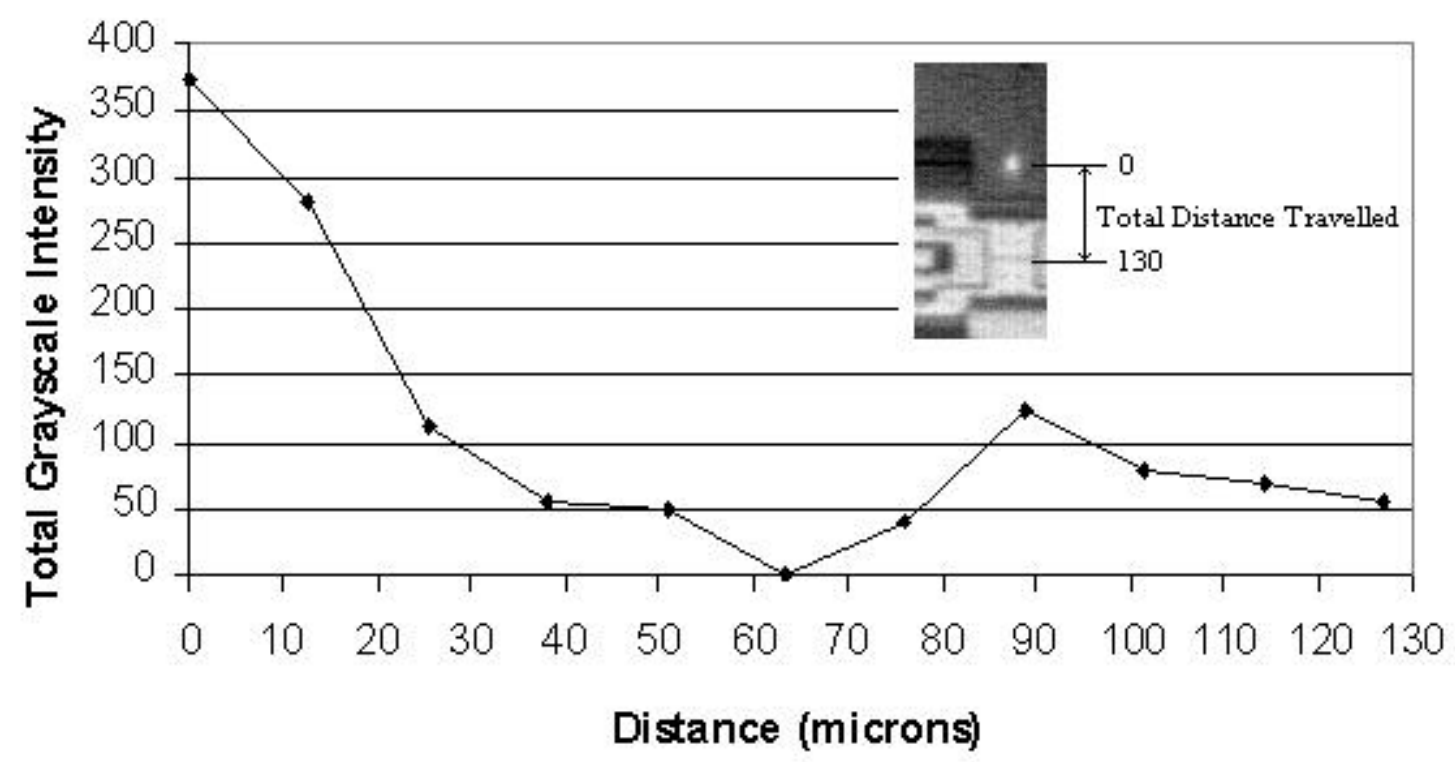

Figure 4.15: Total Intensity as a Function of Distance

\subsection{Power Measurement}

In order to verify the theoretical transmission results discussed in section 4.1 and determine the actual signal caused by the MEMS features, the output of the beam coupled through the MUMPS die was measured with a photodetector. The voltage output given by the detector could then be changed into through-wafer power by using the detector's conversion gain. A laser diode with a small spot size was used to simulate those typical of a diffractive optical system. Unlike the image capture tests where the output was viewed by the camera, these tests used an optical fiber to couple output near the spot focused on the device to the InGaAs detector.

To verify the shape of the output data, a convolution was done using MATLAB that simulated scanning a $25 \mu \mathrm{m}$ spot under a structure with the transmission characteristics of the stage with the strip of polysilicon fabricated on it. This spot size was 
chosen as a worst case value, and no diffraction effects were considered. Figure 4.16 shows the Gaussian beam function, 4.17 the transmission function of the stage, and 4.18 their convolution. This output figure will also change with the core diameter of the detector fiber.

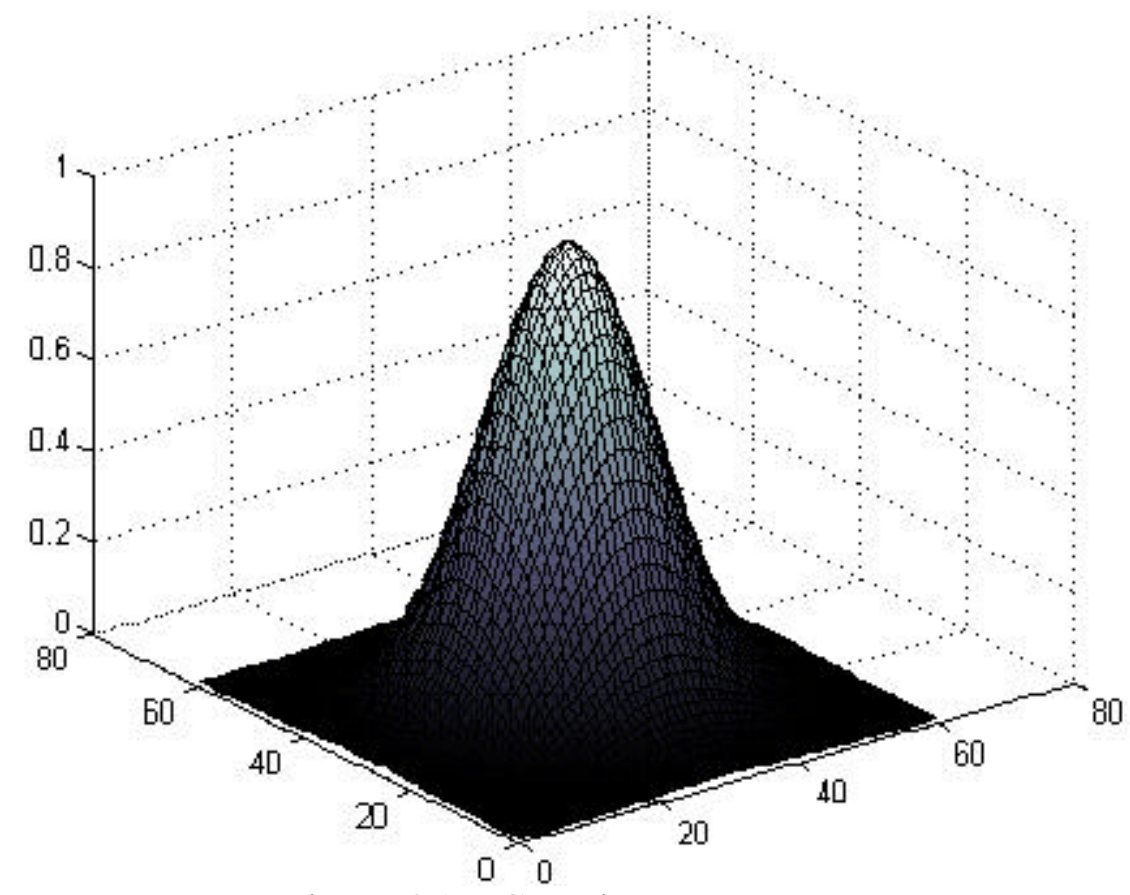

Figure 4.16: Gaussian Beam 


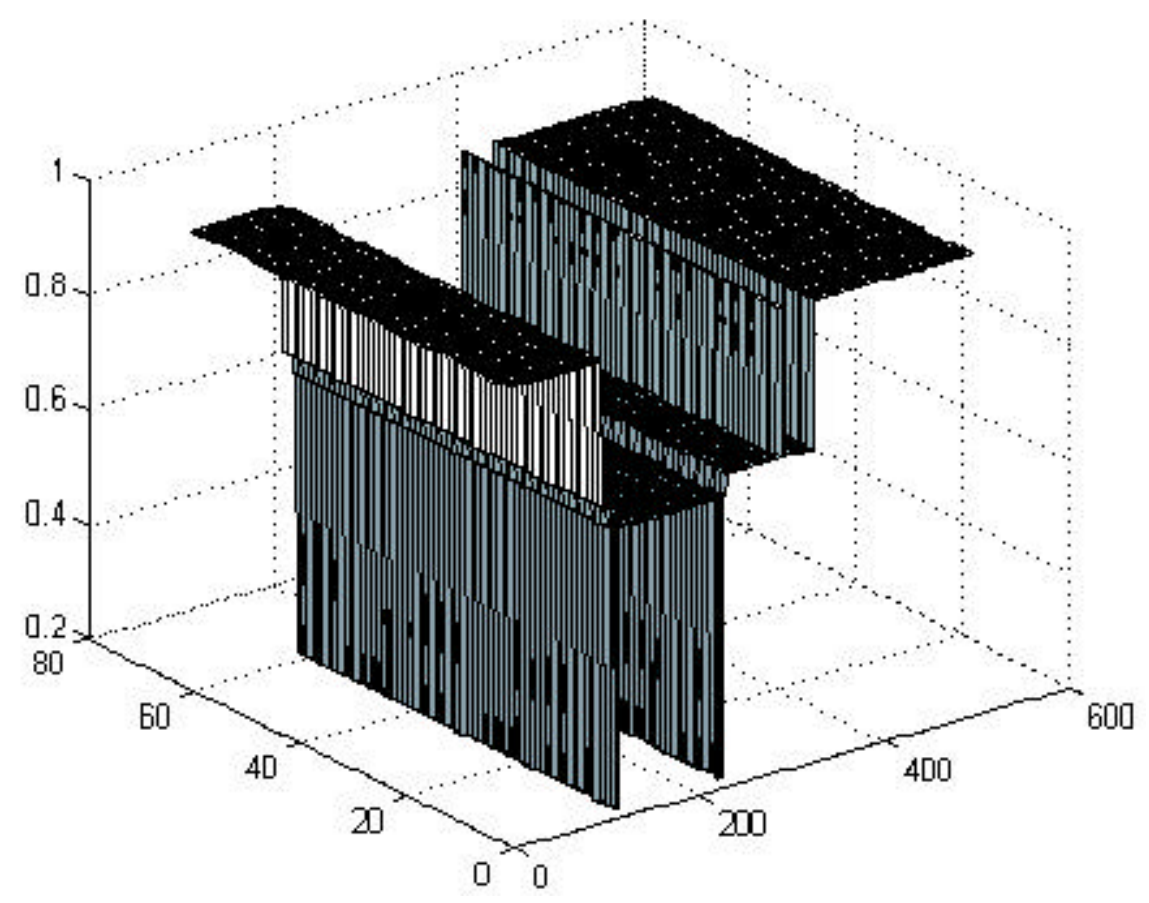

Figure 4.17: Device Transmission Function

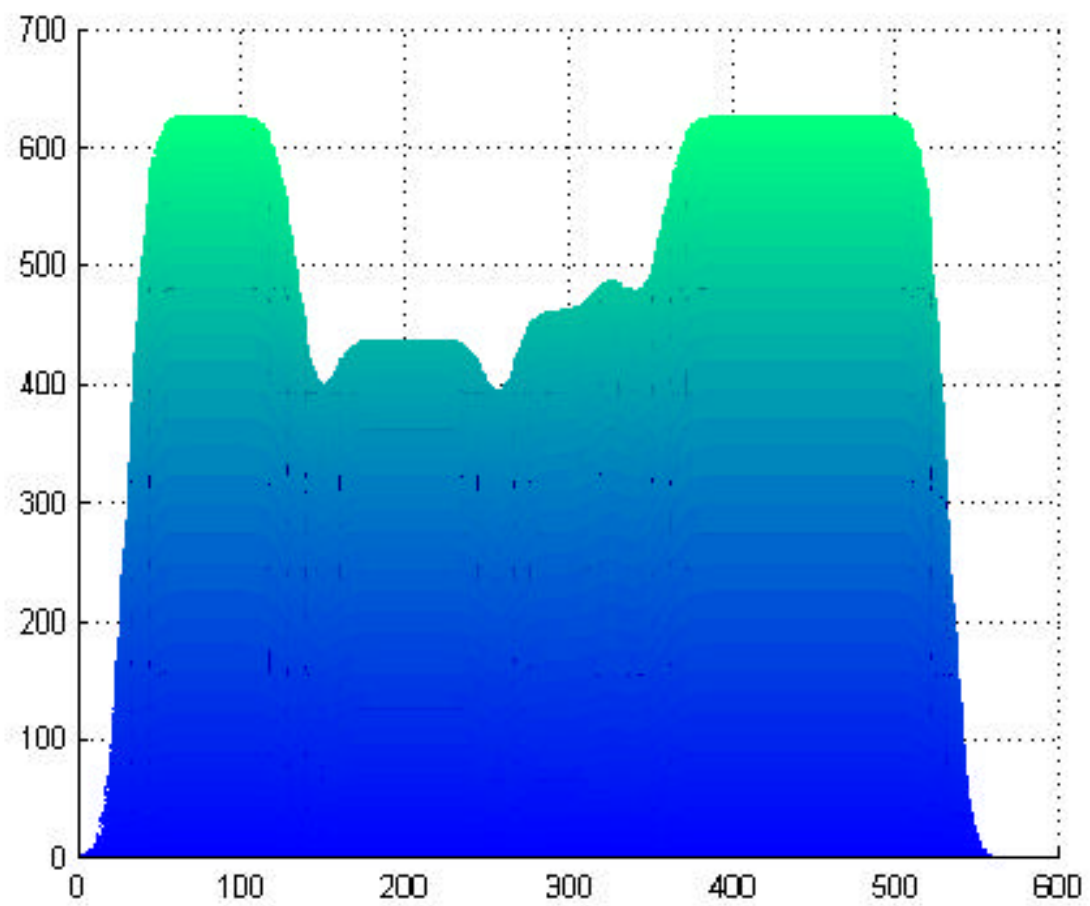

4.18: Convolution (Side View) 
The convolution image shows that the lowest points in the transmission profile occur 125 microns away from each other, under the POLY 2 strips. The goal is to get the sharpest transition in signal when the beam passes from areas with only POLY 0 layers to areas with POLY layers 0,1 , and 2. The GRIN lenses were adjusted to achieve the smallest apparent spot size of the output beam with the device stage in focus on the IR camera display. It should be noted that the binocular microscope used made visual determination of the actual spot size and location difficult, and that the monochromatic nature of the laser diode caused significant diffraction and stray reflections that caused visual location of the spot to be uncertain as well.

To be more certain of the location of the spot, scans were done to find the location of the contact pads. Because the contact pads are metal, transmission should be very low or zero when the spot is under one of them. Alpha step scans were performed to be certain of feature sizes on the MUMPs die. (Figures $4.19 \& 4.20$ )

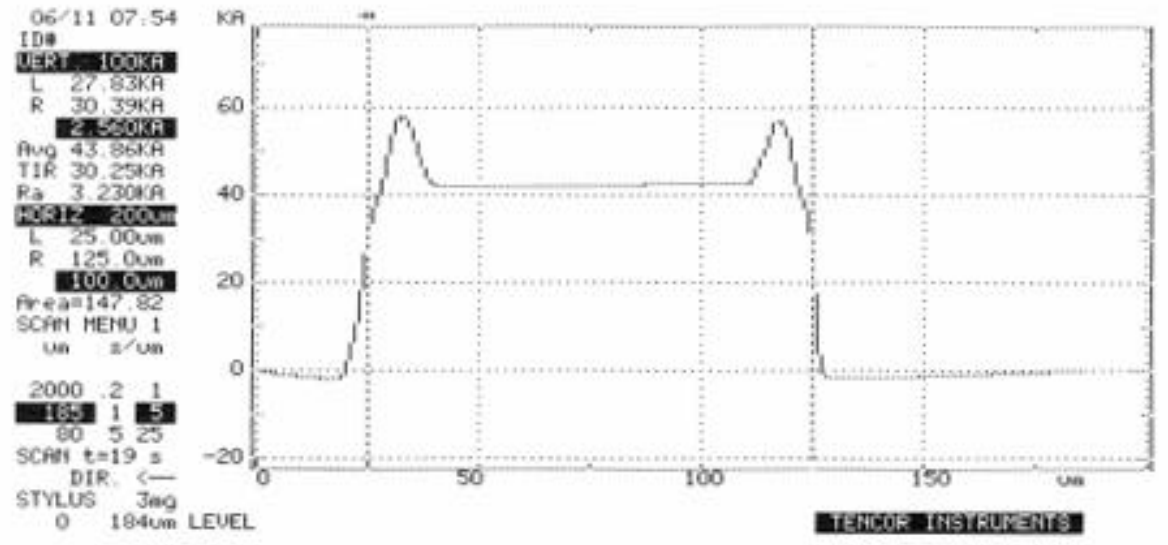

Figure 4.19: Contact Pad Width 


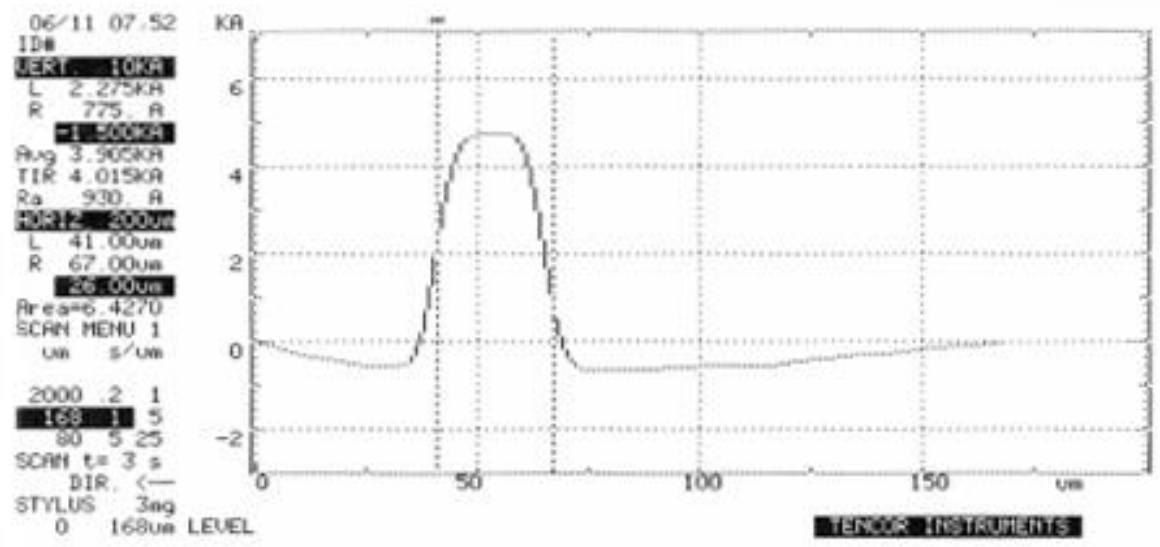

Figure 4.20: Contact Connection Strip Width

If a region of low intensity was discovered, distance comparison with the alpha step scan was done to see if the structure being scanned under is indeed one of the pads. After the scan was completed, the spot was moved back into a position just outside of the pad. The detector fiber was moved outside of the field of view of the microscope and the spot location was observed on the IR camera. Fortunately the microscope optics were such that the "false" spot was in the vicinity of the one scanned, not one of the other three connected to the device. The actual spot was marked on the output screen, and the die was moved so it would be just outside the desired scanning location in the comb split, an unpatterned area of the die. The detector fiber was then moved over the spot focused in the device plane until it read the highest possible output. The detector fiber was lowered as close to the device as possible $(\sim 0.5 \mathrm{~mm})$ to decrease beam divergence. The spot was then scanned under the stage in the same manner as was explained in the image capture tests. Figures 4.21-4.28 show the results of the spot scans under a contact pad for correction, and scans under the device translation stage. These scans were performed with 47 and $8 \mu \mathrm{m}$ core detector fibers and a double GRIN lens setup. A modulated IR source 
(15 $\mathrm{mV}$ modulation, $5 \mathrm{mV}$ offset, $6 \mathrm{~mA}$ input current) was used for to better observe a signal change on the oscilloscope output.

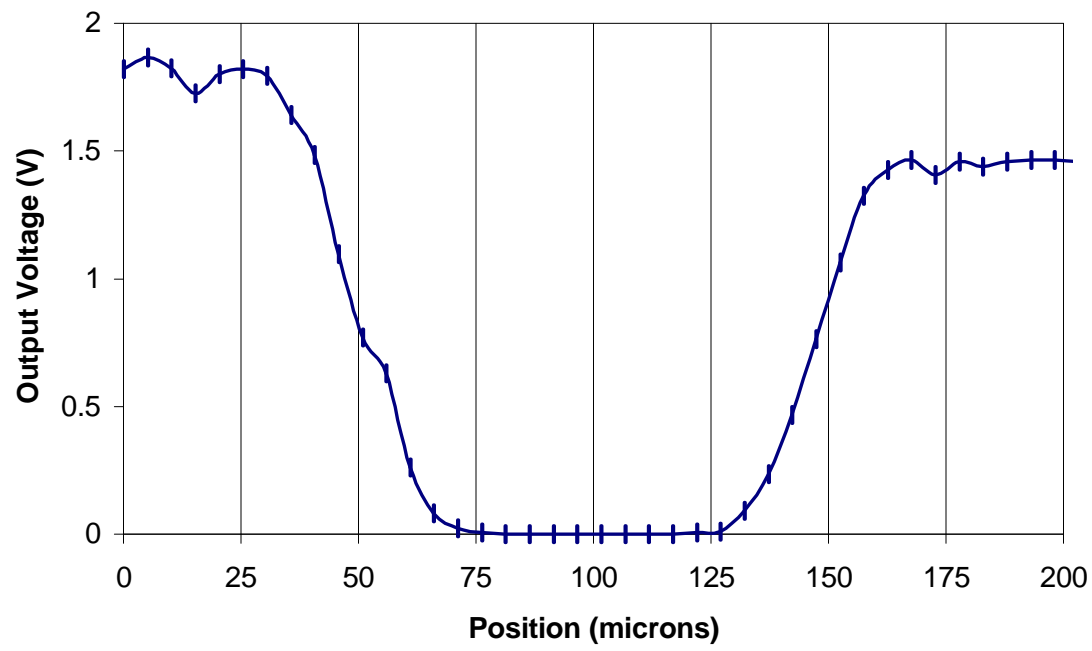

Figure 4.21: Through-Wafer Voltage Under Contact Pad (LD, $47 \mu \mathrm{m}$ detector fiber, double GRIN lenses)

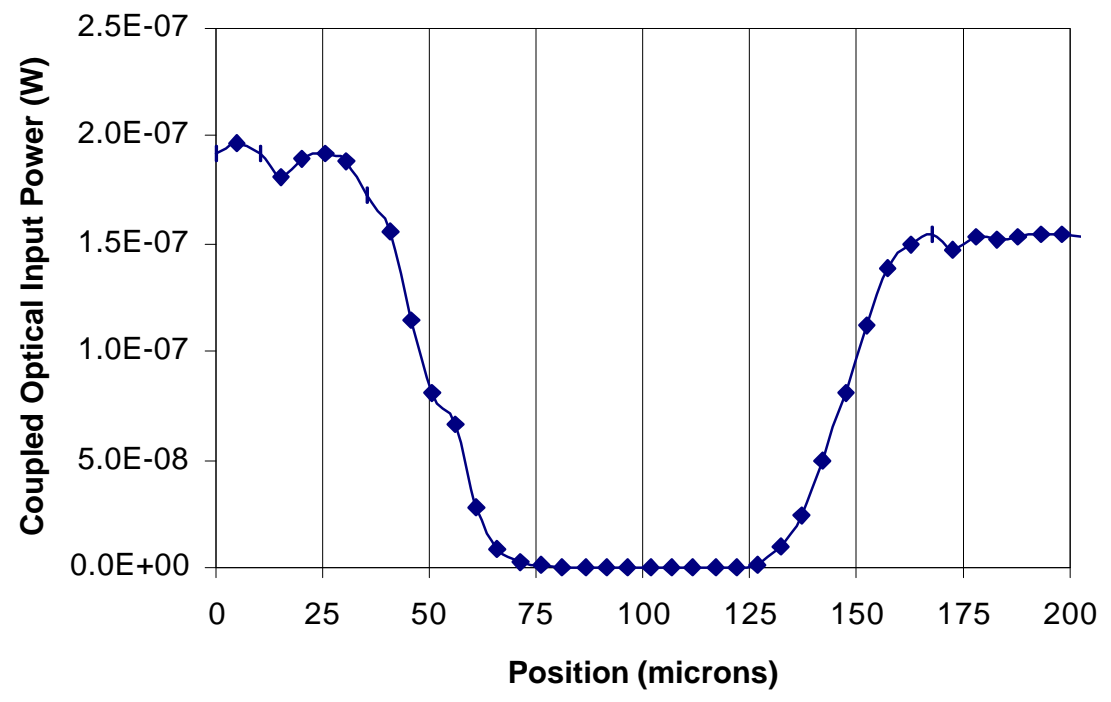

Figure 4.22: Through-Wafer Power Under Contact Pad (LD, $47 \mu \mathrm{m}$ detector fiber, double GRIN lenses) 


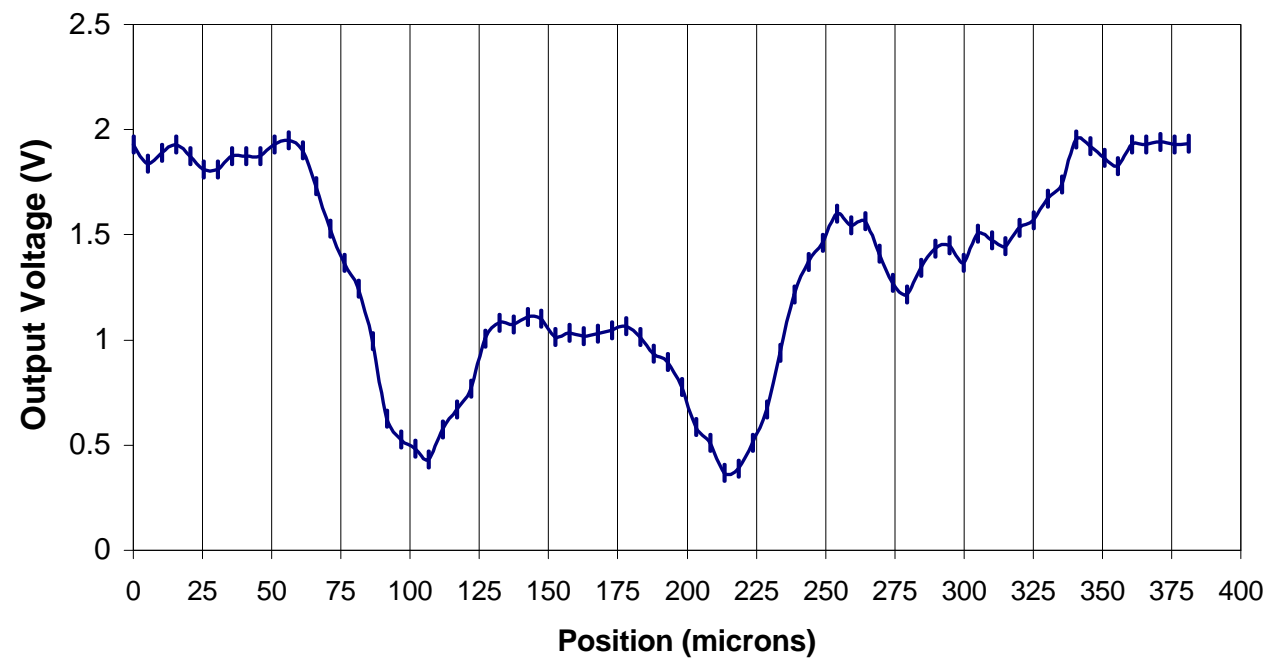

Figure 4.23: Through-Wafer Voltage Under Stage (LD, $47 \mu \mathrm{m}$ detector fiber, double GRIN lenses)

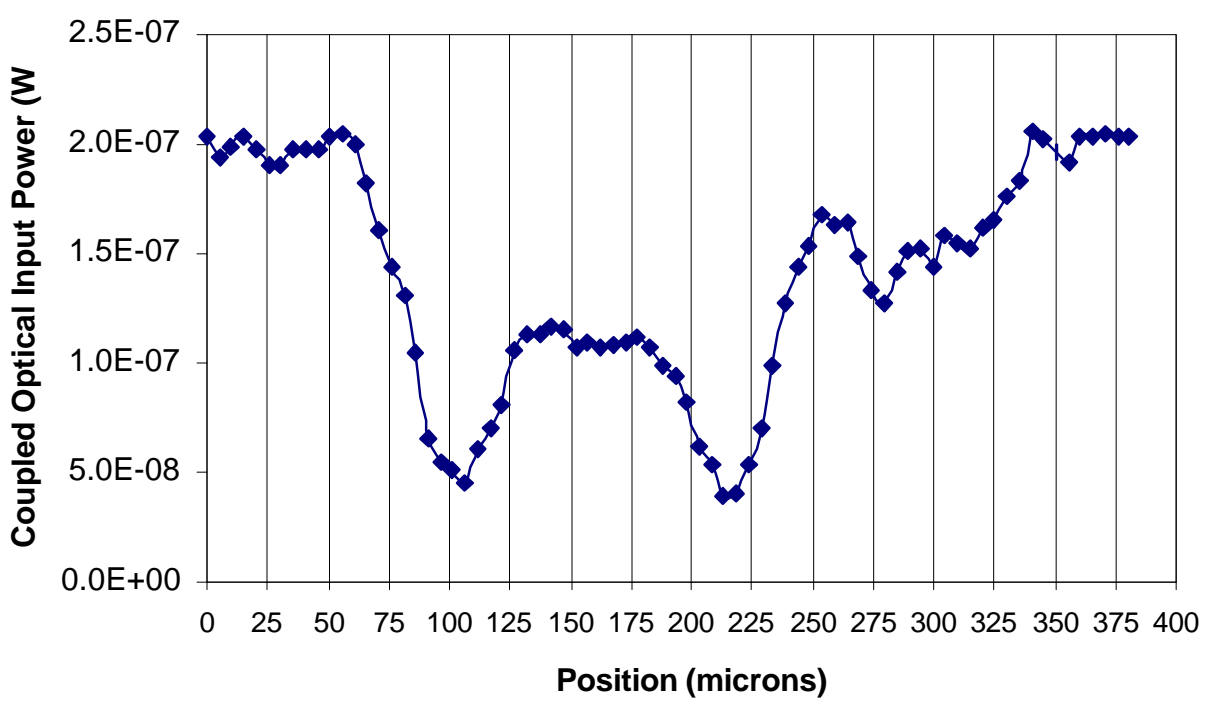

Figure 4.24: Through-Wafer Power Under Stage (LD, $47 \mu \mathrm{m}$ detector fiber, double GRIN lenses) 


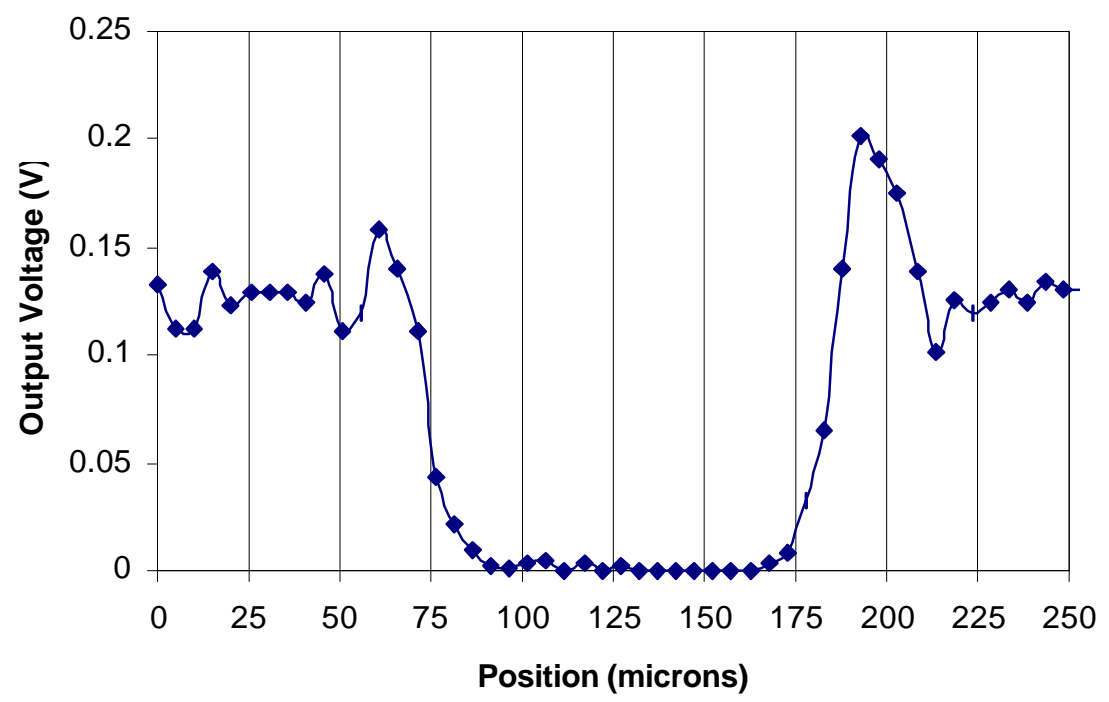

Figure 4.25: Through-Wafer Voltage Under Contact Pad (LD, $8 \mu \mathrm{m}$ detector fiber, double GRIN lenses)

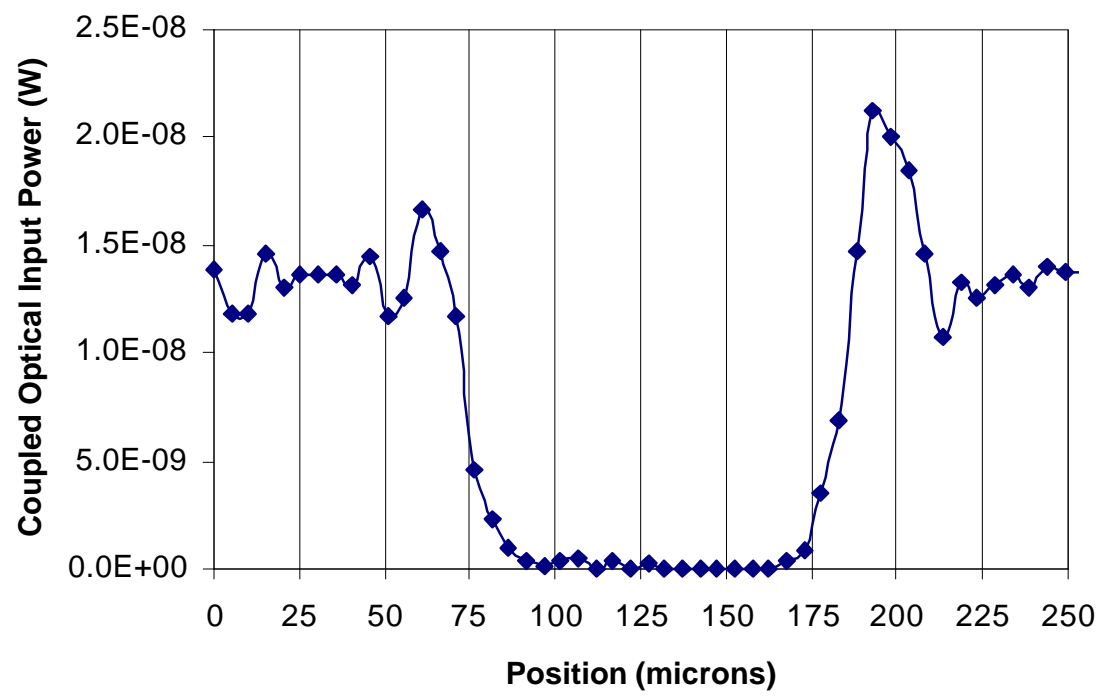

Figure 4.26: Through-Wafer Power Under Contact Pad (LD, $8 \mu \mathrm{m}$ detector fiber, double GRIN lenses) 


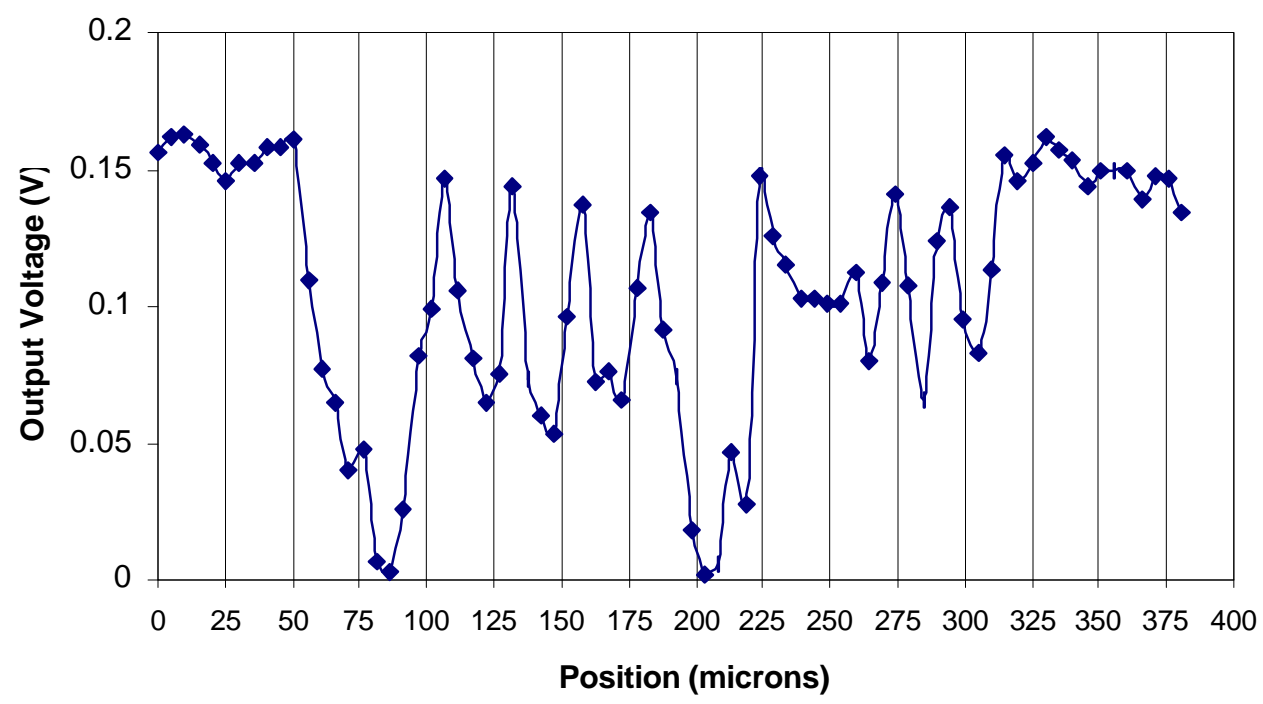

Figure 4.27: Through-Wafer Voltage Under Stage

(LD, $8 \mu \mathrm{m}$ detector fiber, double GRIN lenses)

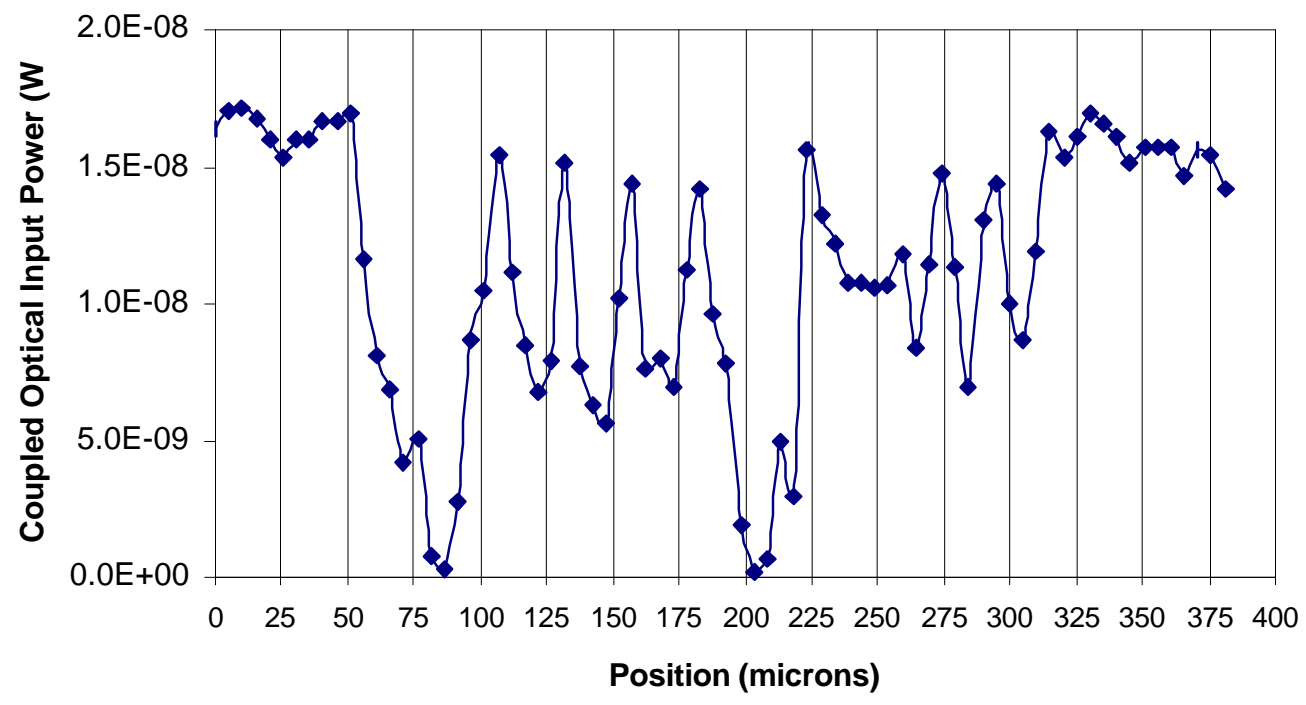

Figure 4.28: Through-Wafer Power Under Stage (LD, $8 \mu \mathrm{m}$ detector fiber, double GRIN lenses) 
These measurements taken coincide with the theoretical convolution results and device feature locations in the area of the scans (Figure 4.29).

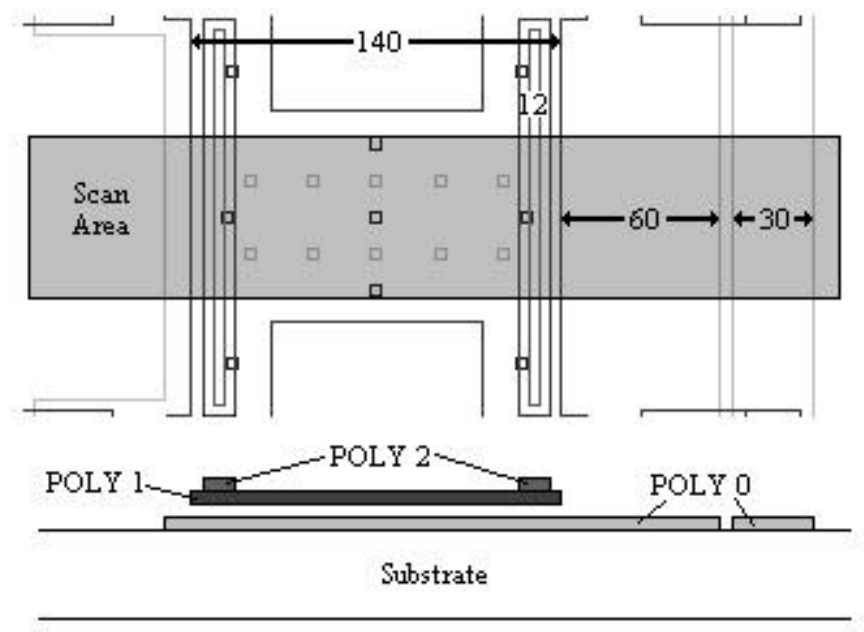

Figure 4.29: Area of IR Scan (All Dimensions in $\mu \mathrm{m}$ )

It can be observed from this series of tests that the laser diode and double GRIN lens setup gave a smaller spot size, and smaller core detector fibers gave signal with significantly more spatial information. These conclusions are drawn because of the increase in sharpness in transition from a signal read through unpatterned silicon to a signal read under the device stage. It is clear that the contact pads are $100 \mu \mathrm{m}$ wide and that the distance between the POLY 2 strips on the device stage is $140 \mu \mathrm{m}$. Sharp spikes in intensity that occur before and after features are most likely diffraction effects caused by the coherent laser diode light source at the edge of the features. Spikes occurring between 80 and $200 \mu \mathrm{m}$ in Figure 4.28 may be a result of light passing through the etch holes in the POLY 1 layer of the stage. During the $8 \mu \mathrm{m}$ detector scan, there was a $\mathrm{x} 5$ change in intensity around the $225 \mu \mathrm{m}$ location in Figure 4.28. This is the location of the 
POLY 2 strip. The sharp decrease in signal over a short distance also implies very small spot size, $\sim 10 \mu \mathrm{m}$ in diameter.

Transmission percentages agreed with theoretical results discussed in section 4.1 as well. The following table relates the percentage of transmitted light from unpatterned silicon to transmission under various layers of polysilicon for both the 8 and the $47 \mu \mathrm{m}$ scans. The layer locations are based on comparisons between Figures $4.23 \& 4.24$ and $4.27 \& 4.28$ and locations of features in the scan area shown in Figure 4.29.

Table 4.1: Transmission Percentages

\begin{tabular}{|c|c|c|c|}
\hline & POLY 0 & POLY 0/air/POLY 1 & $\begin{array}{c}\text { POLY 0/air/POLY } \\
\text { 1/POLY 2 }\end{array}$ \\
\hline $\begin{array}{c}\text { Theoretical } \\
\text { Values }\end{array}$ & $74 \%$ & $70 \%$ & $21 \%$ \\
\hline $\begin{array}{c}47 \text { micron } \\
\text { detector }\end{array}$ & $60-75 \%$ & $50-65 \%$ & $20 \%$ \\
\hline $\begin{array}{c}8 \text { micron } \\
\text { detector }\end{array}$ & $66 \%$ & $31-53 \%$ & $2 \%$ \\
\hline
\end{tabular}

Transmission data measured with the $47 \mu \mathrm{m}$ detector fiber matches the theoretical values more closely. The $8 \mu \mathrm{m}$ detector fiber proved to be unstable in the experimental setup, causing unwanted noise. The low values measured by the $8 \mu \mathrm{m}$ detector are most likely a result of diffraction effects and experimental system instability. If the system were better stabilized, diffraction effects from the $8 \mu \mathrm{m}$ fiber could be used to obtain a stronger signal.

Initially, three different detector fiber core diameters, 100,47 , and $8 \mu \mathrm{m}$, were used to scan under the device stage. However, due to uncertainties in spot location theses 
scans passed under an area different than that shown in Figure 4.29. Figures 4.30-4.37 show the results of these tests. The LED and laser diode were modulated as described previously.

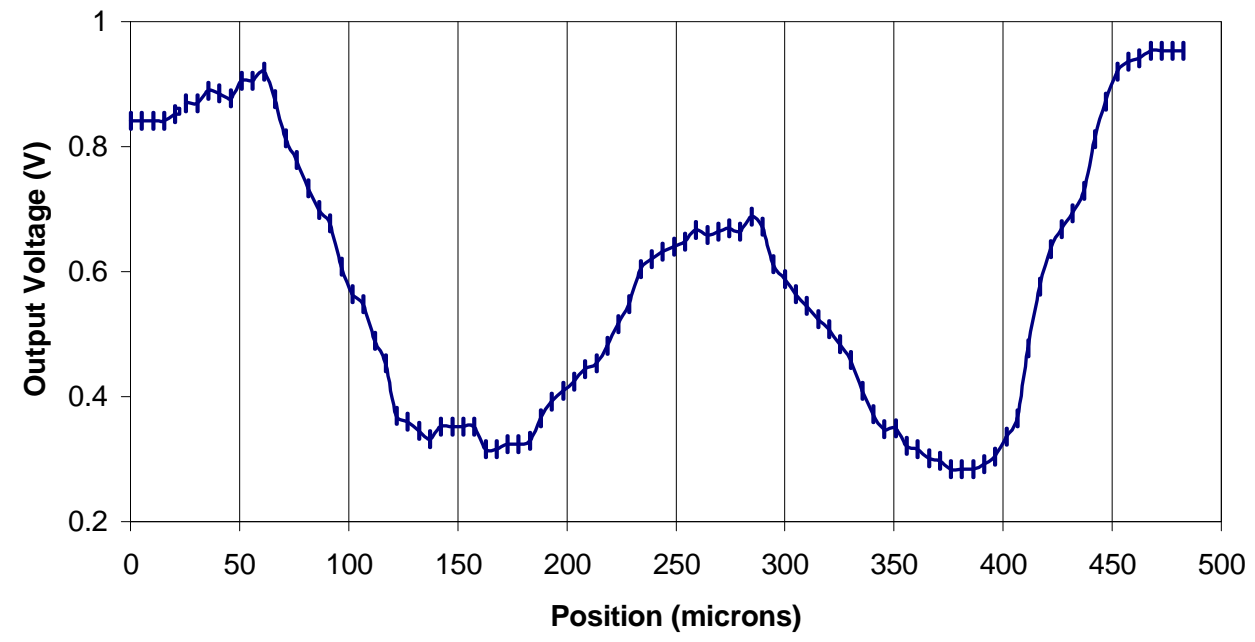

Figure 4.30: Through- Wafer Voltage (LED, $100 \mu \mathrm{m}$ detector fiber, single GRIN lens)

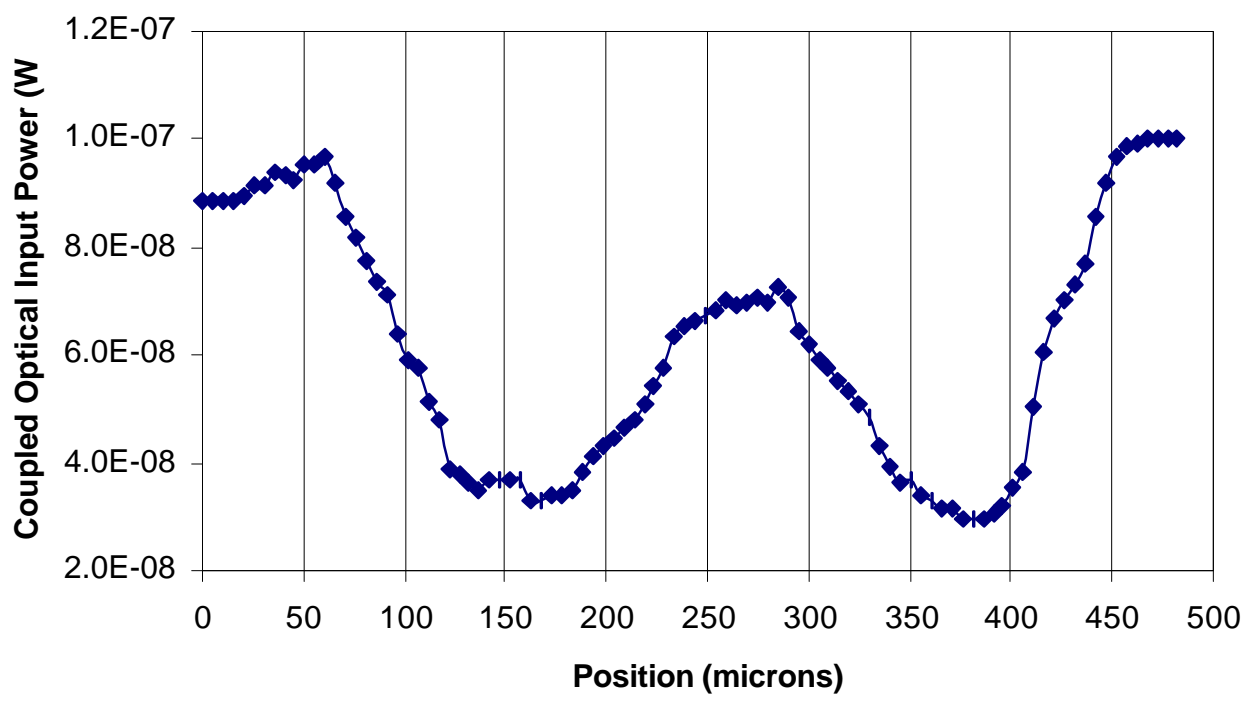

Figure 4.31:Through- Wafer Power (LED, $100 \mu \mathrm{m}$ detector fiber, single GRIN lens) 


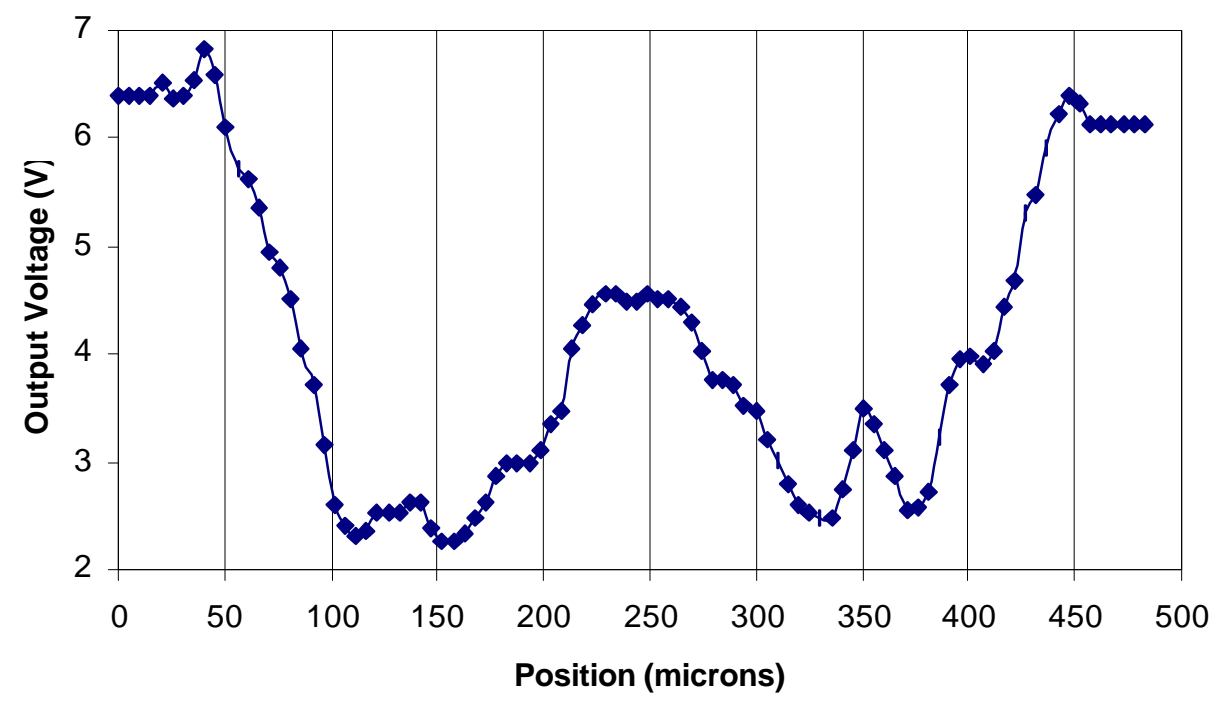

Figure 4.32: Through-Wafer Voltage

(LD, $100 \mu \mathrm{m}$ detector fiber, double GRIN lenses)

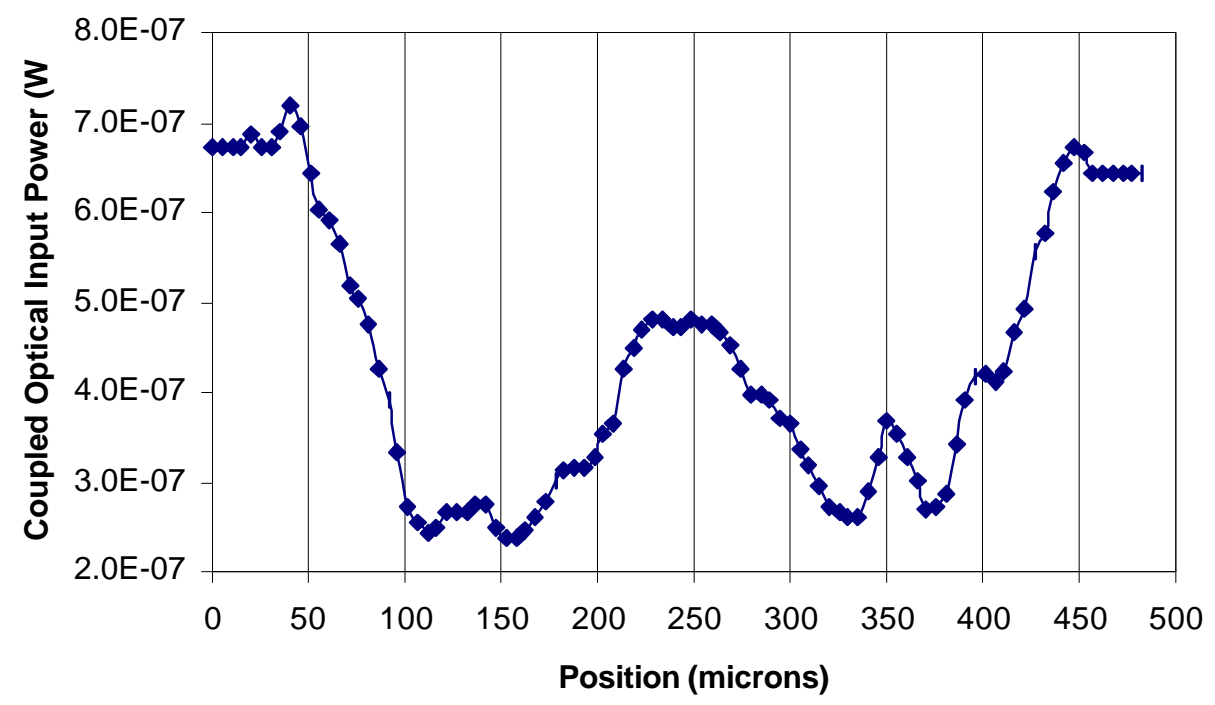

Figure 4.33: Through-Wafer Power

(LD, $100 \mu \mathrm{m}$ detector fiber, double GRIN lenses) 


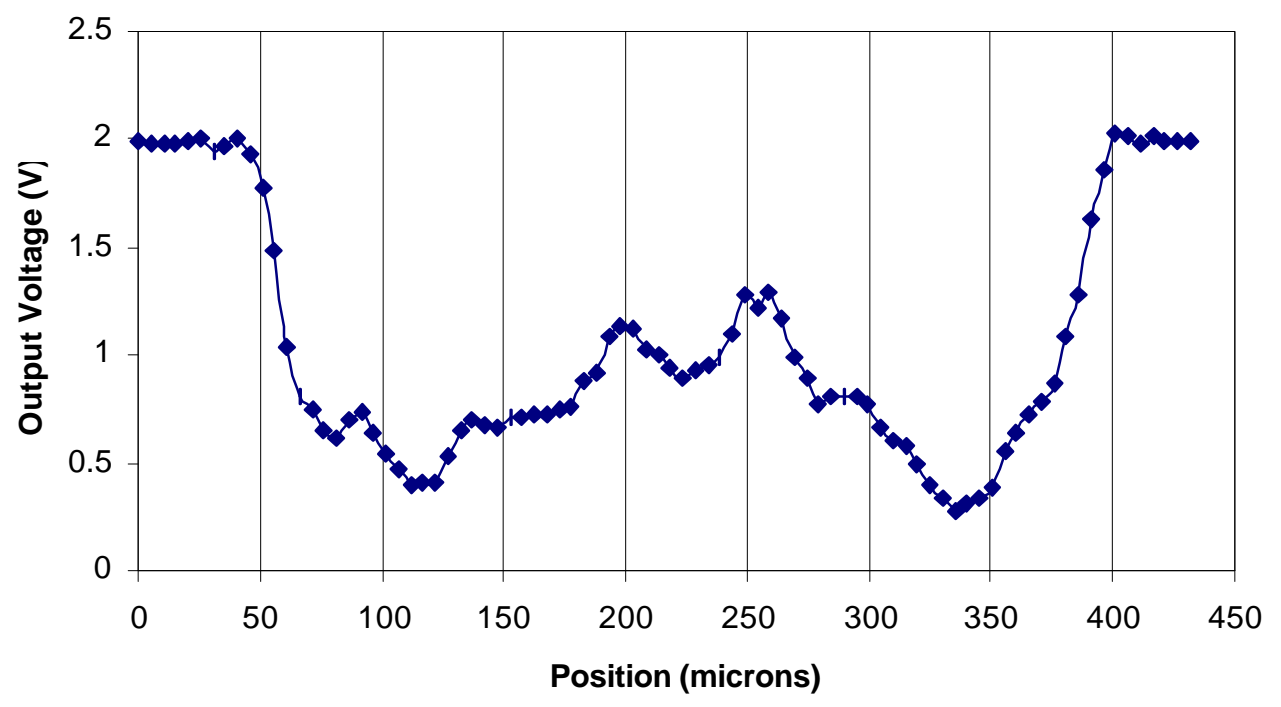

Figure 4.34: Through-Wafer Voltage

(LD, $47 \mu \mathrm{m}$ core detector, double GRIN lenses)

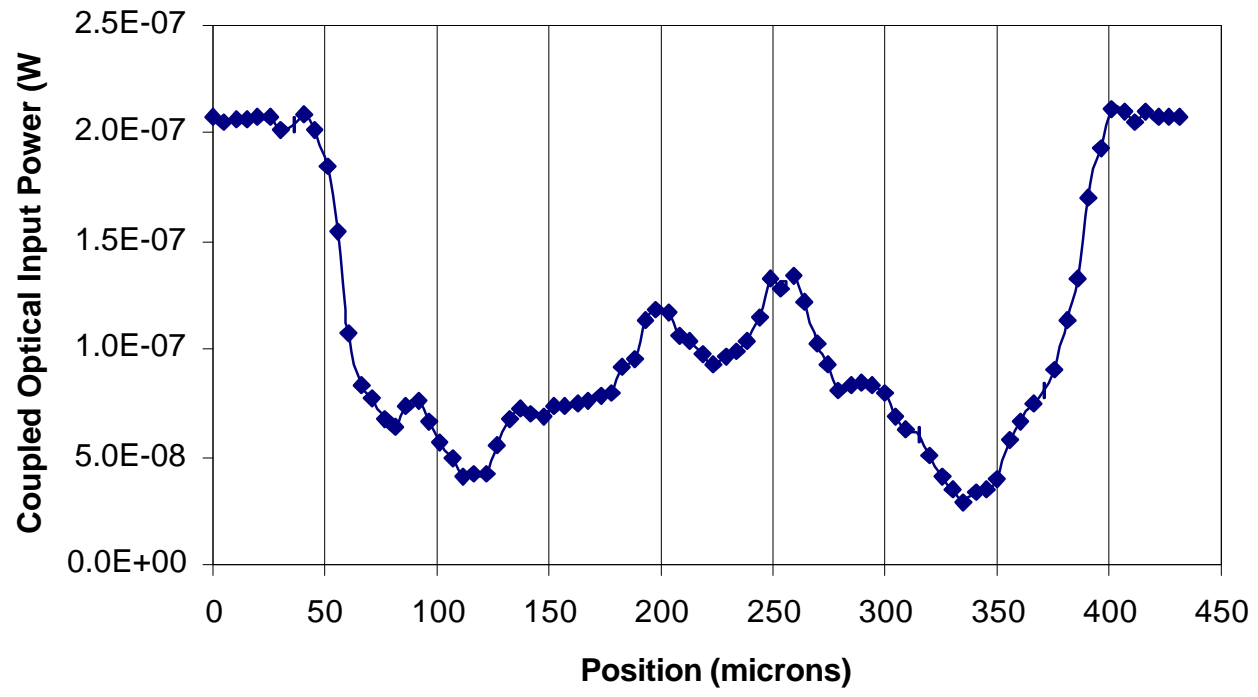

Figure 4.35: Through-Wafer Power

(LD, $47 \mu \mathrm{m}$ core detector, double GRIN lenses) 


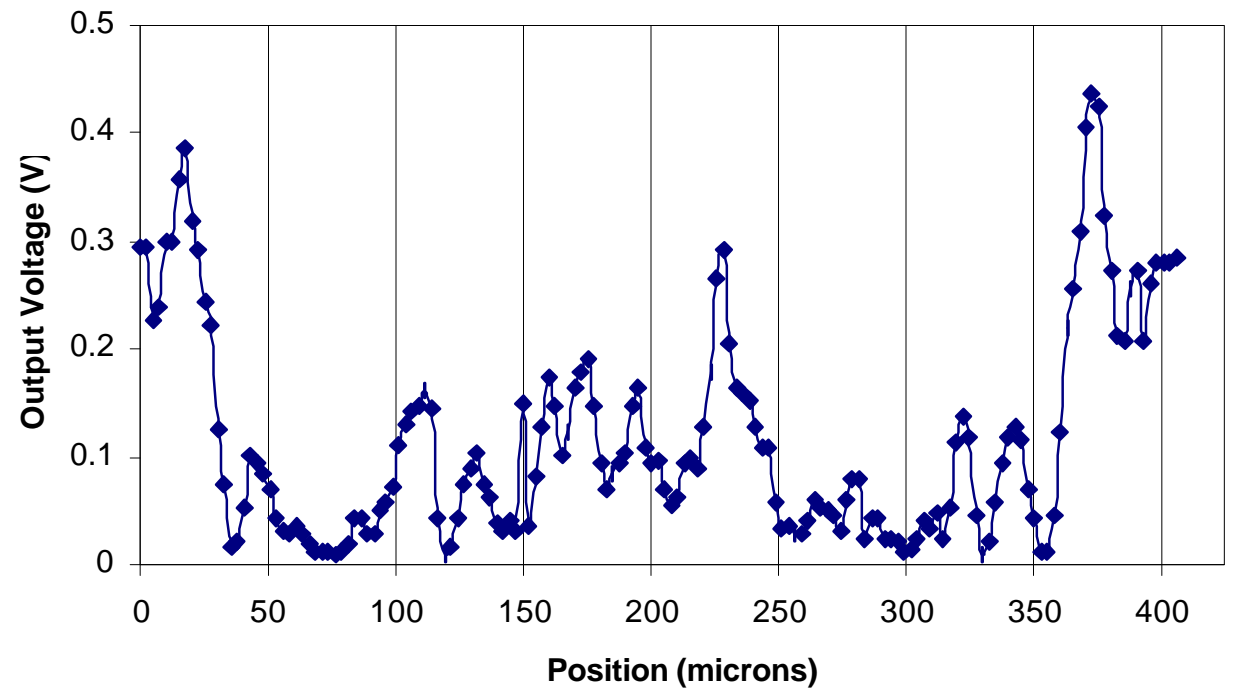

Figure 4.36: Through-Wafer Voltage

(LD, $8 \mu \mathrm{m}$ detector fiber, double GRIN lenses)

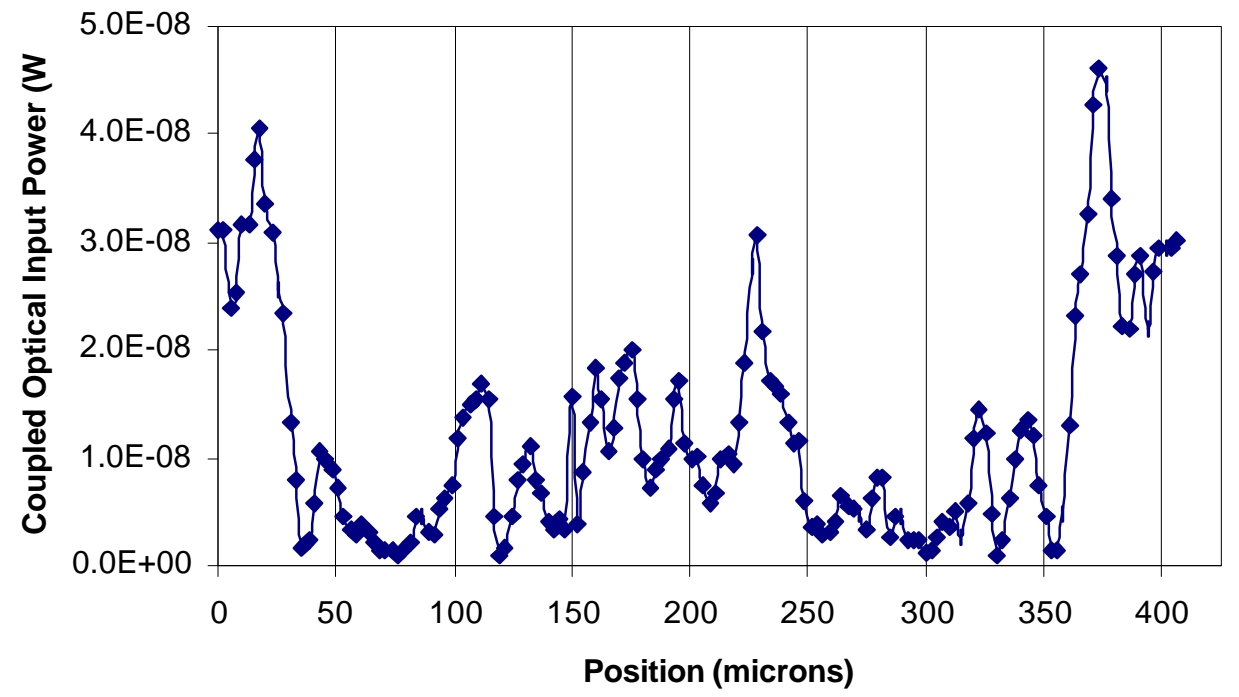

Figure 4.37: Through-Wafer Power (LD, $8 \mu \mathrm{m}$ detector fiber, double GRIN lenses) 
Upon further inspection, one can see that the measured outputs agree somewhat with the theoretical convolution results in shape, but not in the distances. Figure 4.29 lends further proof that the wrong area had been scanned. Both this figure and the convolution results show that the lowest transmission intensities should occur approximately $130 \mu \mathrm{m}$ apart. The power and voltage measurement results illustrated in Figures 4.30-4.37 show these minimums to be as high as $375 \mu \mathrm{m}$ apart, more than twice the distance expected. Since the dimensions indicated in the LASI layout were verified by the alpha step scans (Figures $4.19 \& 4.20$ ), the area that the spot scanned when the tests were performed must not have been the area described in Figure 4.29. Figure 4.38 shows the suspected area of these incorrect scans.

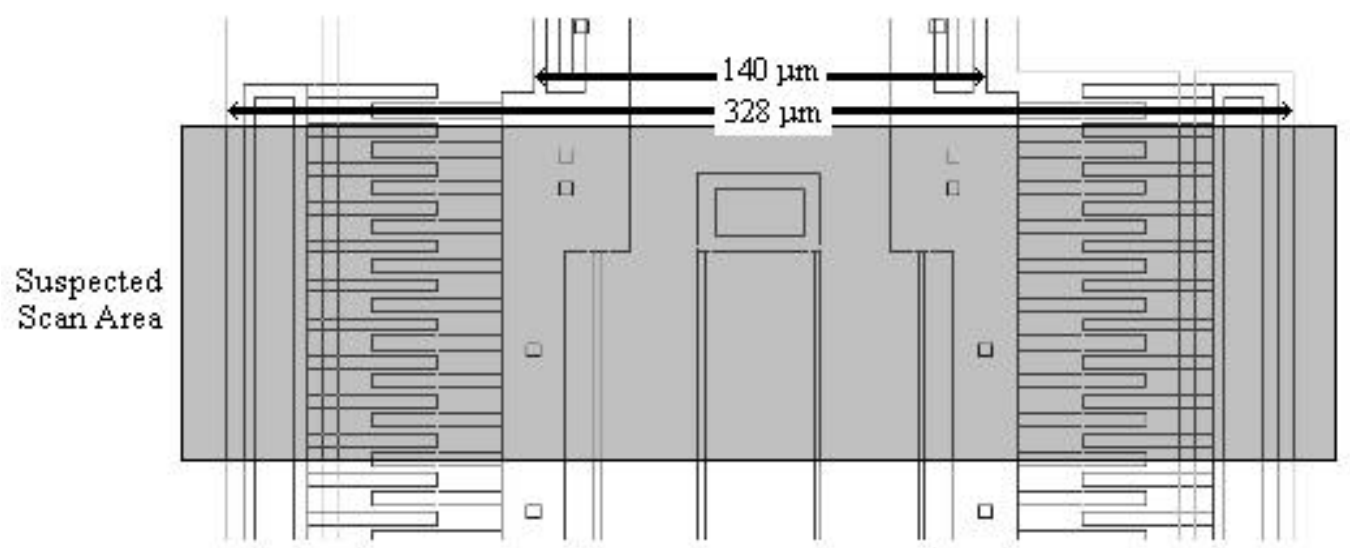

Figure 4.38: Area of Incorrect Scans

This is another example in which the binocular microscope is very unreliable for visual determination of IR spot location, and shows that the spot may be focused in the device plane somewhere other than the location viewed with the camera.

These scans do show the improvement in signal as the size of the detector fiber is decreased. More details of the device features are visible in the scans done with the 47 and $8 \mu \mathrm{m}$ detectors. They also illustrate the smaller spot sizes achievable with the laser 
diode and the double grin lens setup. This is evident because of the sharp decrease in intensity at the feature edges, but is enhanced by diffraction effects.

\subsection{Optical Monitoring of Static Deflection}

To observe the effects that interruption of the optical probe by the moving device stage had on the through-wafer signal, the stage was actuated by a DC voltage. To accomplish this task, the MEMS device had to be probed and monitored by the detector simultaneously. This added another degree of complexity to the already densely packed experimental setup. Luckily, the small size of the detector fiber made it suitable for maneuvering in between the probe tips. Since the $8 \mu \mathrm{m}$ detector fiber gave the sharpest signal, it was the only fiber probe used in these tests. The laser diode was driven at 10 mA DC. As with the stationary stage and static displacement measurements, before testing the spot was scanned under a contact pad and its position was verified. Once the device was in the correct location, the probes were lowered and the detector fiber was moved into position. Because one side of the device slit area had POLY 0, POLY1, and POLY2 layers in close proximity, the other side of the device was used in the frequency validation tests. This side had a large area of POLY 0, further lowering the intensity of the spot, but created a less cluttered signal due to the absence of the POLY 0-nitride step located on the other side of the stage. The next step was to apply power to the device. While applying $\pm 17 \mathrm{~V}$ to the stator combs, the stage voltage was varied from positive to negative $15 \mathrm{~V}$ and the output data was recorded. Starting spot location was determined by adjusting the coupled output and detector until a slight decrease in intensity was found. 
Position verification was performed by the same method discussed earlier. Figure 4.39 shows the results of this measurement, and Figure 4.40 shows the $0 \mathrm{~V}$ spot location.

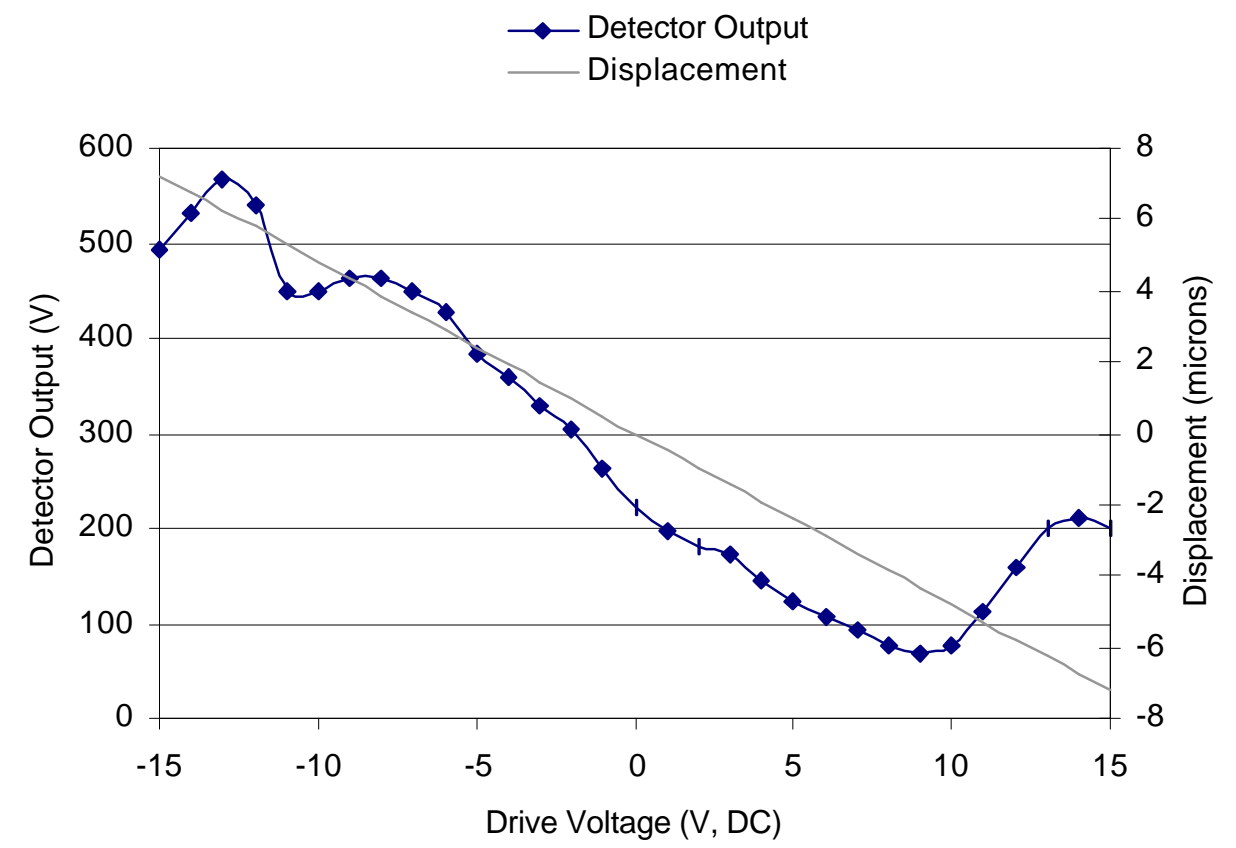

Figure 4.39: Static Displacement Output Analysis

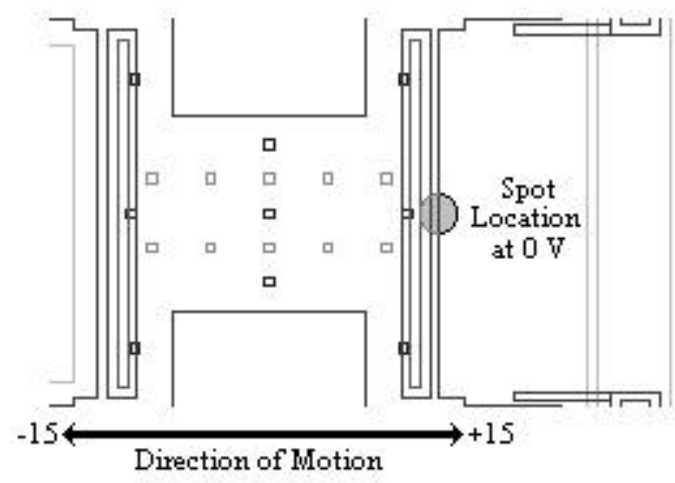

Figure 4.40: Spot location at $0 \mathrm{~V}$ 
It is clear from the output data that as the stage moved in the $-15 \mathrm{~V}$ direction, intensity increased, and decreased in the $+15 \mathrm{~V}$ direction. The theoretical displacement data confirms the direction of motion and, to some degree, the actual distances moved. As the stage moved in the $+15 \mathrm{~V}$ direction, intensity decreased, reached a minimum, and increased again. This is most likely a result of the monolithic structure passing completely over the spot and allowing light to pass through to the other side. This structure is $12 \mu \mathrm{m}$ wide, but the stage theoretically travels only $8 \mu \mathrm{m}$ in both directions, for a total swing of $16 \mu \mathrm{m}$. This could be explained by diffraction effects, spot and stage feature geometries, or uncertainties in spot location. It also may be because the device stage may perform better than was predicted in the theoretical analysis.

\subsection{Optical Monitoring During MEMS Actuation}

The time dependent position of an electrostatically driven MEMS device was monitored next. This means that the stage of the device moved continuously while being monitored by the optical probe. Input voltage levels and frequencies were kept low because the devices often experienced catastrophic failure when the stage power supply was turned on at high voltages and frequencies. After the device was determined to be working properly, the detector was moved into position over the spot located in the POLY 1 region. Once the highest intensity of the spot was read by the detector, the fiber and spot were moved just underneath the edge of the device stage. The edge of the device stage was determined by the output signal on the oscilloscope being the same as that of the modulated stage signal. Spot position, frequency, modulation voltage, and stator 
voltages were varied, and data was recorded to observe how the output signal would vary as device inputs were changed.

\subsubsection{Spot Position Variation}

The first test performed was the observation of how the output waveform varied as spot location was changed. After the spot was situated in the starting position described earlier, it was moved under the POLY 2 monolith and out the other side. Three different oscilloscope plots were captured, illustrated in Figures 4.41-4.43. Input device voltages were $\pm 15 \mathrm{~V}$ on the stator combs and a $5 \mathrm{~V}, 1 \mathrm{kHz}$ on the stage.

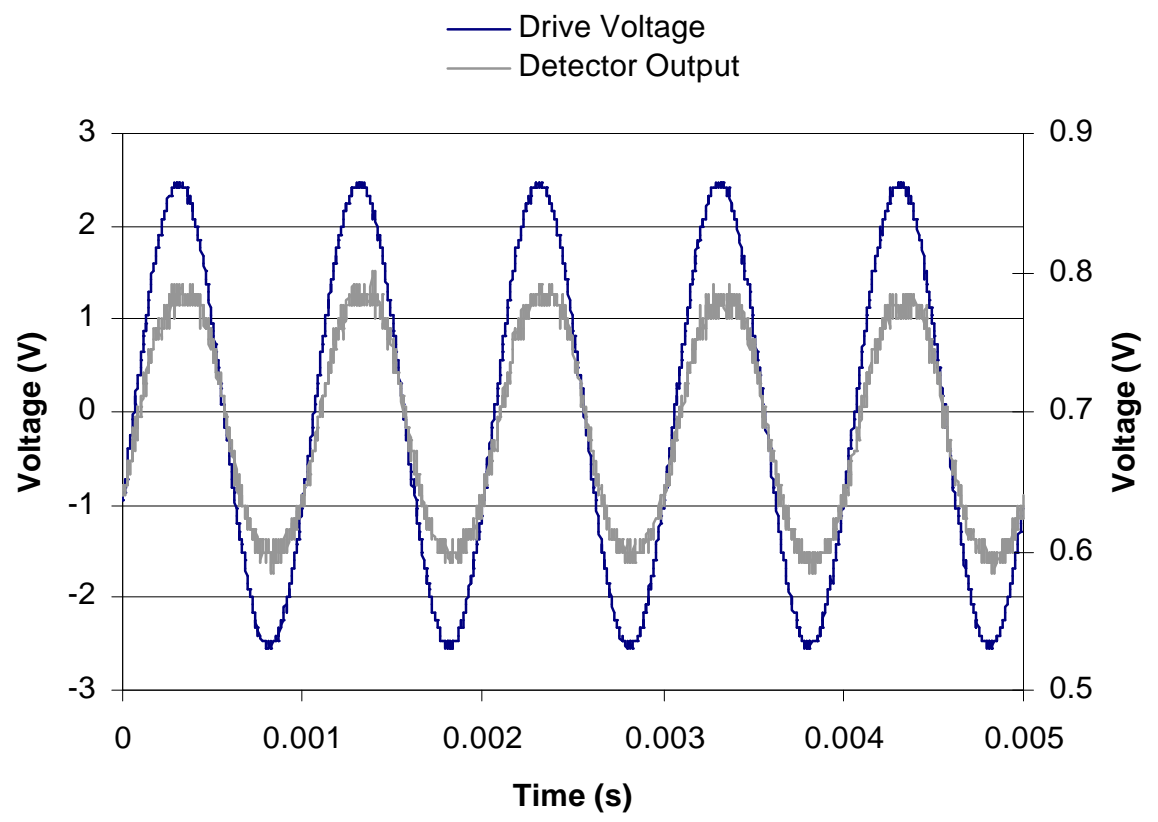

Figure 4.41: Waveform with Spot on Outside Edge of Monolith 


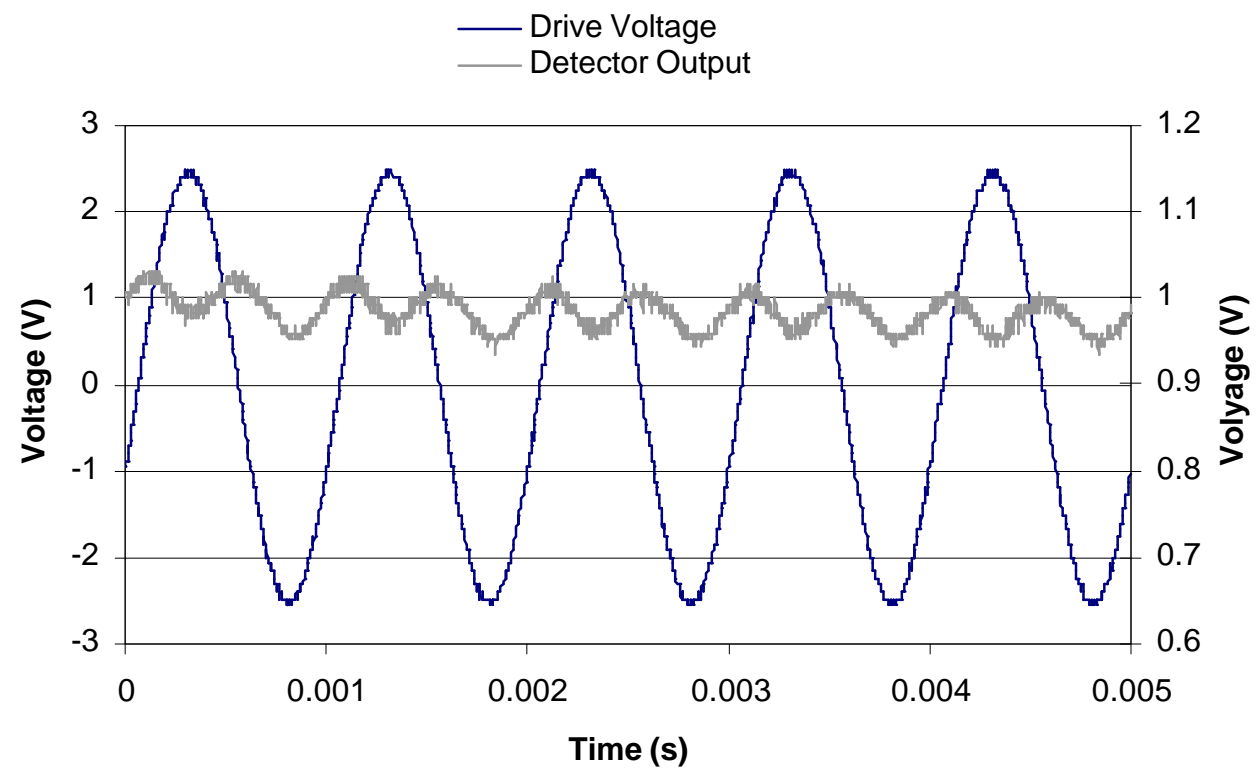

Figure 4.42: Waveform with Spot in Middle of Monolith

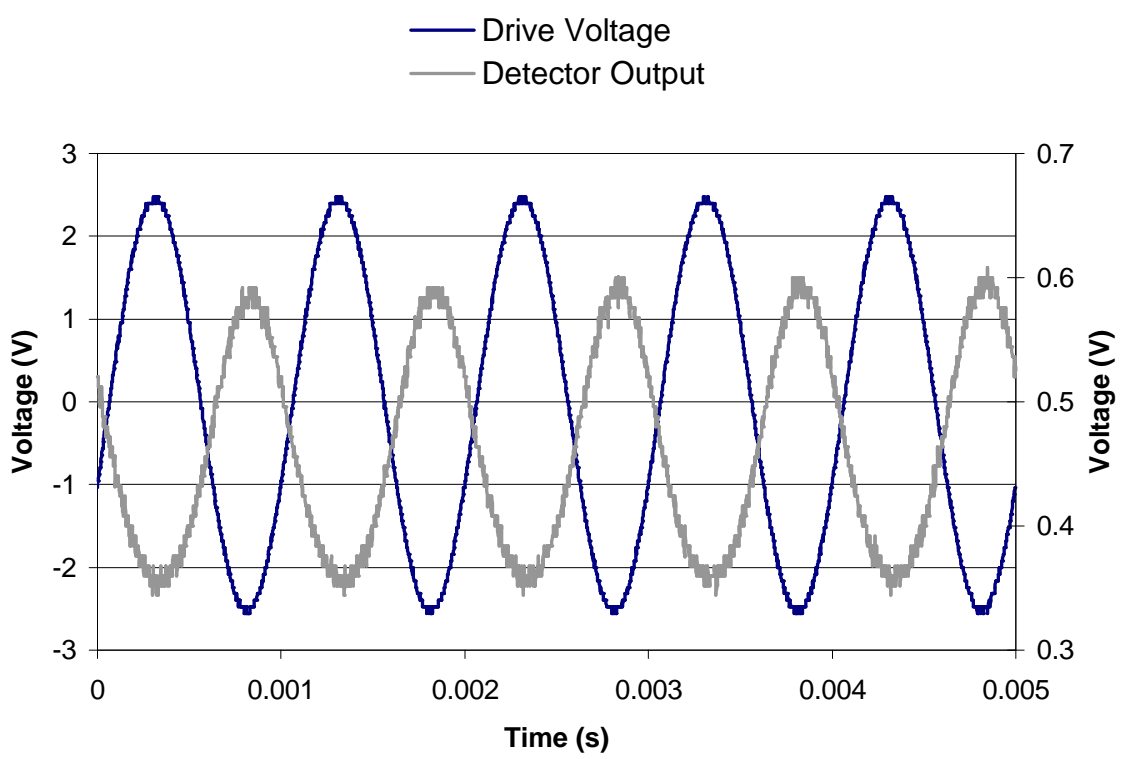

Figure 4.43: Waveform with Spot on Inside Edge of Monolith 
As the spot moves underneath the monolithic structure, the output waveform doubles in frequency and experiences a phase shift on the other side. This corresponds with the direction of motion of the stage. The DC level (at output waveform peak) is higher on the outside than on the inside because of the POLY1 layer present on the inside of the POLY 2 strip. It is highest when covered by the monolith. This is most likely a result of diffraction effects discussed earlier.

\subsubsection{Frequency Variation}

To test the through-wafer setup frequency measuring capability, the input frequency was varied from $500 \mathrm{~Hz}$ to $5 \mathrm{kHz}$ and the output frequency was observed. In these tests the laser diode was driven with a $10 \mathrm{~mA}$ DC signal, and the device was driven at $\pm 10 \mathrm{~V}, 10 \mathrm{~V}_{\mathrm{p}-\mathrm{p}}$. Figures 4.44-4.47 show how the output waveform varied over certain values of the frequency range and Figure 4.48 plots modulation depth as a function of input frequency. The values in the figure represent a normalized difference between maximum output without interruption and output with stage interference, which is the extent to which the signal is decreased by the presence of the stage in the path of the optical probe. Modulation depth was determined by dividing maximum peak output (DC level) by minimum peak output. 


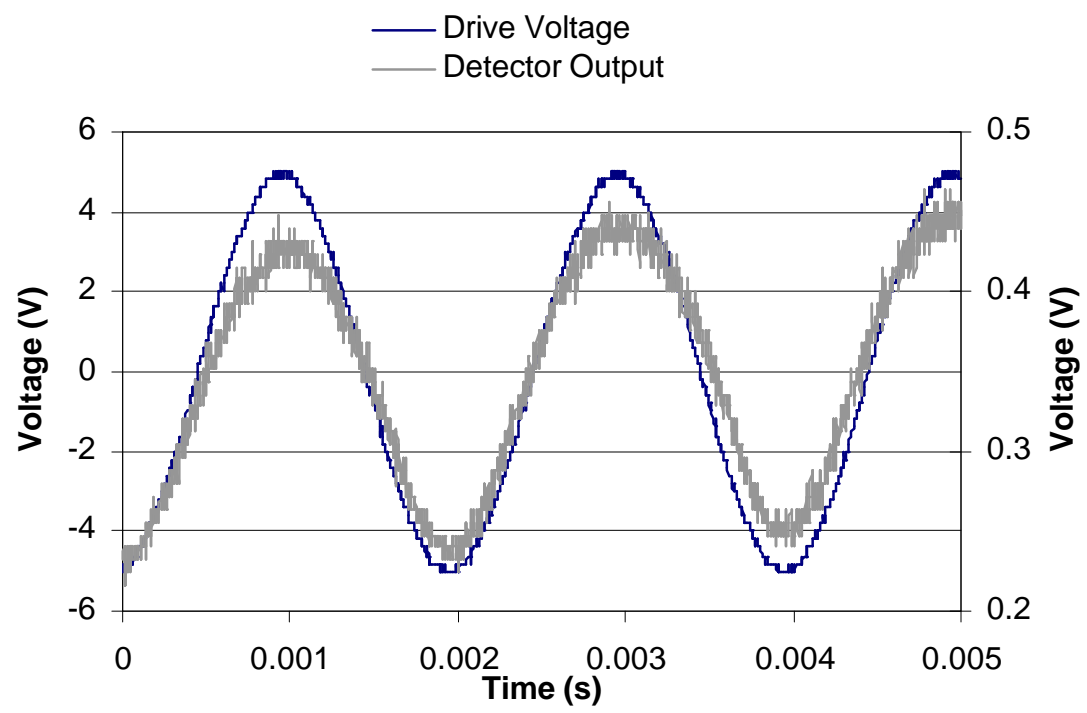

Figure 4.44: Output Comparison at $500 \mathrm{~Hz}$

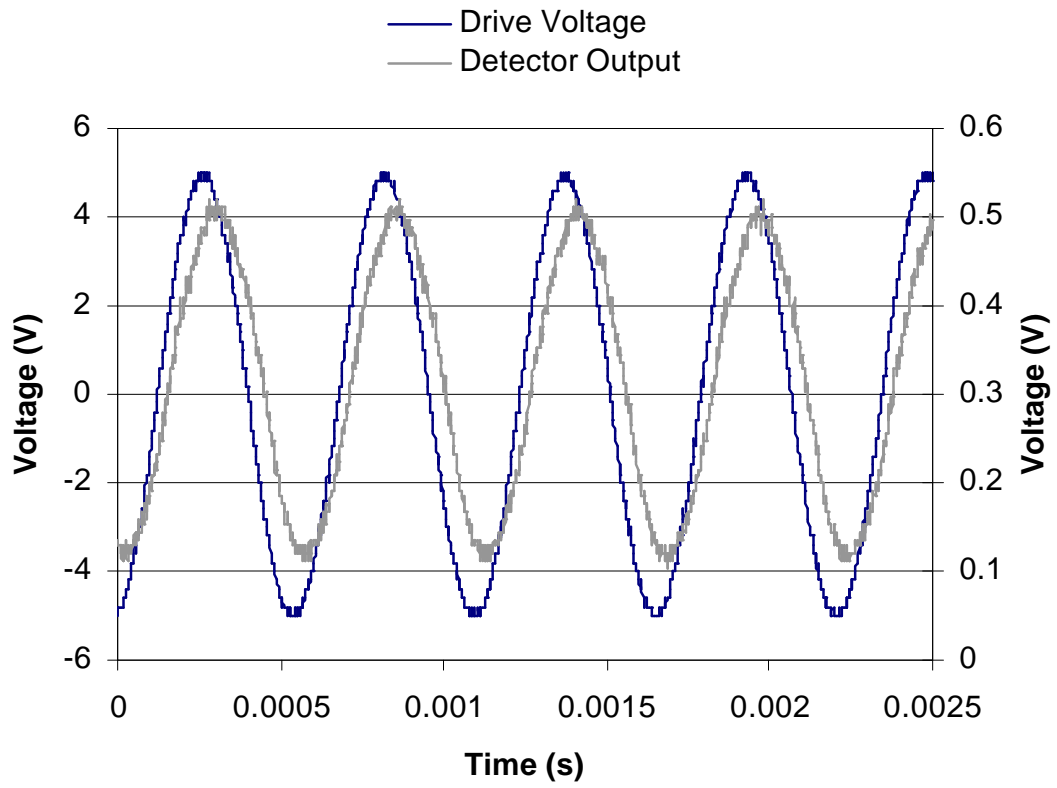

Figure 4.45: Output Comparison at $1.8 \mathrm{kHz}$ 


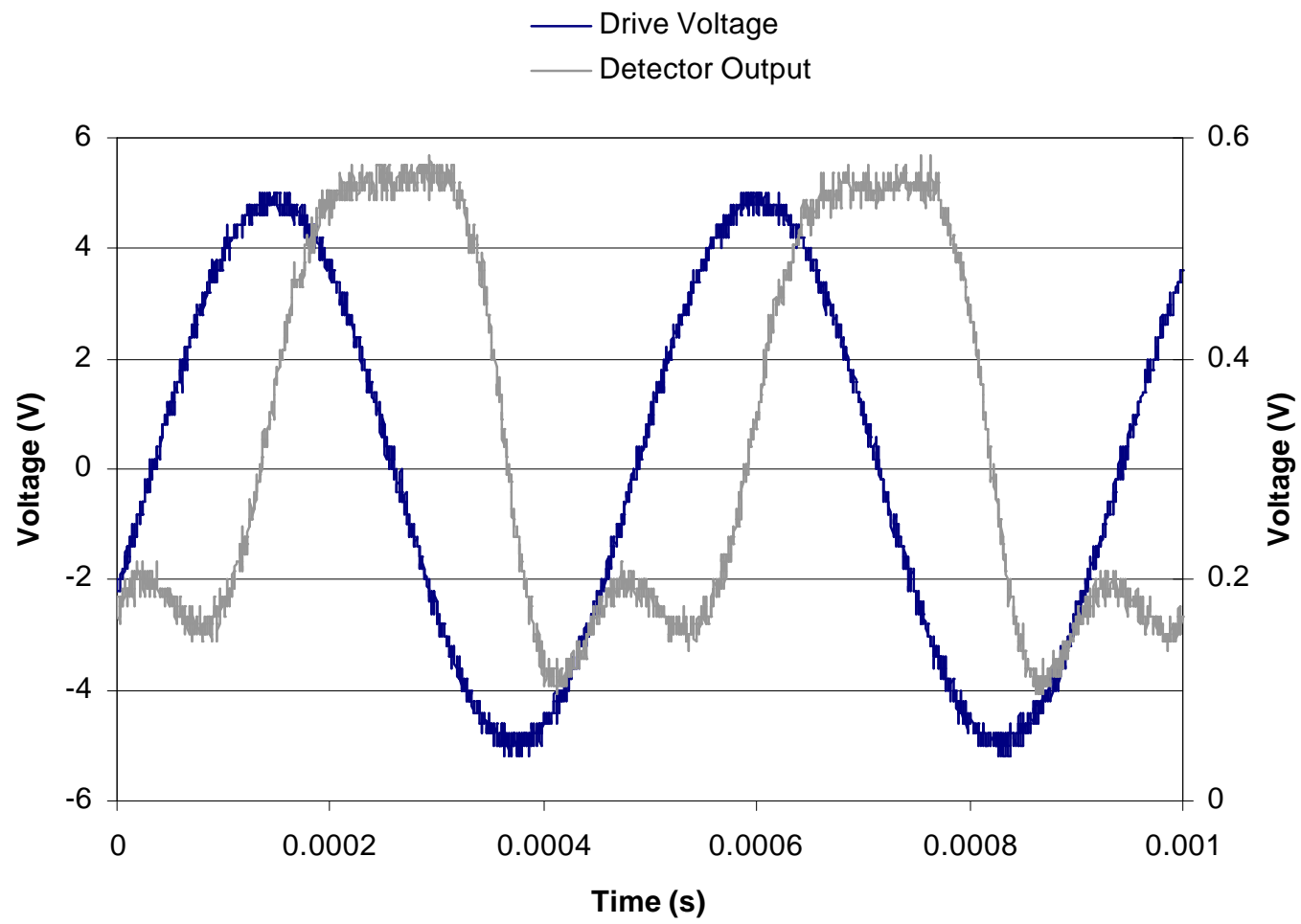

Figure 4.46: Output Comparison at $2.2 \mathrm{kHz}$

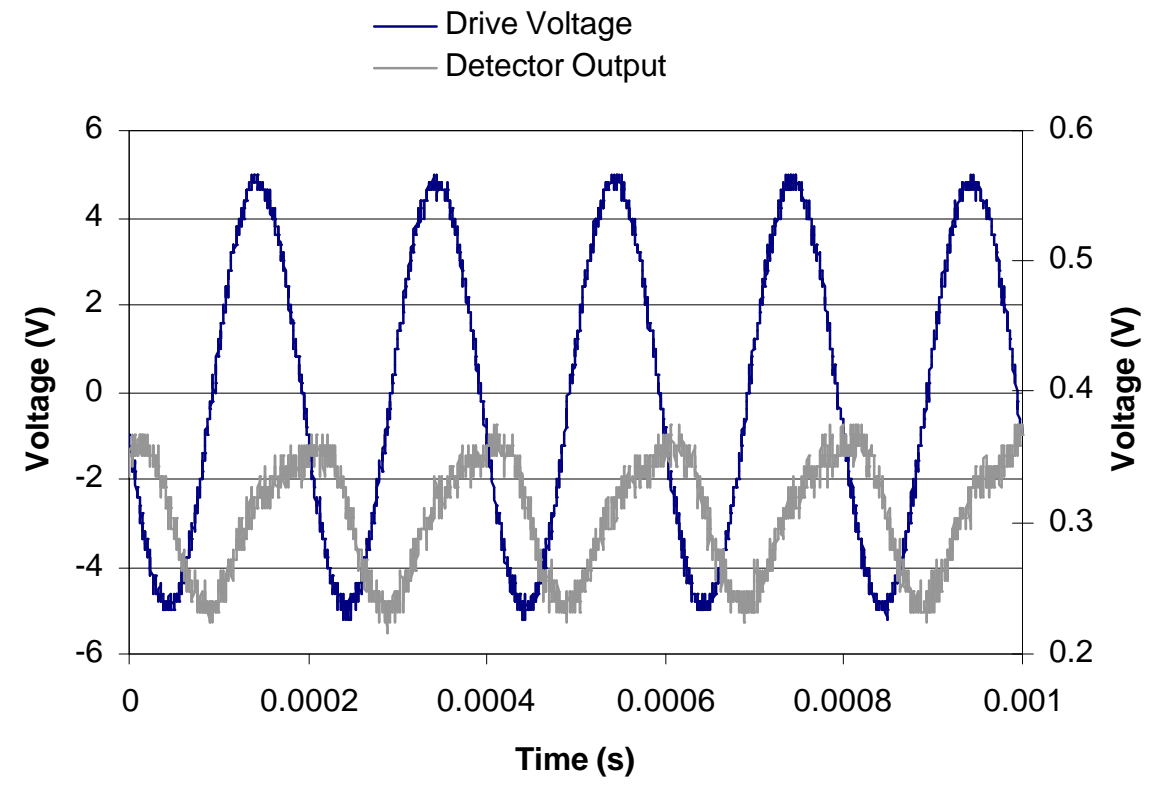

Figure 4.47: Output Comparison at $5 \mathrm{kHz}$ 


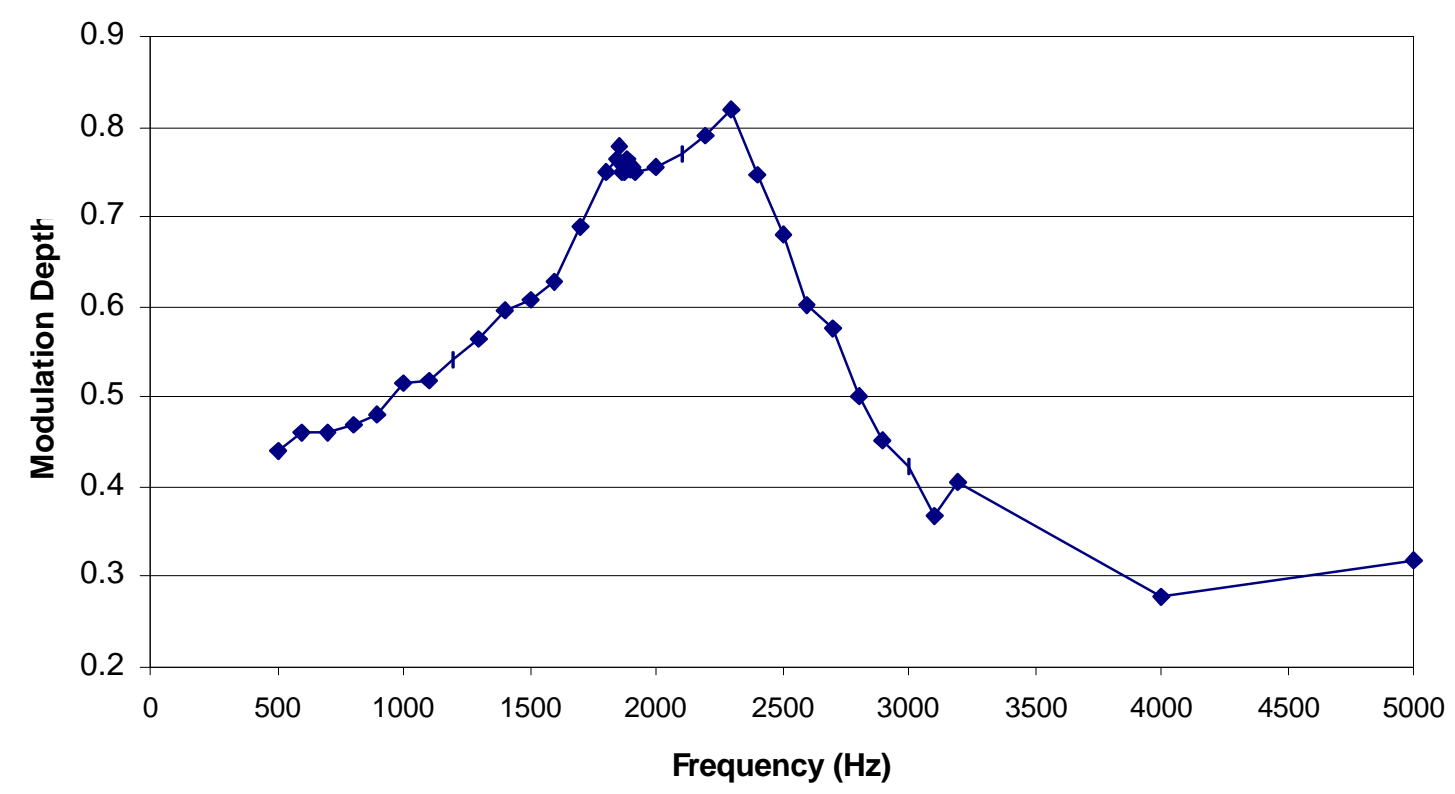

Figure 4.48: Modulation Depth: Frequency Variation

As the frequency of the drive voltage was increased, modulation depth also increased. This trend occurred until around $2.5 \mathrm{kHz}$ and then it started to decrease. Modulation depth should be greatest at resonance, but the resonant frequency of the device tested is $3.22 \mathrm{kHz}$ without a monolithic structure and $3.15 \mathrm{kHz}$ with the structure. The device fabrication process can produce angled sidewalls that may affect the width of the flexures. It can be observed from Equation 2.1-1 that there is a cubic dependence on flexure thickness, so varying this thickness will drastically affect the spring constant and resonant frequency of the flexures. The $30 \%$ difference in actual and theoretical resonant frequencies is most likely due to the non-ideal width of the flexures. At $2.2 \mathrm{kHz}$, the stage exhibited enough lateral movement that the monolithic structure moved completely over the spot, similar to results observed in the static displacement results. A phase shift in the optically monitored signal occurred as well, with the output becoming 90 degrees out of phase with the input at $5 \mathrm{kHz}$. This is most likely a result of the stage having more lateral 
displacement at higher frequencies. Changes in the DC level of the signal can be attributed to diffraction effects and drifting output power of the laser diode, which was current controlled not power controlled.

\subsubsection{Voltage Variation}

The device drive voltage was also varied in magnitude to observe how the optical output signal would change. Two sets of data were taken, one with the stator combs set at $\pm 10 \mathrm{~V}$ and the stage varied between 8 and $22 \mathrm{~V}$ peak-to-peak, and the other with the stator combs at $\pm 15 \mathrm{~V}$ and the stage varied between 10 and $30 \mathrm{~V}$ peak-to-peak. During both tests, frequency was kept constant at $500 \mathrm{~Hz}$. The laser diode was powered with 10 mA DC. Figures 4.49-4.51 show the results of selected values from the $\pm 15 \mathrm{~V}$ test. Both tests had similar trends in optical output results. Modulation depths for both sets of data are plotted in Figure 4.52.

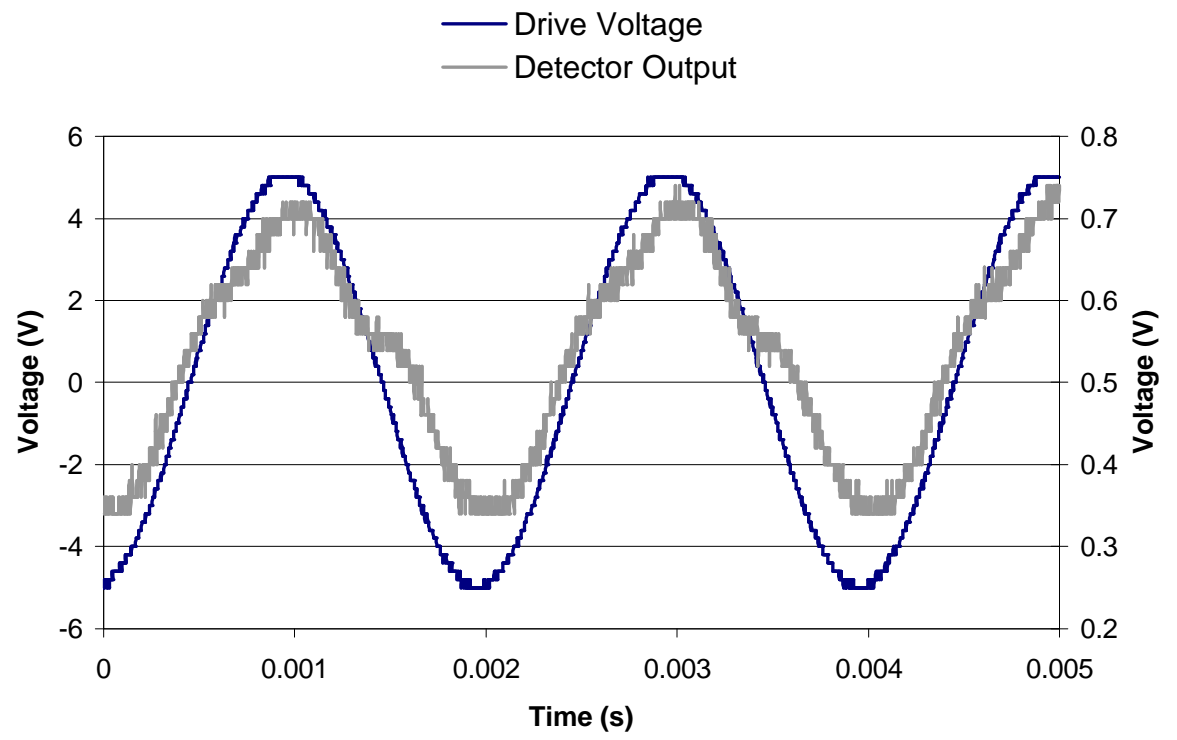

Figure 4.49: Output Comparison at $10 \mathrm{~V}_{\mathrm{pp}}$ 


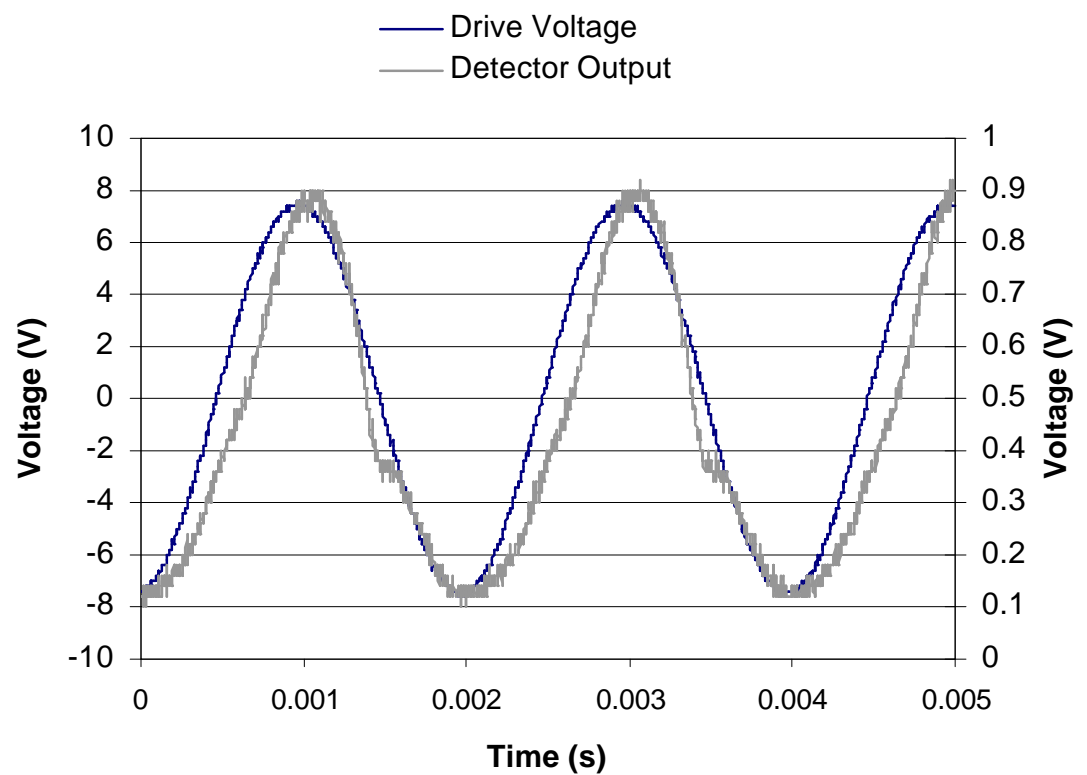

Figure 4.50: Output Comparison at $15 \mathrm{~V}_{\mathrm{pp}}$

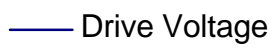

Detector Output

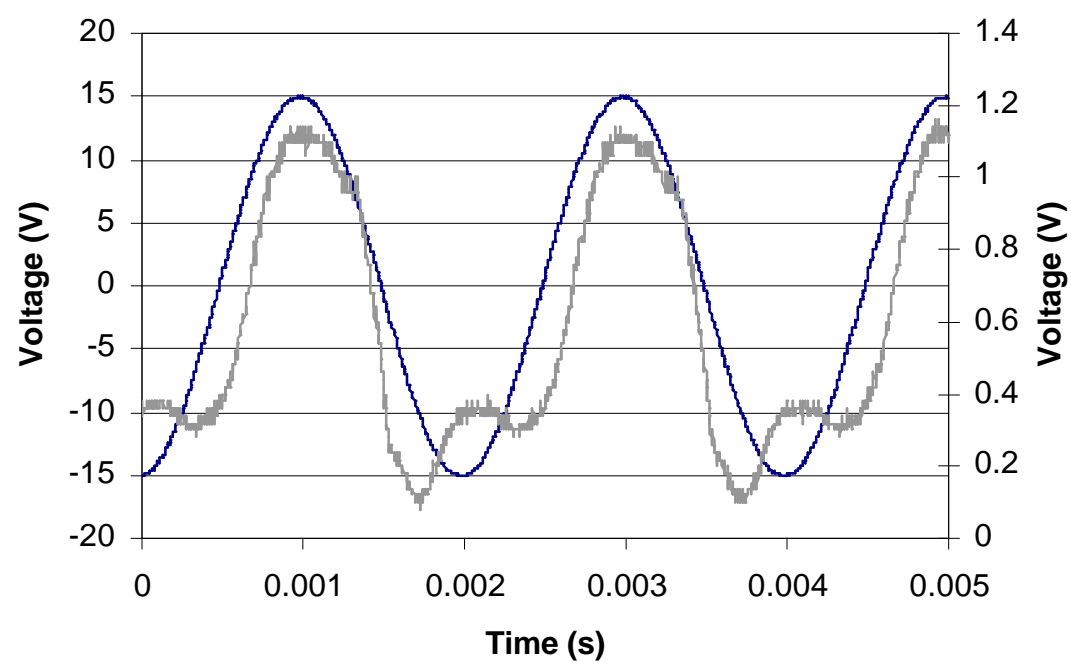

Figure 4.51: Output Comparison at $30 \mathrm{~V}_{\mathrm{pp}}$ 


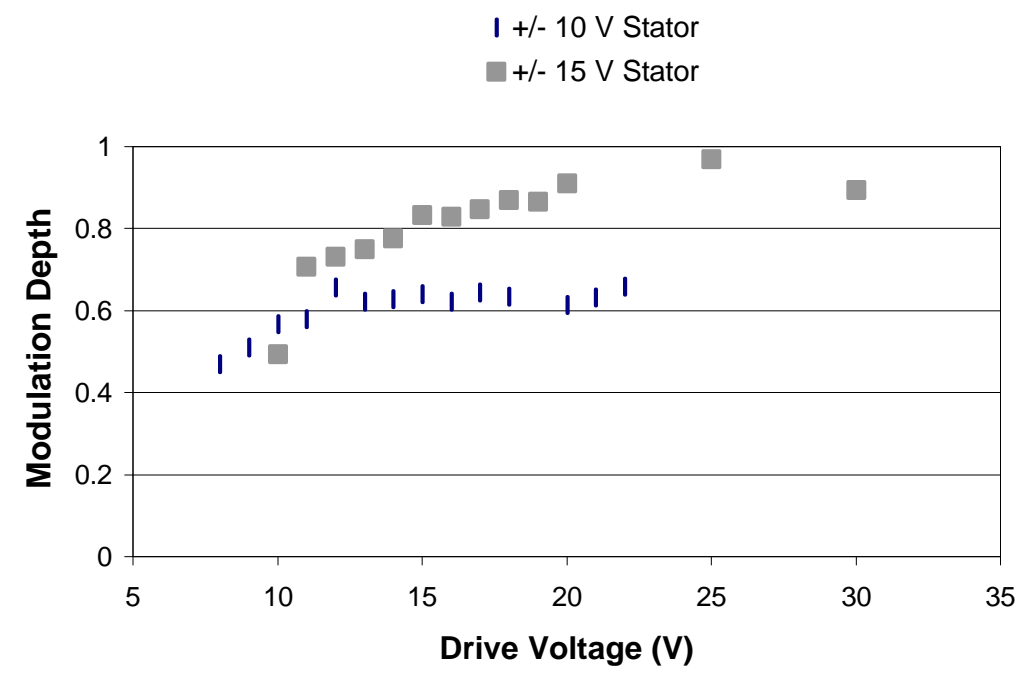

Figure 4.52: Modulation Depth: Voltage Variation

As drive voltage was increased, modulation depth increased as well. This is expected because the device stage travels farther at higher voltages. Effects in the monitoring seen at higher voltages are similar to those observed in both static displacement and frequency variation tests where the monolithic structure moves completely over the spot and allows light to pass through only POLY 1 on the other side. Irregularities in the waveforms at lower voltages are due to the spot not being initially positioned halfway underneath the monolithic structure as seen in Figure 4.40. The increase in the DC level of the signal is, again, a result of diffraction effects and input optical power drift. 


\section{Chapter 5}

\section{Loss Analysis and Conclusions}

The experimental system used to perform the through-wafer optical measurements, along with being very sensitive to outside vibrational interference, was also very lossy. This chapter will discuss the probable reasons for losses in intensity, and analyze sources of intensity loss in a preliminary integrated optical system that is the next logical step in the progression of this research. Conclusions drawn from experimental data and future directions of this research will also be presented.

\subsection{Experimental Setup Loss Analysis}

Even though many of the elements in the experimental setup had three directions of maneuverability, it was very difficult to manually achieve perfect alignment. Vibrational sensitivity also caused components to drift over time, making readjustment necessary. As with any other optical system, these misalignments were a major source of transmission losses.

Coupling losses from the laser diode to the first GRIN lens in the dual lens setup used can be as high as $4 \mathrm{~dB}$ if not positioned within $10 \mu \mathrm{m}$ of the lens' focal point [36]. 
The dual GRIN lens setup is very sensitive to position as well. A separation of $3 \mathrm{~cm}$ causes $2 \mathrm{~dB}$ of attenuation, and off-axis alignment by as little as $0.3 \mathrm{~mm}$ can cause $3 \mathrm{~dB}$ of loss to occur [36]. Light passing through the substrate experiences decreased transmission intensity as well. Measurements taken with no die present in the system gave $\sim 0.05 \mu \mathrm{W}$ of output power with an $8 \mu \mathrm{m}$ detector fiber. Measurements taken through the wafer over unpatterned regions gave $\sim 0.025 \mu \mathrm{W}$ of output power, a $50 \%$ decrease in intensity. All of these losses occur before the optical probe is effected by movement of the device stage.

After the translation stage causes a decrease in intensity, other factors cause loss as well. Since the detector fiber was not located at the focal point of the second lens, losses were caused from the angular divergence of the beam. At a distance of $1 \mathrm{~mm}$ away from the device surface, the beam diameter was $\sim 350 \mu \mathrm{m}$ (Figure 4.10 ). The $8 \mu \mathrm{m}$ detector fiber only covered $0.02 \%$ of the intensity distribution at this distance. Fiber coupling to the InGaAs detector is another area of loss as well.

\subsection{Loss Analysis of Proposed Integrated Through- Wafer Optical Monitoring System}

The setup shown in Figure 5.1 is an integrated through-wafer monitoring system similar to the experimental setup. It uses integrated Fresnel lenses and plane waveguides to direct light from an input to the device plane and out to a detector. 


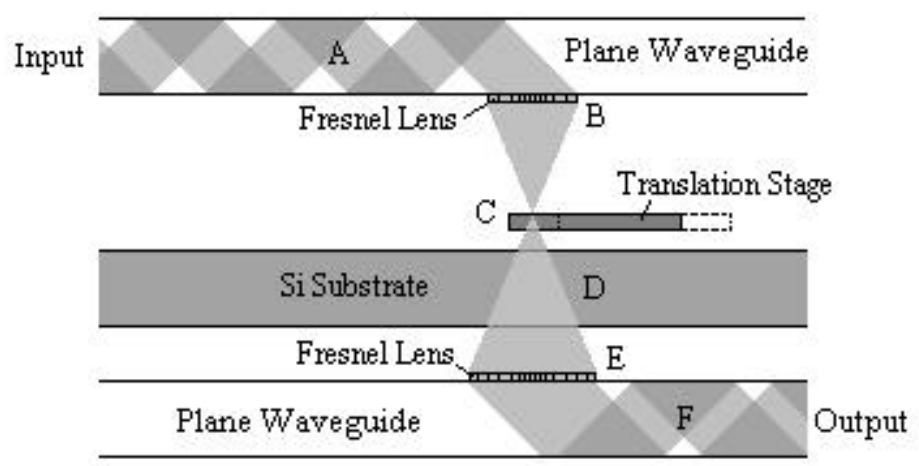

Figure 5.1: Loss Areas in an Integrated Through-Wafer Optical Monitoring System

Due to its compact size and improved alignment, this system will not suffer from the high vibrational sensitivity and off-axis alignment losses that were experienced in the experimental setup. However, losses will occur elsewhere. The losses in the input and output waveguides (A and F) will be negligible. However, coupling to and from them is done using integrated Fresnel lenses (B and D), which have coupling efficiencies of 40$98 \%$, depending on the number of phase levels [29]. It has been shown by the experiments in this research that the moving stage (C) can cause transmission intensities to drop from 45 to $85 \%$, and that the substrate causes a $50 \%$ decrease. The total transmission percentage of this system is given in table 5.1 for both binary and multilevel Fresnel lenses with and without stage interruption.

Table 5.1: Integrated Optical Monitoring System Transmission Percentage

\begin{tabular}{|c|c|c|c|c|}
\hline & \multicolumn{2}{|c|}{ Stage Interruption } & \multicolumn{2}{|c|}{ Uninterrupted } \\
\hline & Binary & Multi-level & Binary & multi-level \\
\hline $\begin{array}{c}\text { Percent } \\
\text { Transmission }\end{array}$ & $4-1 \%$ & $26-7 \%$ & $8 \%$ & $48 \%$ \\
\hline
\end{tabular}




\subsection{Conclusions and Future Work}

The experimental results shown in Chapter 4 demonstrate the feasibility of a through-wafer optical method of monitoring MEMS microstructures. Image capture experiments gave assurance that a decrease in intensity of the through-wafer probe as it passed under the features of the device stage would be enough to give a strong output signal. Through-wafer power measurements show that the percent change in transmission intensity is $75-20 \%$ for a $47 \mu \mathrm{m}$ detector fiber, and $66-2 \%$ for an $8 \mu \mathrm{m}$ detector. These measurements also show that diffraction effects caused by device topology can enhance the range of the output signal.

Output waveforms of the optically monitored continuously moving stage vary with the location of the through-wafer probe, as well as the input voltage parameters of the device. These changes are a result of the features of the MEMS device. Changing the position of the probe with respect to the monolithic structure affects the phase of the output signal. Increasing the frequency of the drive voltage causes an increase in signal modulation depth. This modulation depth should be greatest at resonance, but is effected by the monolithic structure to cause a lower value than theoretically expected. The shape of the waveform is affected by the POLY 2 strip on the device stage due to increased lateral displacement. Modulation depth decreases as the frequency is increased past the point of resonance. Increasing the magnitude of the sinusoidal drive voltage also increases modulation depth, and again, increased lateral displacement causes device features to affect the shape of the output waveform.

Losses in the system due to component misalignment are a source of decreased signal intensity. A more stable system with a finer degree of manipulation should be 
devised in order to reduce these losses and improve data gathering capabilities. Losses in an integrated system primarily stem from through-wafer reflection losses and low coupling efficiencies of binary integrated Fresnel lenses. Anti-reflection coatings on the substrate and multi-level Fresnel lenses could increase system transmission characteristics. The next step of this research is to co-integrate the optics used to deliver the optical signal with MEMS devices to create a self-contained monitoring system. New device designs more suitable for through-wafer probing should also be explored. Ultimately, this monitoring system could be combined with integrated sources, detectors and control circuitry to create a feedback and control system that will be in place for the lifetime of the device. 
Appendix A

MATLAB MEMS Analysis Programs 


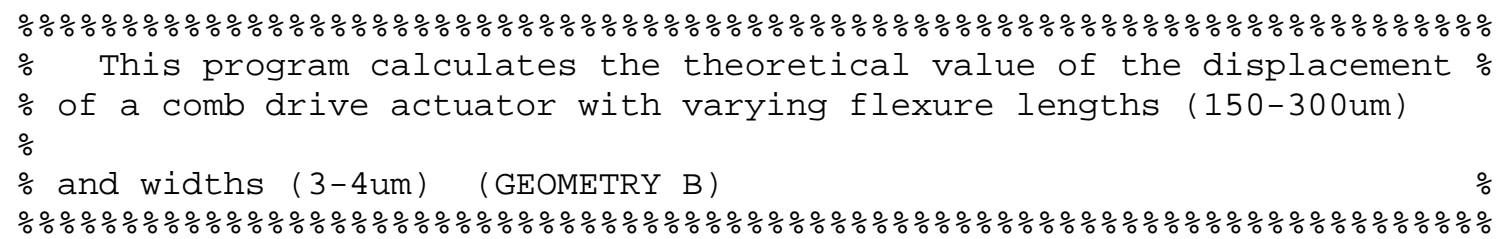


\% voltage variation loop for 3 um

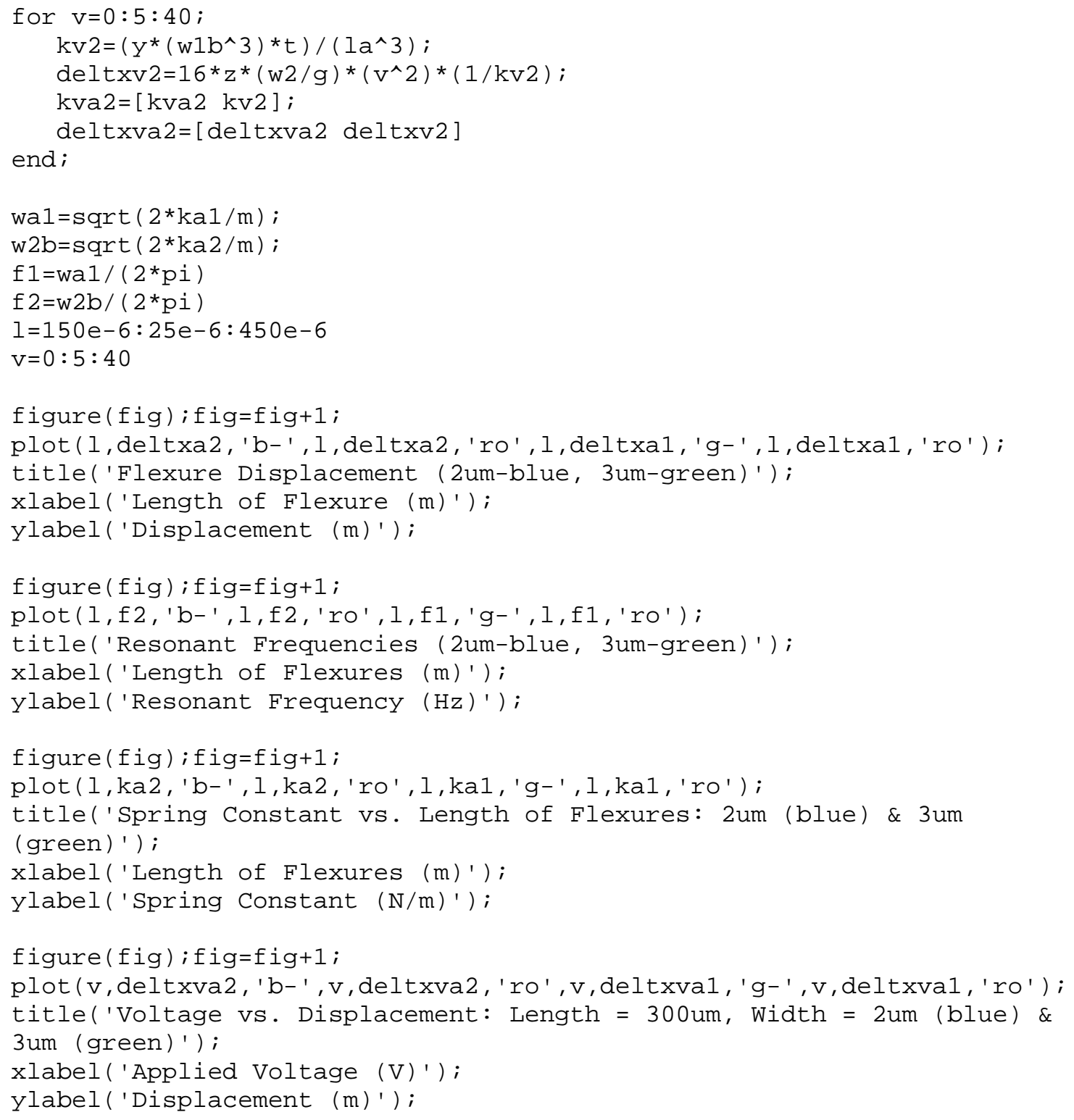




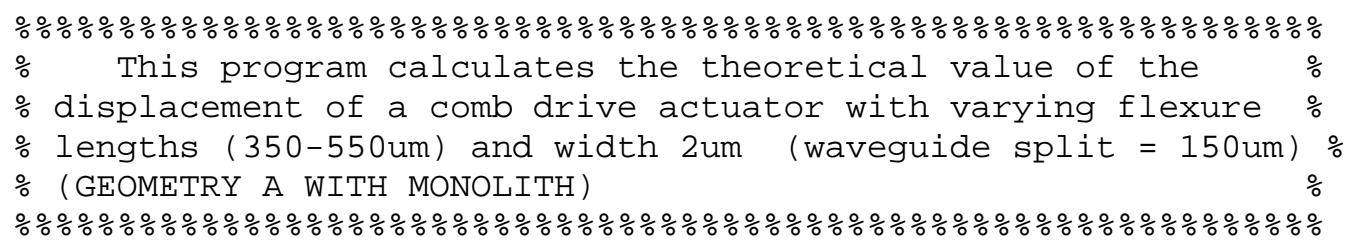




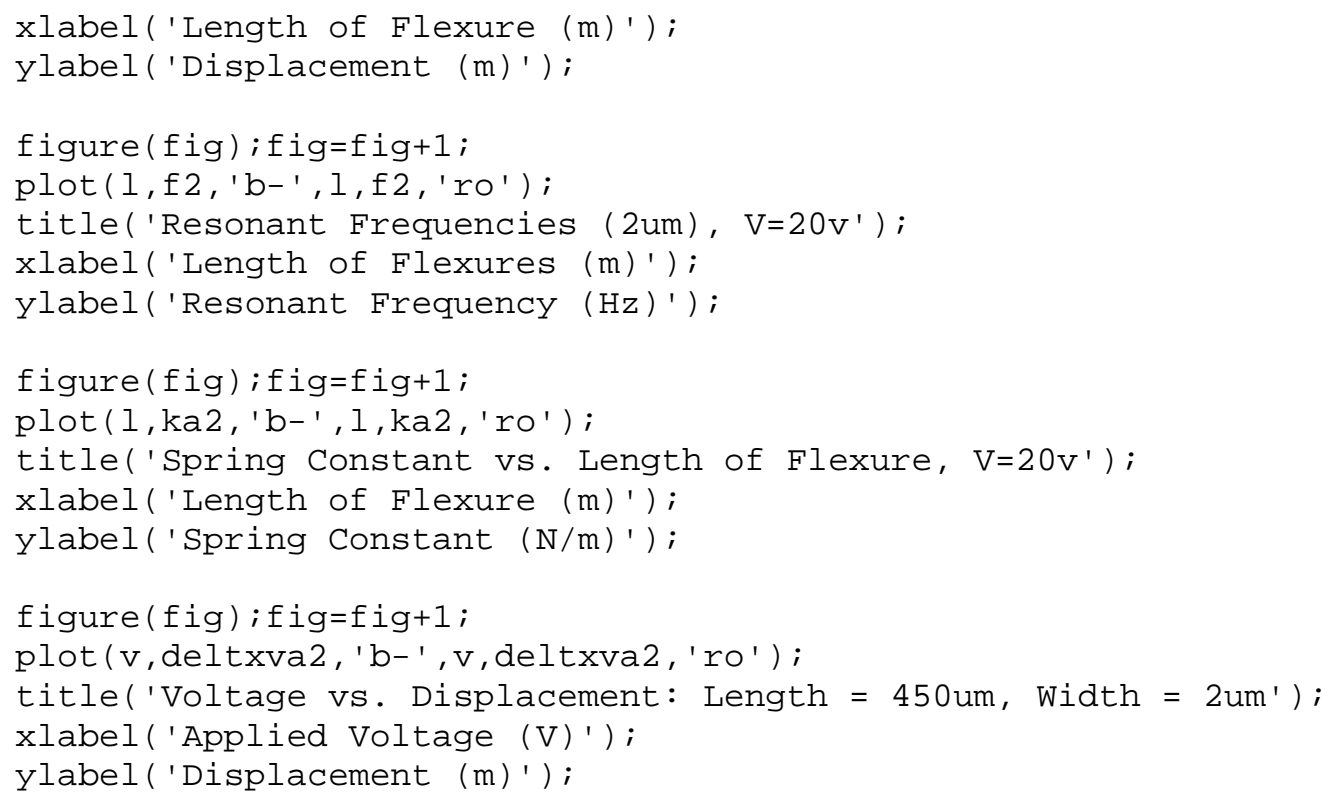




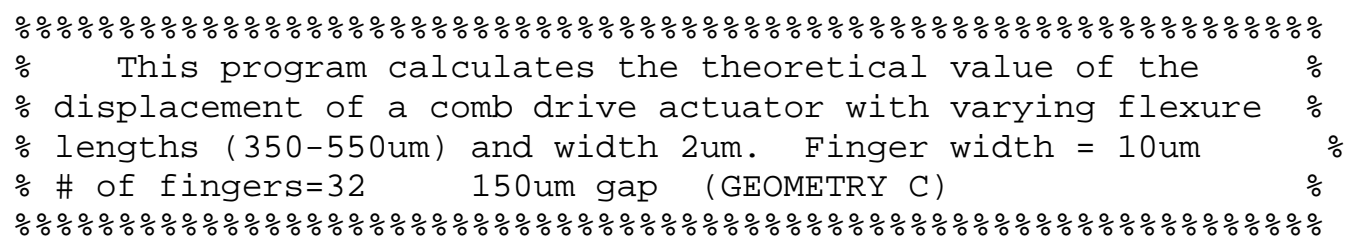




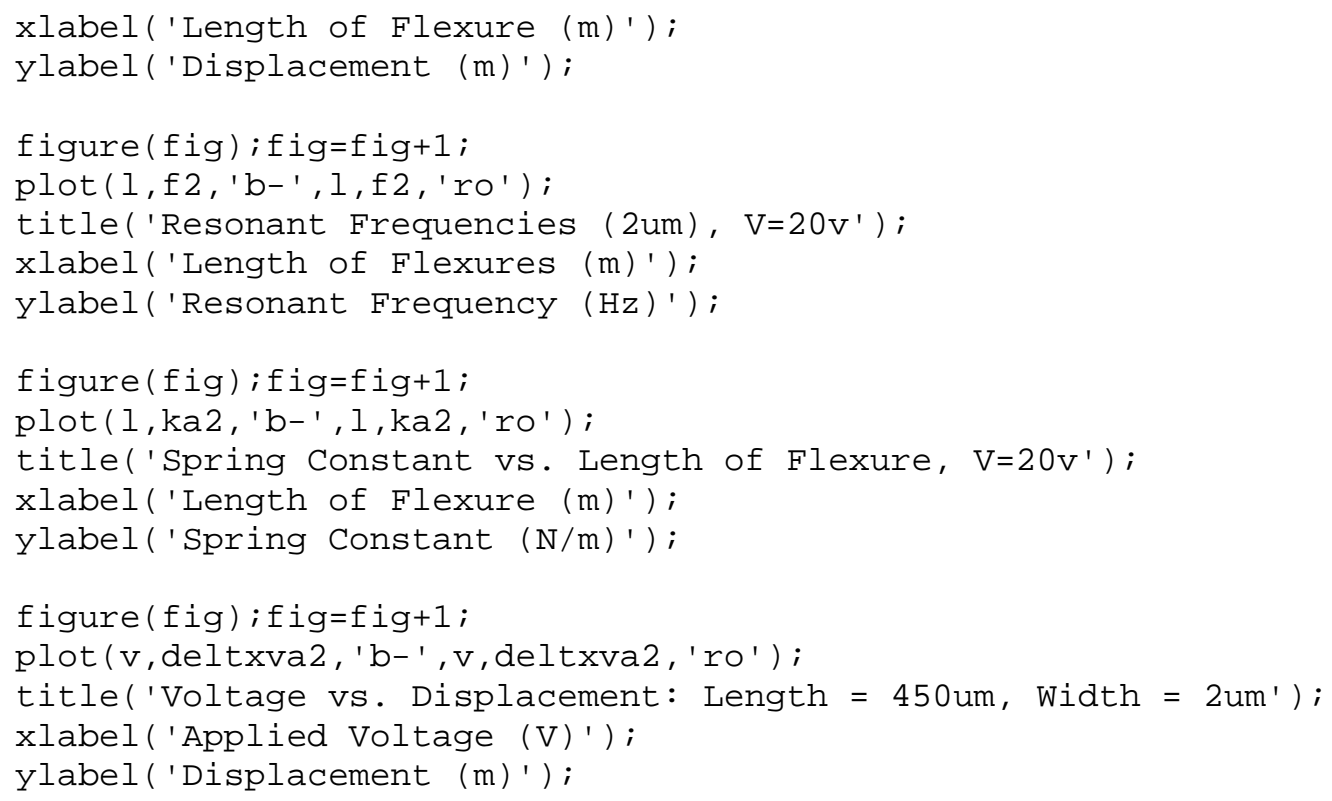




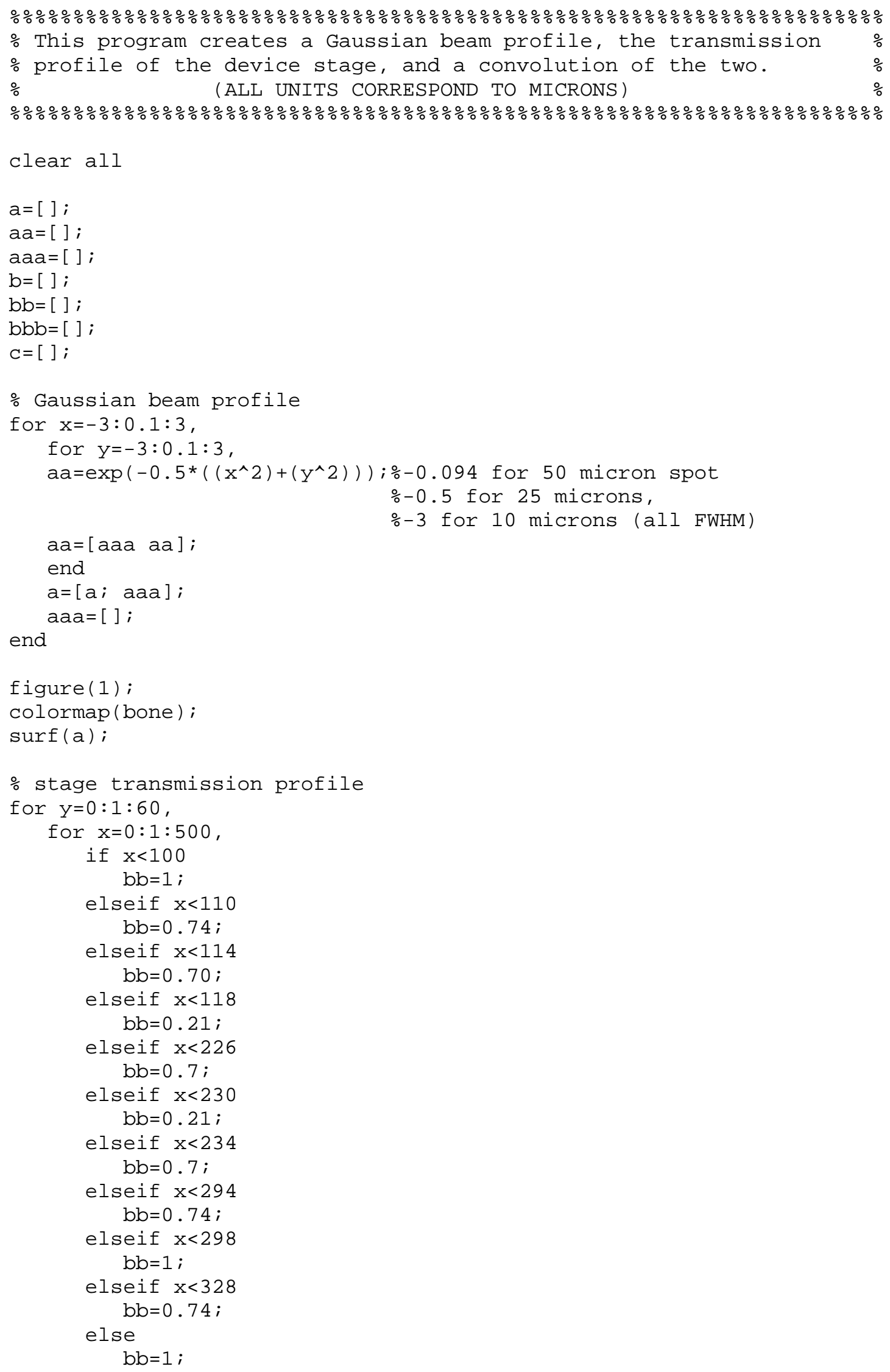




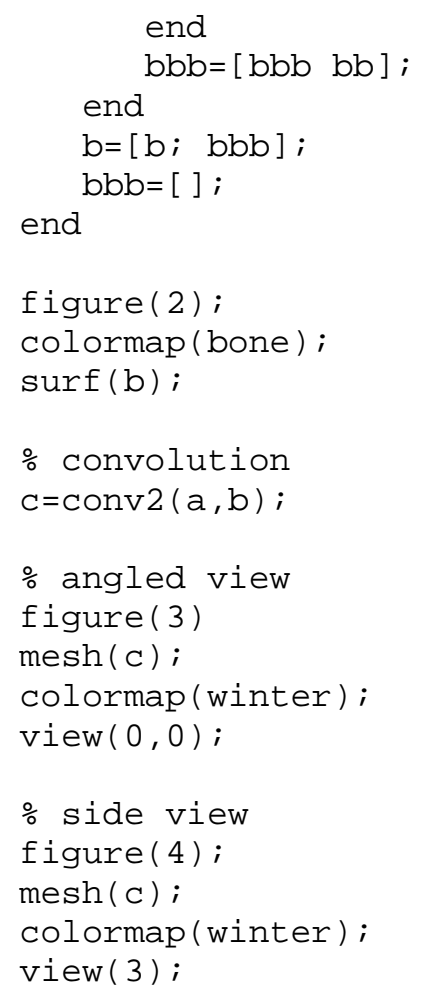




\section{Appendix B}

\section{Microelectronics Center of North Carolina (MCNC) Multi-User MEMS Processing Service (MUMPS) and Film Parameters}

All devices used in this research were fabricated at MCNC in their MUMPS

facility. Their fabrication process uses multilevel photolithographic masking and deposition of polysilicon by low-pressure chemical vapor deposition (LPCVD) combined with LPCVD deposited oxide layers to create releasable microstructures. This process is outlined in great detail at their web site [42]. The following table shows the film parameters for MUMPS 20, the device fabrication run of the devices used. 
Table B.1 Film Parameters for MUMPS 20 [42]

\begin{tabular}{|c||c||c||c|c|c||}
\hline Film & $\begin{array}{c}\text { Thickness } \\
(\mathbf{A})\end{array}$ & $\begin{array}{c}\text { Std. Dev. } \\
(\mathbf{A})\end{array}$ & $\begin{array}{c}\text { Sheet Resist. } \\
(\mathbf{o h m} / \mathbf{s q .})\end{array}$ & $\begin{array}{c}\text { Resistivity } \\
(\mathbf{o h m}-\mathbf{c m})\end{array}$ & $\begin{array}{c}\text { Stress } \\
\text { (Mpa) }\end{array}$ \\
\hline Nitride & 5990 & 165 & - & - & $132(\mathrm{~T})$ \\
\hline \hline POLY 0 & 5001 & 12 & 25.8 & $1.2 \mathrm{E}-3$ & $24(\mathrm{C})$ \\
\hline \hline Oxide 1 & 19794 & 533 & - & - & - \\
\hline \hline Dimple & 7115 & 342 & - & - & - \\
\hline \hline POLY1 & 19428 & 45 & 10.8 & $2.0 \mathrm{E}-3$ & $8(\mathrm{C})$ \\
\hline \hline Oxide 2 & 7440 & 178 & - & - & - \\
\hline \hline POLY 2 & 14837 & 68 & 20.0 & $2.9 \mathrm{E}-3$ & $9(\mathrm{C})$ \\
\hline \hline $\begin{array}{c}\text { Metal } \\
(\mathrm{Cr} / \mathrm{Au})\end{array}$ & 5250 & - & 0.0506 & $2.6 \mathrm{E}-6$ & $95(\mathrm{~T})$ \\
\hline
\end{tabular}

Figures B.1 and B.2 show polysilicon stress and resistivity trends for MUMPS runs 5-29.

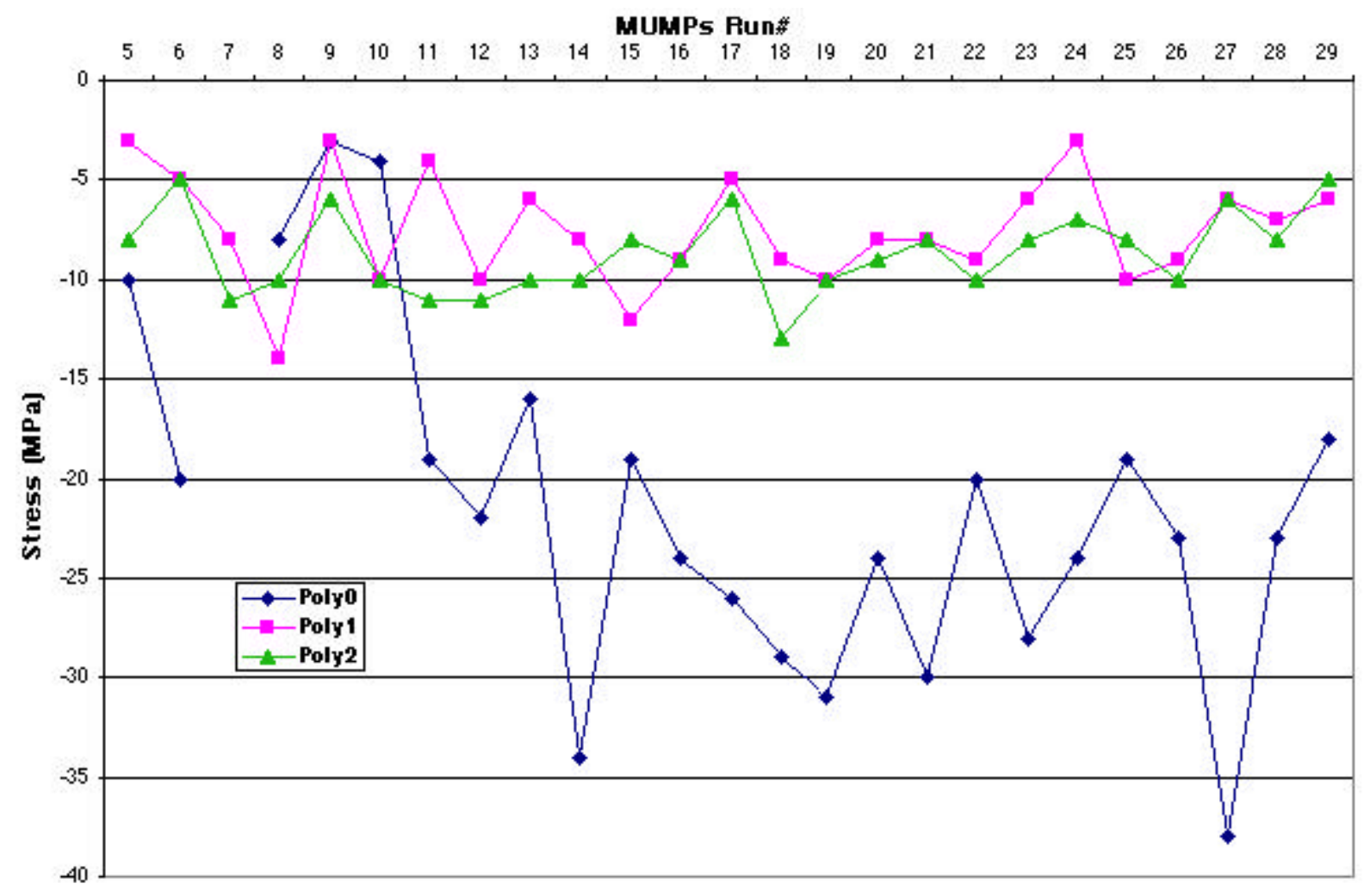

Figure B.1: Polysilicon Stress Trends [42] 


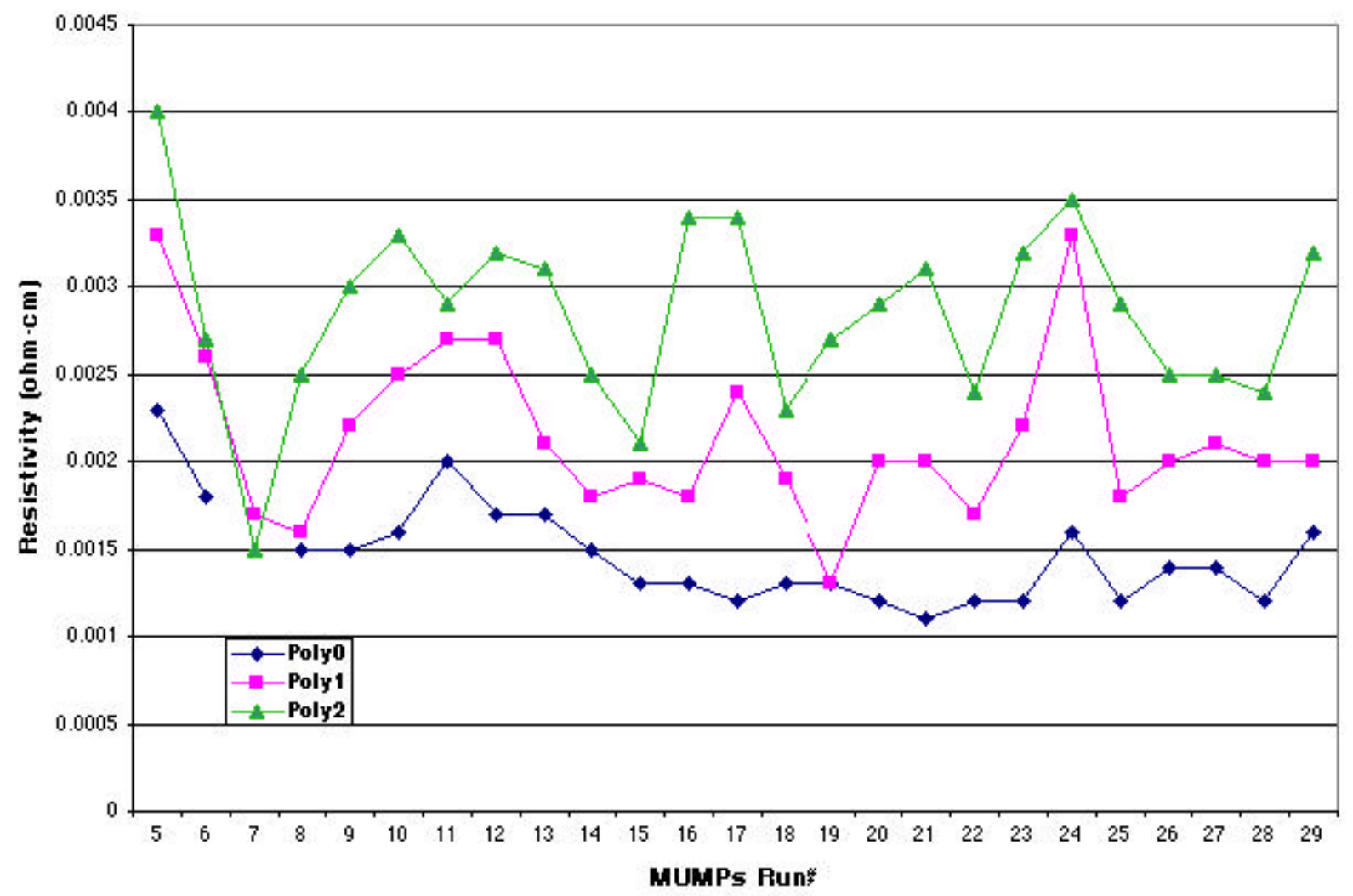

Figure B.2: Polysilicon Resistivity Trends [43] 


\section{Bibliography}

[1] F. Merat and M. Mehregany, "Integrated micro-opto-electro-mechanical systems," Proc. SPIE, vol. 2383, pp. 88-98.

[2] P. R. Nelson, P. B. Chu, and K. S. J. Pister, "Optical methods for characterization of MEMS device motion," Proc. SPIE, vol. 2640, pp. 53-57.

[3] J. S. Burdess, A.J. Harris, D. Wood, R. J. Pitcher, and D. Glennie, "A System for the Dynamic Characterization of Microstructures," Journal of Microelectromechanical Systems, vol. 6, no. 4, pp. 322-328, Dec. 1997.

[4] W. D. Cowan, V. M. Bright, and G. C. Dalton, "Measuring frequency response of surface-micromachined resonators," Proc. SPIE, vol. 3225, pp. 32-43.

[5] G. K. Fedder and R. T. Howe, "Multimode Digital Control of a Suspended Polysilicon Microstructure," Journal of Microelectromechanical Systems, vol. 5, no. 4, pp. 283-297, Dec. 1996.

[6] H. H. Pham and A. Nathan, "Compact MEMS-SPICE Modeling," Sensors and Displays, vol. 10, no. 2, pp. 63-75, 1998.

[7] T. Olbrich, A. Richardson, W. Vermeiren, and B. Straube, "Integrating testability into microsystems," Microsystem Technologies, pp. 72-79, 1997.

[8] P. May, S. T. Wilkinson, et. al., "Design Issues for Through-Wafer Optoelectronic Interconnects," Proc. IEEE, pp. 8-15, 1995.

[9] M. E. Motamedi, M. C. Wu, and K. S. Pister, "Micro-Opto-Electro-Mechanical Devices and On-Chip Optical Processing," SPIE - Optical Engineering, vol. 36, no.5, pp. 1282-1297, May 1997.

[10] M. C. Roggeman, V. M. Bright, et. al., "Use of Micro-Mechanical Deformable Mirrors to Control Abberations in Optical Systems: Theoretical and Experimental Results," SPIE - Optical Engineering, vol. 36, no.5, pp. 1326-1338, May 1997. 
[11] T. G. Bifano, R. K. Mail, et. al., "Continuous-membrane surface-micromachined silicone deformable mirror," SPIE - Optical Engineering, vol. 36, no.5, pp. 13541360, May 1997.

[12] J. J. Sniesowski and E.J. Garcia, "Microfabricated Actuators and Their Application to Optics," Proc. SPIE, vol. 2383, pp. 46-64.

[13] S. K. Tewksbury and L. A. Hornak, "Multichip Modules: A Platform for Optical Interconnects Within Microelectronic Systems," Optoelectronics-Devices and Technologies, vol. 9, no. 1, pp. 55-80, March 1994.

[14] S. Koh, D. J. Sadler, and C. H. Ahn, "Optoelectronic Multichip Modules for High Speed Computer Systems and Communication Networks," SPIE - Optical Engineering, vol. 36, no.5, pp. 1319-1325, May 1997.

[15] D. L. Dickensheets, "Imaging performance of off-axis planar diffractive lenses," Journal of the Optical Society of America A, vol. 13, no. 9, pp. 1849-1858, Sept. 1996.

[16] D. V. Svistunov, "Developed passive components of planar optical schemes," Optical Engineering, vol. 33, no. 10, pp. 3171-3177, Oct. 1994.

[17] K. S. Brown, B. J. Taylor, L. A Hornak, and T. W. Weidman, "Characteristics of Poly(Phenylsilsesquioxane) Thin-Film Planar Optical Waveguides," IEE Photonics Technology Letters, vol. 9, no. 6, June 1997.

[18] K. Benaissa and A. Nathan, "IC Compatible Optomechanical Pressure Sensor Using Mach-Zender Interferometry," IEEE Transactions on Electron Devices, vol. 43, no. 9, pp. 1571-1582, September 1996.

[19] D. Hofstetter, H. P. Zappe, and R. Dändliker, "Optical Displacement Measurement with GaAs/AlGaAs-Based Monolithically Integrated Michelson Interferometers," Journal of Lightwave Technology, vol. 15, no. 4, pp. 663-669, April 1997.

[20] H. Helmers, P. Benech, and R. Rimet, "Integrated Optical Components Employing Slab Waveguides for Sensor Applications," IEE Photonics Technology Letters, vol. 8, no. 1, pp. 81-83, Jan. 1996.

[21] L. A. Hornak and S. K. Tewksbury, "ON THE Feasibility of Through-Wafer Optical Interconnects for Hybrid Wafer-Scale Integrated Architectures," IEEE Transactions on Electron Devices, vol. ED-34, no. 7, pp. 1557-1563, July 1987. 
[22] S. S. Lee, L. Y. Lin, K. S. J. Pister, et. al., "Passively Aligned Hybrid Integration of 8x1 Micromachined Micro-Fresnel Lens Arrays and 8x1 Vertical-Cavity SurfaceEmitting Laser Arrays for Free-Space Optical Interconnect," IEEE Photonics Technology Letters, vol. 7, no. 9, pp. 1031-1033, Sept. 1995.

[23] N. M. Jokerst, C. Camperi-Ginestet, B. Buchanan, et. al., "Communication Through Stacked Silicon Circuitry Using Integrated Thin Film InP-Based Emitters and Detectors," IEEE Photonics Technology Letters, vol. 7, no. 9, pp. 1028-1030, Sept. 1995.

[24] D. S. Wills, W. S. Lacey, C. Camperi-Ginestet, et. al., "A Three-Dimensional High-Throughput Architecture Using Through-Wafer Optical Interconnect," Journal of Lightwave Technology, vol. 13, no. 6, pp. 1085-1091, June 1995.

[25] L. A. Hornak, "Fresnel phase plate lenses for through-wafer optical interconnections," Applied Optics, vol. 26, no. 17, pp. 3649-3654, Sept.1987.

[26] E. Pawlowski, "Integrated planar Fresnel zone lenses for beam forming and coupling," Proc. SPIE, vol. 2213, pp. 89-97.

[27] S. Ogata and Y. Ito, "Laser-diode collimating light sources using micro-Fresnel lenses," Optical Engineering, vol. 33, no. 11, pp. 3656-3661, Nov. 1994.

[28] E. A. Watson, "Analysis of beam steering with decentered microlens arrays," Optical Engineering, vol. 32, no. 11, pp. 2665-2670, Nov. 1993.

[29] M. Ferstl and A-M. Frisch, "Static and dynamic Fresnel zone lenses for optical interconnections," Journal of Modern Optics, vol. 43, no. 7, pp. 1451-1462, 1996.

[30] H. Zarschizky, A. Stemer, F. Mayerhofer, et. al., "Binary and multilevel diffractive lenses with submicrometer feature sizes," Optical Engineering, vol. 33, no. 11, pp. 3527-3536, Nov. 1994.

[31] G. Fedder, Microelectromechanical Design, web page, http://www.ece.cmu.edu/afs/ece.cmu...usr/fedder/www/flexures.

[32] H. J. Lee, M. Tachiki, C. Liu, K. S. J. Pister, UCLA Automated MEMS Design and Analysis Tools - The Design of Resonant Microstructures, University of California, Los Angeles Summer Research Program Final Report, 1994.

[33] E. Hecht, Optics, Addison-Wesley Publishing Co., Redding, Mass., 1987.

[34] M. Born and E. Wolf, Principles of Optics, Cambridge University Press, Cambridge, UK, 1998. 
[35] B. E. A. Saleh and M. C. Teich, Fundamentals of Photonics, Wiley-Interscience, New York, 1991.

[36] NSG America, Inc., SELFOC Handbook, 1985.

[37] G. Hatakoshi and K. Goto, "Grating lenses for the semiconductor wavelength," Applied Optics, vol. 24, no. 24, pp. 4307-4311, Dec. 1985.

[38] J. C. Palais, Fiber Optic Communications, Prentice Hall, Englewood Cliffs, New Jersey, 1992.

[39] Dr. Halliburton and K. Stevens, Physics Dept. West Virginia University.

[40] L. A. Hornak, "Through-Wafer Optical Interconnection Coupling Characteristics," Electronics Letters, vol. 24, no. 11, pp. 714-715, May 1998.

[41] L. A. Hornak, Optical Interconnections and Wafer-Level Systems, Electrical Engineering Ph.D. Dissertation, Rutgers University, May 1991.

[42] D. A. Koester, MUMPs Design Handbook, web page, http://mems.mcnc.org/mumps.html. 